$\sum_{n}^{\infty}$

co

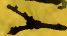



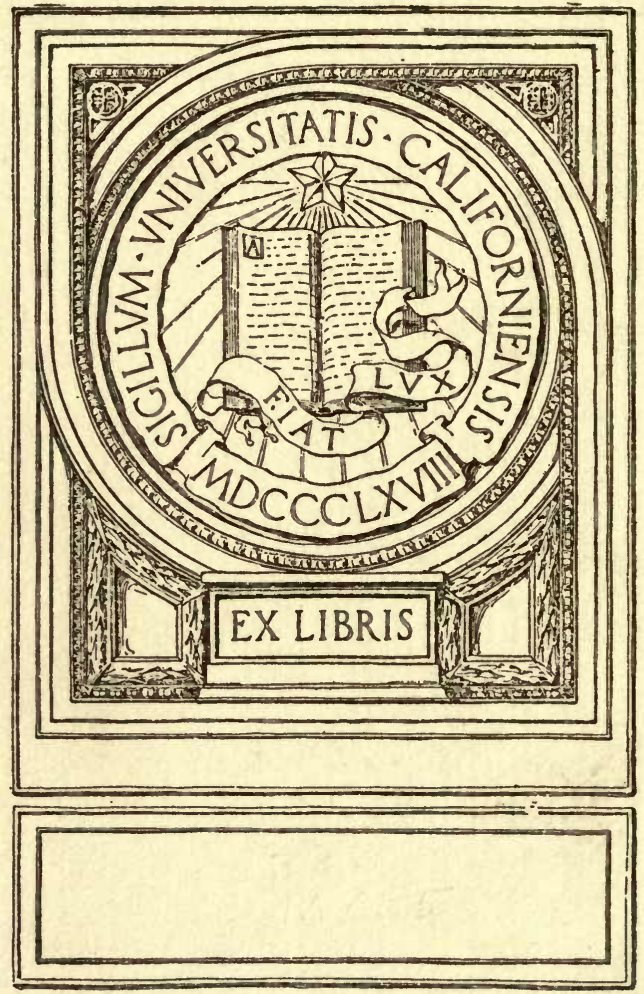





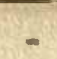


THE LIMITATIONS OF SCIENCE 



\title{
THE LIMITATIONS OF SCIENCE
}

\author{
BY

\section{LOUIS TRENCHARD MORE, PH.D. \\ Professor in the University of Cincinnati} \\ Professor in the Universily of Cincinnali
}

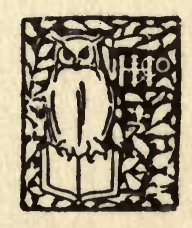

NEW YORK

HENRY HOLT AND COMPANY

1915 

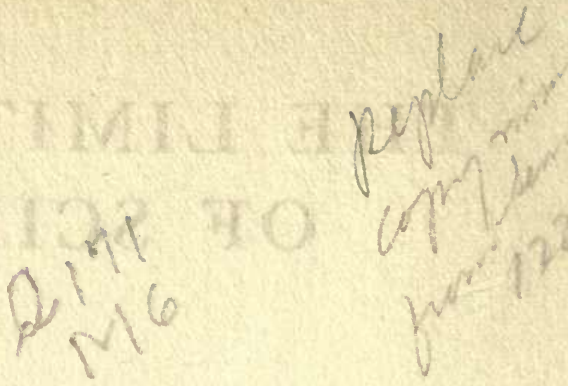

\section{COPYRIGHT, 1915, \\ BY \\ LOUIS TRENCHARD MORE}

Published April, I915
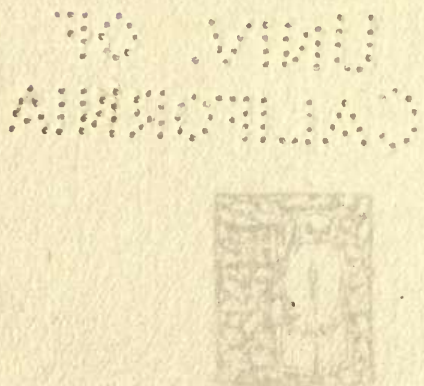

THE QUiNn a boden CO. PREB RAHWAY, N. d. 
Hypotheses non fingo. Quicquid enim ex phænomenis non deducitur, hypothesis vocanda est; et hypotheses seu metaphysicæ, seu physicæ, seu qualitatum occultarum, seu mechanicæ, in philosophia experimentali locum non habent. In hac philosophia propositiones deducuntur ex phænomenis, et redduntur generales per inductionem.-NEWTON.

\section{2}





\section{PREFACE}

THE separate chapters of this book have already been published as essays in periodicals. The first, second, third, and seventh appeared in The Hibbert Journal; the fourth, in The Philosophical Magazine and in the University of Cincinnati Studies; the fifth, in The Monist; and the sixth, in The Unpopular Review. But they have been rewritten and enlarged so as to form chapters for a connected discussion of the Limitations of Science. This was not a difficult task since I have had this purpose in mind from the beginning.

Cincinnati,

December 2, 1914. 



\section{CONTENTS}

CHAPTER

I Science as a Symbol and a Law . . I

II The Metaphysical Tendencies of Modern Physics . . . . . . . . 32

III Descartes and His Influence on Recent SCIENCE • . . . . . . 69

IV The Scientific Method . . . . 107

V The Classical and the New Mechanics 142

VI Skepticism and Idolatry in Science • • 187

VII Science as the Arbiter of Ethics • . 212 INDEX • • • . . . . . 263 



\title{
THE LIMITATIONS OF SCIENCE
}

\author{
CHAPTER I \\ SCIENCE AS A SYMBOL AND A LAW
}

Les théories contemporaines sont sur ce point d'accord avec l'histoire; elles consacrent la prépondérance dans le domaine scientifique, de l'hypothèse atomistique.-HANNEQuin.

IT has grown to be an axiom in modern thought, that the hope of discovering the laws of nature and our relation to them by metaphysical reasoning is impossible. The term metaphysical reasoning will be used consistently to mean the method introduced by the Greek philosophers who, however they might differ in minor matters, were pretty well agreed in looking upon what we call nature as something which could be investigated subjectively; that is, things are as we think them to be. It is thus directly contrary to the physical method, which maintains natural phenomena and laws to be entirely objective and independent of our thought; according to this method no theory or fact can be established unless it is completely verified by experimental tests.

So little in the long years since Plato and Aristotle has been done by the metaphysical philosophers to add 
to our positive knowledge, that they themselves are abandoning their former methods for that of the psychologist, who studies even the faculties and the emotions of the mind objectively by means of the physical or experimental method. Many will frankly admit that metaphysical studies are chiefly valuable now as a history of the development of thought, and agree with Renan that "Science, and science alone, can give to humanity what it most craves, a symbol and a law." If this be really the case, if the attainment of our desire for an accurate and real knowledge of our environment, its phenomena and the causes of actions, rests with science alone, then it becomes necessary to consider whether this hope also must prove to be fallacious. Will the results of experiment made objectively, which must however be interpreted subjectively, fail in their turn as criteria of truth?

Of the various sciences, physics offers probably the best means of attacking this problem, for it lies between the concrete classifications of the natural sciences, such as chemistry and biology, and the abstract theories of pure mathematics. Physics, on the one hand, is less disturbed by the multitude of details which often, in the natural sciences, prevent the grasping of a central idea; while, on the other hand, it is more circumscribed than mathematics by the necessity of constant comparison with concrete phenomena, and so avoids the danger of confounding speculation and 


\section{SCIENCE AS A SYMBOL AND A LAW}

reality. Thus the methods of physics have, to a degree, become the model which the other sciences seek to follow, a logical mathematical theory based on, and corrected by, experimental observation. Moreover, this science presents a longer and more consecutive history than most of the others.

It is also noticeable that physics treats of problems similar to those of metaphysics. During the sixteenth, seventeenth, and eighteenth centuries philosophy and physics were closely united, and were largely dominated by the deductive method, which was introduced by Descartes and which led to persistent attempts to explain scientific laws by metaphysical causes. It was then that the two great metaphysical ideas, of the continuity of matter with its identification with space, and of matter as a form of indivisible and discrete atoms different in kind from space, were adopted as scientific postulates, and were expressed in mathematical form. The following century was distinguished by a separation of philosophy and physics, as that period is best known by the extraordinary rise of the experimental method and the classification of phenomena under mathematical laws. With the mass of experimental data now at our disposal, an imperative need is again felt for theoretical laws which shall classify them, and accompanying this correct scientific need there is a disposition to re-introduce metaphysical systems, similar in aim to that of Descartes. The reason for this 
change in method in the nineteenth century is understood if we consider the state of scientific knowledge before that time.

Few of the properties of heat, light, sound, and electricity were then known, but, on the other hand, the laws of mechanics were well established, and a solid foundation of experimental fact permitted a broad and comprehensive application of pure mathematics to that branch of physics. It is altogether natural that mechanics should have developed first, for it is the only part of the science which rests directly on the data of experience. It considers only material bodies and their sensible and common properties-such as the occupation of space and the resistance to motion. To measure properties of matter other than spatial and dynamical requires more elaborate apparatus, and it is more difficult to separate extraneous accidents from such attributes as color, temperature, and tone. We cannot, even in the present state of mathematical knowledge, discuss the complex processes of nature as they are presented to us; for example, a mathematical law which shall define all the changes of color, of electrical intensity, etc., which occur when a body is heated, is still beyond our powers. But it was possible, with the knowledge then at hand, to abstract from matter all its properties except that of a simple and uniform space and force attribute, and to derive a theory of mechanical action distinct and complete. And so the 
philosophical scientists of the French revolutionary period, with whom this mechanistic movement culminated, had only mechanical problems to work on, in which their knowledge was practically as accurate as it is to-day.

However we may regard the effect of the metaphysical system of Descartes on science, there can be only one opinion as to the value of his introduction of geometry into physics. By it, he changed medieval natural philosophy into modern physics. When he discovered the method of locating the position of any point by giving its distance from three rectangular straight lines, he made it possible to represent the path of any moving body by a geometrical line referred to the same axes, which could then be fully defined by an algebraic equation; he thus made it possible to classify all motions under a few general types, and so founded the science of kinematics. For example, it became no longer necessary to study projectiles individually, for their behavior could be foretold from the general properties of parabolæ. Shortly after this, Newton and Leibnitz invented the calculus, which enables us to study moving bodies during their transit. The solution of problems of continuous motion in curved lines could never be satisfactorily obtained by Euclidean geometry, which could not overcome the break between rest and motion, or between polygons and curves. But the application of the infinitesimal calculus to Cartesian 
geometry gives us a solution of the problem, which, while it may not be the true solution, approximates to the truth as nearly as we please. This is accomplished by dividing the path of a moving body into sections so small that the ratio of any of these infinitesimal distances to the time necessary to traverse it, or the velocity, is constant and finite. Newton had discovered and verified the law of universal gravitation, and had also collected the data of the action of forces on bodies, and embodied them in three laws which still serve as the postulates for the science of dynamics; and D'Alembert supplemented them by a general dynamic law of the motion of a system of bodies acted upon by forces which embraced all the hitherto isolated problems of this character, and reduced them to a special and simple case of statics.

On the theoretic side, Kant, Lagrange, and others had discussed the general axioms of mechanics, and had established the three fundamental units-length, mass, and time; and it is generally conceded that the solution of any problem of mechanics into the simplest combination of these three quantities is incapable of further reduction.

But one thing remained before an imposing structure could be raised which should withstand criticism, and that was a general law to include and solve problems relating to a system of bodies in equilibrium and at rest. And Lagrange accomplished this. 
Such was the state of science when Laplace, in his Système du Monde, and Lagrange in the Mécanique Analytique, attempted to construct a theory and history of the universe by means only of the general and accepted laws of the two mechanics: celestial, which concerns the heavenly bodies, and terrestrial, those on the earth. Their problem has been stated in many ways, but this may serve:-Given the positions, masses, and forces of a system of bodies at any time, to find the configuration of the system at any other time, previous or afterward.

By the aid of the principle of centers of inertia, each celestial body could be replaced by a mathematical point, at which the whole mass was concentrated, and endowed with a force of attraction according to Newton's law of universal gravitation. In a similar way each terrestrial body was divided into a great number of small elastic particles, or atoms. These were considered to be invariable and indivisible, and they were arbitrarily endowed with the same force of universal gravitation, acting through their centers of inertia. This force could be assigned to these imponderable masses, separated by insensible distances, with some probability of truth, although it could not be scientifically verified by experiment, because it was known to be a property of ponderable bodies separated by sensible distances. This conception of matter was generally accepted, as the original atomic theory of 
Democritus had been extended and adapted to mathematical analysis by Gassendi, Huygens, and Boscovich, Thus all bodies and systems of bodies became abstractly alike in character, a collection of mass points acted upon by an attractive force common to all; and if the state of the universe were given at any time, it became merely a problem in mechanics, whose laws are fully known, to find its history from the beginning to the end. As Laplace proudly and naively answered: "In this system there is no need of a god." Evidently this statement was a climax of materialism, and probably can never again be uttered with such assurance.

So solidly was this theoretical universe built, that it defied criticism for a century, and finally, as it seemed, established science on a mechanical basis. The other branches of physics, which advanced rapidly during the nineteenth century, fell promptly under the influence of this mechanistic idea. The names employed show this clearly. We have the wave theories of light and sound, the dynamic theory of heat, and the mechanical theories of electricity and magnetism. In all these theories, attributes of matter, such as color, temperature, musical pitch, electrical charge, etc., are expressed by the mechanical motions and forces of atoms, and are measured solely in terms of the mechanical units of length, mass, and time. The method absolutely eliminates our senses, not only as instruments capable of measuring the quantity of an 
action, but even denies them the power of deciding qualitatively between phenomena; for the light which affects the eye, the sound heard by the ear, and the heat perceived by temperature are essentially the same thing, mere variations of a universal force of gravitation. These different attributes of matter are scientifically identical if the forces involved are equal; for as force, however manifested, was held to be a mechanical attraction between atoms, all these quantities can be weighed in a chemical balance and have no essential difference. While there may have been great diversity amongst the physicists of the last century as to details, there was but this one explanation of nature: The universe was a complicated machine, whose visible parts were connected together by a system of intangible links called atoms, whose complex motions, while they might defy our analytical skill, were yet completely expressible by general mechanical laws.

To find the weak spot in this mechanistic theory, based on the hypothesis of the atom, is not only a difficult task, but is one which ran so counter to the accepted teachings of science and to the natural prejudices of the mind, that it is not strange if most men of science now reason as though the atom were a matter of experimental proof rather than of metaphysical speculation. Such a mechanistic theory of natural law as Lagrange and Laplace evolved, and as scientific thought of the last century extended, must necessarily 
depend on some similar atomistic hypothesis. Complex material bodies must be divided up into elementary masses so small that any conceivable variation in them, except mere inertia, must be forever beyond our measurement or even conception. Because, if the atom were divisible or variable, which its very name denies, then the actions of its component parts and their variations might be productive of such an attribute as temperature or color, and thus introduce into the atom properties other than those purely mechanical.

From experience we know of only one way a tangible body may make another move, and that is by a direct push. Either atoms must be granted a mysterious power of attraction through empty space, or else the part of the universe unoccupied by ponderable matter must be filled with a medium or ether, to act as the mechanical link between atom and atom. Now this ether is either continuous or discontinuous. If continuous, it would serve as a link; but how is matter to move through it or even to exist in it unless two bodies may occupy the same space at the same time, or unless ponderable matter is but an attribute of this ethereal matter, of some such nature as a whirlpool on the surface of water? Such a variation maintains its identity of form as it moves, but not of the matter composing it; this is to replace concrete matter by the abstract idea of form or motion, which, in the end, is always repugnant to our sense of reality, and arouses 


\section{SCIENCE AS A SYMBOL AND A LAW II}

the impression of an existence in a world of dreams. On the other hand, if the ether be discontinuous, it must be porous, and what becomes of our link between atoms? We are driven to the creation of a second more tenuous medium to fill the spaces between the grosser one, and so on to the reductio ad absurdum pointed out by Clifford.

This discussion may be readily summarized in two metaphysical hypotheses which are frequently accepted as the axioms of the atomic theory.

First.-Given the masses and the configuration of the centers of inertia of all the atoms, with the law of their mutual attraction, then all the attributes of matter are determined and the problem of the universe is solved.

Second.-As a visible link is required between moving parts of a machine, so invisible links, called ethers, multiplied indefinitely, must exist between atoms.

While most scientists were endeavoring to extend and to perfect this mechanical theory, there were a few inclined to question the validity of the axioms on which it rested. Among the latter, Rankine deserves the first place. In a memoir read before the Philosophical Society of Glasgow in 1855 , he discusses scientific methods in general, points out the defects and advantages in the prevailing theories, and outlines a new method which he calls the science of energetics. His criticism is of the highest importance; with sub- 
tile irony he exposes the absurdity of a materialistic theory derived from mechanics which itself inevitably rests on a purely metaphysical basis.

According to Rankine, a true physical theory is the most simple system of principles from which the formal laws of phenomena, experimentally discovered, may be deduced. Such a theory resembles a science like geometry in that it originates with definitions and axioms for first principles, and derives their consequences logically, by propositions. But, in general, a physical theory differs because these fundamental definitions and axioms discovered first are numerous and complex, since they are deduced from the mass of facts presented to us immediately by nature; whereas the first principles and axioms of geometry are few in number and simple in character,-such as a mathematical line has length only,-and are the results deduced from bodies not necessarily real. In other words, the method pursued in the physical theory is inductive, and is consequently more tentative and laborious than the deductive method of geometry, as the acceptance or rejection of the principles derived must depend upon their agreement with facts discovered gradually by observations, and not upon general properties agreed upon once for all. The propositions of geometry are final, if the axioms and definitions are granted; a theory of physics is more or less conjectural, as its first principles are always subject 
to revision because of the discovery of new phenomena.

Two methods of framing a physical theory may be distinguished. They may be termed the abstractive and the hypothetical methods.

According to the abstractive method, a class of objects or phenomena is described and a name or symbol assigned to that assemblage of properties common to all the objects or phenomena composing the class, as perceived by the senses, and without introducing anything hypothetical.

There is only one example of a complete physical theory formed exclusively from the data of experience by the abstractive method,-the principles of the science of mechanics. The objects discussed in mechanics are material and real bodies, all of which possess the sensible properties of occupying space and resisting change of motion. The phenomena dealt with are confined to those attributes of matter distinguished by the words force and motion, which we have found to be common to all bodies of which we have any knowledge. And the laws deduced follow from axioms and definitions which express this universal experience.

According to the hypothetical method, the existence of a class of fictitious objects or phenomena, which cannot be perceived by the senses, is assumed. And properties are assigned to them, similar to those known to be true of a class of real objects or phenomena, which 
can be perceived by the senses. If the consequences of such a hypothesis are afterwards found to be in agreement with the results of observation and experiment, then the laws, known to be true for the class of real objects or phenomena, may be applied to the hypothetical class. The objects or phenomena considered by this method are thus merely matters of conjecture, and their nature may be modified at any time so as to make the propositions derived from them conform to an expression of experimental fact. Such, for example, has been the method followed in the wave theory of light. To explain the observed action of light, the existence of hypothetical bodies, called atoms, and the luminiferous ether, is assumed, and properties are assigned to them similar to those of sensible matter. As new phenomena are discovered the attributes of the atom and the ether are modified to fit the requirements. This theory can be considered only as a convenient means of expressing natural laws, and is always subject to change, as it does not depend on the objective realities fundamental to an abstractive method.

Just because the theory of the mechanical motions and motive forces of sensible bodies is the only complete physical theory, and because it does not require the use of a hypothetical method in its development, we have been led to give the hypotheses, advanced as theories in the other branches of physics, a mechanical form. The classes of phenomena considered in all 
these theories are defined conjecturally as being due to some kind of mechanical motion and motive force, as when heat is defined as consisting in molecular motions, or the rigidity of solids in molecular attractions and repulsions.

The motions and forces involved in these theories can no longer be ascribed to sensible matter, but either hypothetical bodies, such as the luminiferous ether, or hypothetical parts of real bodies, such as molecules, atoms, ethereal vortices, or other imaginary elements of matter must be created. And to them are assigned properties and laws resembling as closely as may be those of sensible bodies. In explaining new facts, as they are discovered, the attributes of the hypothetical matter are modified, or such new ones assumed as may best fit the case. Such mechanical hypotheses, not being based on experimental evidence, are held to fulfill their purpose when these conjectural attributes explain in the simplest and most plausible way the largest body of known phenomena and when they anticipate phenomena afterwards observed. The importance and weight of these hypotheses increase with the number of phenomena whose laws they express.

Certain hypothetical theories, such as the wave theory of light, have been undoubtedly useful, since they have reduced complicated actions to a few simple laws. And also they tend to combine all branches of physics into one system in which the axioms of 
mechanics are the first principles of the whole science. But they must be employed with great caution and judgment. Their free use tends to confuse the essential differences between hypothesis and fact, between metaphysics and physics, and this confusion does now exist in the minds of the public generally and even in those of many men of science. A desire is, consequently, often shown to explain away, or set aside, facts inconsistent with a preconceived hypothesis.

Such is briefly Rankine's criticism of the prevailing mechanical and materialistic theories of physics. His conclusions are worthy of thoughtful consideration. It has always been the boast of science that by its methods we may avoid the pitfalls in which metaphysical reasoning inevitably ends. Now, if our most elaborate and complete scientific theory is really metaphysical, we must renounce all our proud claims and consider atomic and mechanical theories solely on the grounds of their utility and simplicity.

A metaphysical hypothesis, valuable solely for its utility, is always dangerous, for by constant use we tend inevitably to give an objective reality to things which in the beginning we knew to exist only in our own minds. And this tendency is especially deplorable in science, which does little for education if it does not recognize clearly the limits of our knowledge and distinguish accurately between reality and speculation.

Now the belief in the objective reality of molecules, 
atoms, ethers, and ethereal vortices has grown so steadily that little objection has been made to the recent creation of a whole new class of such hypothetical objects, called indifferently ions, corpuscles, electrons, or particles, which are assumed to be the constituent elements of the hypothetical atom. Of the three classes of objects it is, at the present time, the existence of the sensible bodies which is in danger of repudiation. This is the case not only in the minds of the thoughtless but in those of the leading men of science. For example, Sir J. J. Thomson, in the preface to his Conduction of Electricity Through Gases, says: "The possession of a charge by the ions increases so much the ease with which they can be traced and their properties studied that, as the reader will see, we know far more about the ion than we do about the uncharged molecule." Such a statement is on a parallel with the remark made to me by another distinguished physicist, that we know far more about the ether and the atom than we do about sensible matter. This is true, and in the same way as a Frankenstein might say of a mechanical man which he had conceived and constructed,-I know more about him than I do about a real man.

Such confusion of thought is directly traceable to the fact that many men of science have forgotten the distinction between the creations of nature and the creations of their imaginations. We can never say 
more of molecules, ions, and the ether, than that they may exist; but ponderable matter, as perceived by the senses, has an objective existence, or else there is no place for science. Since Kant's time the existence or the non-existence of those insensible links in the universal machine is known to be equally demonstrable; we have no criterion of proof. It is curious that we still refuse to acknowledge this. If we look askant at Kant, the metaphysician, we have only to turn to Lagrange, whose scientific claims cannot be ignored, and find he has proved by rigid mathematical analysis that any phenomenon, which obeys the law of conservation of energy, is capable of an explanation by a mechanical theory; but, and here is the important point, as there is always one adequate theory, so there are also an indefinite number of other mechanical theories which will, so far as our minds are concerned, satisfy all the requirements of the case. We have no criterion in mechanics by which we may determine what is the actual process of nature. There is no experimentum crucis, and we choose the explanation which for the moment seems to be the simplest.

Our inability to decide unequivocally for one mechanical hypothesis instead of another is shown also by the actual history of physical science. Since the time of Huygens and Newton we have attempted to settle the question whether light is due to a wave motion in an ether or to small particles emitted from 
SCIENCE AS A SYMBOL AND A LAW 19

luminous bodies. Many times it has been announced that the question has been decided experimentally; for instance, when Foucault found that the velocity of light decreased when passing through transparent bodies, instead of increasing as Newton's hypothesis had predicted. But, on the other hand, the aberration of light discovered by Bradley is a stumbling-block to Huygens' theory and is a simple consequence of Newton's. Neither of these experiments caused the abandonment of a theory. A Newton could have altered the properties of the corpuscle and a Huygens is sure to arise who can alter the character of ethereal waves so as to explain aberration; in fact, it has been done with considerable success by Sir Joseph Larmor. We may then take it as established, both on theoretical and on historical grounds, that no experiment has been, or will be, devised to decide finally between the claims of the two hypotheses, yet the corpuscular theory was abandoned. The reason was not that either was impossible, but that the corpuscle, with the accretions added to it as new facts were discovered, became too unmanageable. Huygens' mechanical wave theory having outgrown its usefulness has suffered the same fate. He ascribed light to a series of mechanical waves propagated through an elastic ether, but the attributes necessary to the medium became so contradictory that a new theory, advanced by Maxwell, was accepted as a great relief. In this theory, the ethereal 
waves are not mechanical but electro-magnetic, similar to those we now use in wireless telegraphy. But the difficulties are still pursuing us. We may assume that such waves can pass through space, but we cannot construct a mechanical model of an atom or ether which will produce or maintain these vibrations, nor have we any evidence that electro-magnetic disturbances can affect the optic nerve and produce the sensation of light. The prediction, which I made when this essay was first published, that we shall return to a modified form of the corpuscular theory with the electrified particle, the constituent of the atom, as an agent, has been verified. The principle of relativity, recently announced by Professor Einstein, absolutely requires it.

If a general atomistic theory, which seems to be the only practicable hypothesis, involves these inherent difficulties, and if it presents a real peril to correct scientific thinking, the question arises, whether some general mechanical explanation of all physical phenomena is possible which is not so limited.

Rankine, in the same essay, proposes a method which he calls the science of energetics. As we have been able to frame with some success a theory of physics by using a hypothetical method, we should have even more success in combining all the branches of the science into one general theory if the abstractive method were extended and applied for the purpose. Instead of supposing the various physical phenomena to be con- 
stituted, in an occult way, of modifications of mechanical motion and force, let us attempt to frame laws which shall embrace the properties common to any one class. He finds energy, or the capacity to effect changes, to be the common characteristic of the various states of matter to which the several branches of physics relate. If then we frame general laws regarding energy, we shall be able to apply them, with appropriate changes, to every branch of physics.

Rankine evidently denies the advisability of trying to find the cause of the attraction of bodies for one another, or the mechanism of the propagation of light and heat through empty space. In all cases we have a certain quantity of energy, acting in a specific manner. Our aim should be to find by experiment the properties of any such manifestation, and to combine all common properties by general mathematical laws. Such was the method of Newton when he established the law of universal gravitation and refrained from publishing how the forces of attraction could act through space, and no discovery has aided science more. But after he had determined experimentally many of the laws of light, he advanced the hypothesis that these phenomena were caused by motions of intangible corpuscles and he ventured so far as to describe the shape and properties of these hypothetical bodies. It is claimed, on good grounds apparently, that his corpuscular theory retarded the growth of the subject for more than a century, by 
preventing the adoption of the temporarily more convenient wave theory.

Whether or not it is advisable to substitute energy for inertia, or mass, as the general attribute of matter which will best serve for a fundamental unit, may be open to discussion. But it seems certain to me, at least, that the formulation of laws deduced mathematically from experimental data alone, and not conjecture as to the causes of phenomena, is the true province of science and the only method certain not to lead us into vain metaphysical speculation.

Unfortunately, the restraint and clarity of thought shown by Rankine are rare, and few are willing to impose limitations on speculation or to forego the attempt to create a subjective and metaphysical scheme according to which nature shall work. In the hands of his successors, notably Mach, Duhem, and Ostwald, these barriers were cleared. They have endeavored to give an objective reality to the mathematical equation of energy. To make an entity of a symbol, to speak of centers of force as if an intelligible image were conveyed to the mind, to make matter and inertia an attribute of energy, is even more metaphysical than the concepts of atoms and ethers, which could, at least, be likened to sensible objects. With Professor Ostwald, the most militant defender of the science of energetics, matter disappears altogether; empty space is known to us only by the quantity of energy necessary 
to penetrate it, and occupied space is merely a group of various energies. In his enthusiasm he does not hesitate at difficulties. "When a stick strikes you," he exclaims, "which do you feel, the stick or the energy?" One might as well ask the old question, Which comes first, the owl or the egg?-a matter of infinite dispute and no decision. Although Professor Ostwald's work bristles with mathematical equations and scientific terms, he asks us to return to the metaphysical methods of the medieval schoolmen-to thrash over again the endless verbal disputes of nominalists and realists.

While mechanics is the only branch of physics which has become a complete science through the use of the inductive, or abstractive, method, just as soon as we invent atoms and ethers, or consider an abstract quantity, like energy, to be an entity, in order to explain the modus operandi of matter and motion, and to serve as connecting links in explaining non-mechanical phenomena, such as electricity and light, mechanics drifts at once into a highly metaphysical and subjective study where each man's opinion is guided only by an inward sentiment of knowledge. To what lengths this can go, I shall show in a later chapter when I present the confusion of thought of certain eminent men of science who change impersonal mechanical energy into a kind of vital and beneficent principle ruling over the thoughts and actions of human society, 


\section{THE LIMITATIONS OF SCIENCE}

or of Sir Oliver Lodge, who bestows on the ether the ability to carry ghostly messages as well as light.

As a critical attempt, the school of energetics has done good work by calling attention to the inadequacies of atomic theories, yet as a positive method it has had comparatively little effect until very recently, when it has been pushed by a school of German physicists into what can only be called an abyss of confusion where, as in a sort of looking-glass world, all things tangible become intangible and the abstract replaces the concrete. But the majority of men of science still rely absolutely on atomic hypotheses. Indeed, a fresh stimulus has been given them by the efforts to explain the experimental facts, recently discovered, concerning Roentgen rays, the passage of electricity through gases, and the properties of radium; facts which will probably do more, in the end, to discountenance mechanical models of phenomena, by making them practically unmanageable, than the theoretical criticisms of the followers of the school of energetics.

So long as the hypothesis of an invariable and indivisible atom gave a reasonably simple and satisfactory method of attacking the problems of physics, even those men of science who were ready to acknowledge the tentative character of the hypothesis and the contradictory nature of its postulates were unwilling to try other methods. But the phenomena mentioned above do not fit into the general scheme, because the 


\section{SCIENCE AS A SYMBOL AND A LAW 25}

mechanical atom does not explain such electrical phenomena, unless we arbitrarily associate with it other supposititious electrical fluids or else give it complex and variable electrical attributes; both of which defeat the purpose of explaining all phenomena by means of a single, invariable entity. Their explanation thus requires us either to abandon the atomic theory or to modify it radically; the latter has been done, and the atom is now supposed to be a complex body composed of an aggregation of invariable and indivisible particles, called electrons.

As might be supposed, some specious advantages have been obtained. The chemists have long sought in vain for a chemical element whose atom might be considered the primordial substance, and from which the atoms of the other elements were derived. This new idea of the atom offers a solution, for the chemists may now construct the atoms of all the elements out of different combinations of corpuscles. Also the early investigators in electricity, as Franklin and Du Fay, were led to postulate the existence of subtile electric fluids to explain the fact that electrified matter sometimes showed a force of attraction and sometimes of repulsion. Later, in the theories of Faraday and Maxwell, the hypothesis of fluids was abandoned and the ends of the atom of matter were endowed respectively with the properties of electrical attraction and repulsion. Now it is possible to discard this 
variability in the simple atom by supposing some of the corpuscular elements of a complex atom to exhibit the one kind of electric force, and others the opposite kind.

In spite of these advantages and others which might be cited, the prime fact remains that it is now necessary to abandon the historic and hitherto invincible atomic theory for another which is still more conjectural. For the former element of matter, simple in nature, we have substituted another, complex in character, and have thereby given up the chief and to many the only value of an atomic theory.

The corpuscular theories advanced, almost simultaneously by Lorentz and Larmor, show this clearly. Sir Joseph Larmor, in his treatise on Fther and Matter, presents a view of the constitution of matter which is sufficient over an extensive range of physical theory, and which he trusts will not be made more complex until it proves inadequate in some definite feature. According to his hypothesis, the atom of matter is composed of a system, probably large in number, of positively and negatively electrified protions (called frequently by others corpuscles, electrons, or ions) in state of steady orbital motion around each other. The passage of electricity through a conductor or from one body to another is effected by a transference of electrically charged protions from one atom to another. The differences in the chemical elements, such as iron 
or hydrogen, can be accounted for by ascribing them to various aggregations of the protions. As for the protions themselves, they are in whole or part nuclei of intrinsic strain in the ether, places where the continuity of this medium has been broken and cemented together again.

Such a theory is evidently, and in the highest degree, artificial and metaphysical, and Sir Joseph Larmor would be the last to assert that he has given a true picture of the constitution of matter. Its value must rest on the belief that it is the simplest theory available for explaining experimental facts. But the difficulties inherent to the theory are insuperable. It is almost inconceivable that our simplest idea of the ultimate constituent of the chemical element should be an atom, so bewilderingly complex in character. Each atom of an apparently quiescent body is itself an aggregation of particles, vastly more intricate than the stellar systems, and whirling around each other with a motion approximating a hundred thousand miles per second. And although the atom itself still possesses the attributes of matter, its constituents become merely nuclei of strain in the ether. What must be the structure of an ether which can maintain such a complex of strains as all the countless atoms in the universe would require? If we can never be sure matter is actually so constituted, it is unfortunate to create a world so counter to our instinctive belief that in a 
correct definition a complex idea must be explained into simpler parts.

The theory of Professor Lorentz is essentially the same, although he does not attempt any speculations as to the structure of the ether or atom. But he, too, postulates the existence of small, electrically charged particles in all bodies and deduces all electrical laws from the positions and motions of these electrons.

It is not necessary to state that both these writers develop their theories with great skill and from a profound knowledge of the science. They have, perhaps, achieved a closer unity in the branches of physics, although I feel confident that they have really only substituted an electrical atom which will not explain matter for a material atom which would not explain electricity. And to attain this ambiguous advantage they have introduced postulates which lie still further outside the domain of science and have, by fixing our attention on a sub-atom, given an appearance of greater reality to the relatively gross atom.

The influence of such abstruse and metaphysical theories on scientific thought is already apparent in a certain eagerness to advance startling hypotheses and novel ideas. Many men of science of to-day have temporarily put aside the sobriety and restraint which should characterize scientific reasoning. The most tremendous results are based on insufficient evidence, and the simple statement that the cause of a phenom- 
enon is to be found in electronic action is considered satisfactory. Physicists in Germany are gravely discussing whether electrons are spheres or discs in shape. The transmutation of the elements, a problem which has baffled research for centuries, is announced as an assured fact, because radium and a few other substances spontaneously give off energy. Electricity is declared to be a substance and matter is said to be not a substance, but an attribute of electricity, because an electrified body has an apparent increase in its resistance to motion; although we know nothing about the nature of either matter or electricity. Others say that the universe is a sort of modified ether; although we never can have cognizance of an unmodified ether. Surely matters of such infinite difficulty and of such supreme importance should not be decided before the most rigid elimination of more natural causes. The fact is, all such statements as these are merely words which convey no ideas; the problems involved are entirely beyond our powers of solution either by physical or by metaphysical methods. Such confusion of thought and dissolution of the boundaries between fact and fancy are deplorable, and if they create trouble in the minds of scientific men, they have absolutely bewildered the general public. Books of a popular nature are constantly appearing which change these results of speculation into established fact, and their readers naturally credit the most astounding state- 
ments. The day may come when a new war will arise between science and religion on the issue that the deductions of science are too metaphysical to be of value.

It may be necessary, when the laws and phenomena of a science are vaguely known, to employ a hypothetical method. And a hypothesis may then be of great use in creating a certain unity amongst diverse elements. But the question may well be asked, whether physical science has not outgrown a method proper for the alchemist and the astrologer.

The attempt to unite the phenomena of all branches of physics in a few general laws and to explain their cause by the aid of atoms has engaged the attention of the greatest men of science for more than a century. They have spent upon these problems infinite thought and pains, and in the end we have a body of laws firmly established on experimental evidence, but the causes of these laws are as hopelessly obscure as ever. The atom has failed to satisfy the requirements, and now the electron is added to explain new facts, an hypothesis on an hypothesis. As our knowledge increases, who can doubt but that these, in their turn, will give place to others still more complex, if the same method is pursued, until the succession of atoms and sub-atoms will make the whole atomistic idea an absurdity?

Just as we have, after centuries of incessant controversy, been forced to accept the fact that we cannot by reasoning from our consciousness obtain an ob- 
SCIENCE AS A SYMBOL AND A LAW 31 jective knowledge of natural causes, so we must come to realize that reasoning from experimental evidence is subject to exactly the same limitations. Science, in other words, like philosophy, has no ontological value. Should not the men of science clearly recognize this fact, and confine their effort to the legitimate function of science-the discovery of natural phenomena and their classification into general laws derived by logical mathematical processes? 


\section{CHAPTER II}

\section{THE METAPHYSICAL TENDENCIES OF MODERN PHYSICS}

Però, signor Simplicio, venite pure con le ragioni e con le dimostrazioni vostri o di Aristotile, e non con testi e nude autorità, perche $\mathrm{i}$ discorsi nostri hanno a essere intorno al mondo sensibile, e non sopra un mondo di carta.-Galileo.

IT is a more or less simple thing to discover and follow the main current of thought in a science like physics, which must develop logically or not at all, after the confusion of strife has passed away and only the permanent additions to our knowledge remain. This has been attempted in the former chapter, so far at least as the atomic theory is concerned. It was then shown that if we build up a homogeneous hypothesis of the natural phenomena-heat, light, sound, and electricity-from an analogy to mechanical laws, we inevitably postulate the objective existence of matter, and create a series of fictitious ethers, and of atomic, or indivisible, elements of matter. The claim was also made that this method which attempts to explain the laws of nature not only fails to do so, but also prevents the adoption of a better scientific procedure.

In the first place, it is difficult to point to any scien- 
tific discoveries directly and inevitably produced by a specific atomic theory. The contrary of this opinion is very generally held, and many such discoveries in chemistry and physics are laid at its door. The chemist searches for and combines new compounds of the elements, and bases his theory on the assumption that each element is disintegrable only to a fixed atom. But this means nothing more than to say that the elements combine in definite proportions of mass, and consequently does not bear on the question whether matter is infinitely divisible. The chemist would have been driven to the same laws of chemical combination if he had believed matter to be infinitely divisible. This statement is true, because chemical analysis and synthesis progress imperturbed and as rapidly now when the chemical atom is supposed to be decomposable. And the same was true of chemical progress before Dalton proposed his atomic theory, which besides was so simple in form as to be little more than the generalization, that from experience we may say chemical substances unite in simple multiples of a unit chemical mass, called the atom, instead of simple proportions of mass. That is, Dalton's atomic theory was more closely related to the abstractive than to the hypothetical method and really has little in common with the physical atomic hypothesis which gives definite and complex functions to the atom. As examples in another field, we find the phenomena and laws of the 
double refraction and polarization of light were discovered by Huygens before he, and later Fresnel, attempted to explain them as a modification of mechanical waves in an elastic solid ether; and Newton announced the laws of the interference of light which occurs when there are reflections between thin films before he pictured this phenomenon by his hypothesis of corpuscles. To-day all these laws remain while the specific hypotheses have been discarded. How can we say the hypothesis of atoms and ether led to discovery in these cases? The fact is just the contrary: hypothesis, at least that part of it which consists in developing a mechanical model of the action, follows experimental discovery; it is the effort to explain or visualize the unknowable processes involved in known experimental facts and mathematical laws.

In the second place, these metaphysical hypotheses progress from the simple to the complex. Each new fact discovered adds its quota to the irreconcilable and conflicting properties of the ether and the atom, and these invisible links of the universal machine grow more and more bewildering and complicated, until the whole construction falls to pieces. Nor is this all; the man of science forgets that he is building toy houses, and ends by believing in their reality. Even if hypothesis does not carry him so far, it certainly has this effect on others who accept the dogmas of science without discrimination. It is no small danger thus to 
confuse reality and imagination; a science, which becomes so hypothetical or so specialized as to be unintelligible to the educated man, is apt to become as sterile as a religion which is in the sole possession of a hierarchy.

This excessive use of hypothesis has developed a sort of scientific cult which somewhat resembles a religious dogma, in that adverse criticism of either arouses a feeling of personal irritation. The rancor of religious polemic is well known and is said to be due to the fact that the believer of a religion relies on revealed truth, to doubt which is sinful. The same occurs with the supporters of a scientific hypothesis, who declare their system to be founded on objective, experimental fact, and to be developed by logical methods, so that in doubting the hypothesis we are sinning against truth and reason,-the gospels of science. On the other hand, discussions in experimental science are noted for their calmness, for then we are criticising, not personal opinions but objective facts, and we care comparatively little which way the matter ends. The theorist, on the contrary, forgets that, while founded on experience, his hypothesis is developed in one way or another according to his own personal opinion; for example, the same facts of light made Newton believe in corpuscles and Huygens in waves, and so the theorist injects into his discussions the bitterness of personal defeat or the exultation of personal victory. 
There is yet another purpose in science which requires simplicity instead of complexity. Physics, to be something more than an intellectual puzzle for the specialist, should enlarge our power over the external world and increase our use of natural resources. Consider how great an advance we might make in this direction if hypothesis and occult causes were reduced to a minimum. Instead of a mass of abstruse speculations on the nature of ether and matter, our treatises might present a clear and logical discussion of natural phenomena and their laws. The work of Lord Kelvin is typical. He has interspersed in his writings probably a score of models of the atom: now it is a vortex or whirlpool in a continuous fluid ether; now, a box containing gyrostats or wheels spinning on axes; again, it is a complicated structure of balls, strings, and springs. No two of these agree in principle, and at best represent crudely a limited number of the properties of matter and fail for others. Is there not, after all, something almost pathetic in this incessant striving of the greatest physicist of our times after the unknowable, building card houses which must be knocked down to provide material for new ones? Certainly more of his great and permanent constructive work would be the property of the world if we had neglected his hypotheses and developed more diligently his experiments and his laws.

On the other hand, it is a serious matter to try to 
sweep aside so large a part of scientific thought as hypothesis has been, unless it is really parasitical,-a hindrance rather than an aid to development. This opinion as to the uselessness of hypothesis seems to be gaining ground. Thus M. Duhem, in his Théorie Physique, states that physical theories must have one of two aims: either to explain laws which have been established from experience, or to classify such laws without giving any explanation. Of the two, the second only is a legitimate scientific process, as the first method makes physics dependent on metaphysics and so introduces occult and unverifiable causes. A proper theory should thus give us a classification of laws and should point to new experimental methods, thereby tending to intellectual economy in that we are permitted to forget a multitude of details and otherwise isolated facts in one common expression. He further claims that the construction of a mechanical model as an explanation of a law does not lead to such discoveries, since these are really derived from abstract principles, the model being invented afterwards merely to make the law concrete. In this opinion he is supported by Hertz, who, after discovering experimentally the electric waves predicted by Maxwell, found the best statement in Maxwell's equations, and not in his model of ethereal lines of force.

A ruthless and complete elimination of hypothesis is undoubtedly impossible, and is not even desirable. We 
tend invariably to express our abstract conclusions in a more or less concrete form. We shall always speculate about the manner of the propagation of light to the earth from the sun, and it is convenient to express this transfer of energy either as a periodic disturbance taking place in something filling space or as something projected through space. Such indefinite speculations are simple enough and allow us to state all that we need in order to assume continuity of action in free space. The obscurity and confusion in the science of optics arise from the attempts to express in detail the nature of the waves or of the particles of light. A method where speculation is kept to its lowest terms is quite different from the prevalent custom 'of spending the greater part of our effort on the fictitious properties of the ether rather than on the phenomena and laws of matter.

The problem of tracing the tendencies of thought concealed in the conflicting data and opinions of the present state of physics is difficult, and this difficulty is increased by the unusual amount of new material brought to light since the discovery of the X-rays by Professor Roentgen in 1895 . The phenomena connected with the discharge of electricity through gases and with radio-activity are obscure, and speculation about them correspondingly bold. There is a desire, quite common, to ignore the importance of the more regular advance in other branches of physics and to 
assume that the new methods of attack which have arisen are essentially different from the thoughts and speculations of former physicists and not subject to their failure. This idea is brought out by Mr. Campbell in his recent book on Modern Electrical Theory, when he contrasts the work of Faraday, about I830, and his predecessors with that of living physicists: "Men of his own and of the preceding era had founded 'natural philosophy'; they had made discoveries and had elaborated theories which still form part of the frame-work of the physical sciences. But their work has little interest for us to-day. Their aims, their conceptions, their whole attitude toward the problems which they investigated differ so widely from our own, that, while their results may be the basis of modern research, their methods afford little inspiration for it." This is far from being the case; if we can assign definite periods to so continuous a development as the history of science has shown, we should certainly date modern physics from the seventeenth century when Galileo introduced experimental methods and Descartes applied analytical geometry to physics: human thought does not progress at this late day by cutting loose from the past; especially when that past is said to date from the middle of the nineteenth century. Where the "new views" are not merely statistical observations or fugitive models of some particular phenomenon-that is, where there is a philosophical 
background to our recent work-we have abandoned the ideas of the atomistic school of the nineteenth century only to fall back on the doctrines formulated by Descartes in the seventeenth, as Sir Joseph Larmor has pointed out.

To show this connection, it is necessary only to outline and contrast briefly these two methods of scientific procedure now, as I have already shown the tendencies of the atomistic school and shall give, in the next chapter, the ideas of Descartes. The followers of the atomistic school believe natural phenomena to result from the impact of atoms, possessing mass, figure or extent, indestructibility, and the inherent property of motion. Thus this idea, adopted by Huygens, agrees in the main with that of Newton, except as it rejects his hypothesis of the occult power of attraction of atom for atom through space. With Descartes matter, as a distinct and separate entity, disappears altogether, and nothing is left but space and its variations. What we call pure space or a vacuum is really a continuous fluid plenum or ether, and material bodies are merely places of permanent variation in this plenum. From observing the persistence of whirlpools in water and in the air, Descartes ingeniously concluded that all space was filled with whirlpools or vortices of this plenum, each having an axis which passes through one of the stars. Unfortunately for the theory, these vortices and material variations of space soon became so compli- 
cated as to destroy its value. In the last century the idea was revived by Lord Kelvin in his celebrated vortical theory of matter. He used only the main ideas of Descartes, and, from a better mathematical knowledge of the properties of vortices, was able to simplify their character and to account for many of the attributes of matter. We may then say that the dissimilarity in the two theories consists in the doctrine that empty space is a vacuum or an imaginary entity called the ether, as opposed to the postulate of Descartes that space is true material substance; while both theories suppose that the smallest particles of sensible matter are indivisible, the atomistic school considers them to be atoms, inherently indivisible and essentially different from space, but Descartes declared them to be variations of a substance, itself infinitely divisible, although they might not be further divisible by our present experimental ability. With this understanding of Descartes's hypothesis, we are in a position to show how closely modern views of matter and electricity are concurring in this idea.

From the large number of physicists now writing on the theory of physics, three names stand out prominently as originators of the modern conceptions of electricity and matter. Professor H. A. Lorentz, Sir Joseph Larmor, and Sir Joseph Thomson are certainly the men who will be most prominently associated with this movement; others have aided, but mainly in the ex- 
tension or modification of their ideas. And of the three, the most attention in an essay of this character, which attempts a general discussion of the philosophical basis of scientific theory, should be devoted to Professor Larmor's ideas. In his treatise, Ether and Matter, published in I900, we have the rather rare example of a scientific theory with a philosophical background clearly expressed and discussed.

The main thesis of his essay is that a purely mechanical theory of discrete atoms moving in empty space has failed to account for the phenomena of nature, and especially for the recent discoveries made in electricity. We may, however, by altering this conception of atoms, by separating electricity and matter into two entities, or even by considering matter as an attribute of electricity, again reconcile fact and hypothesis. Since Faraday's time the drift of opinion has been in the direction of this separation, so that it is merely necessary to crystallize it into a definite scientific postulate. No idea of finality is expressed in Professor Larmor's theory, since he thinks it should endure only so long as it agrees with facts in our possession; on the other hand, he considers it not to be effective criticism to make a charge of incompleteness without indicating a better way, as an hypothesis may be valuable not only when imperfect, but when quite wrong, providing it serves as a useful instrument for the progress of natural philosophy. As an 
instance of this, he states that many of the most important discoveries in light were made when the erroneous corpuscular theory was still in vogue. But does it necessarily follow that the theory prompted the discoveries or led to their investigation because they happened to be contemporaneous? It is difficult to believe Bradley would have failed to obtain the relation between the aberration of light and its finite velocity if some other theory had been popular. Certainly the specific attributes assigned to light corpuscles would have little influence in promoting such discoveries, since it was the habit to modify these without much compunction if they did not square with observation. And we are supported in this opinion by Professor Larmor himself when he says: "At the same time all that is known (or perhaps need to be known) of the ether itself may be formulated as a scheme of differential equations, . . . which it would be gratuitous to further explain by any complications of structure"; and again: "The ultimate inadequacy of a method of treating material media, based on merely empirical or speculative additions to the ascertained equations of free ether, had indeed been clearly recognized by von Helmholtz."

The questions, then, to be borne in mind while discussing these modern theories, are whether they involve speculative additions to our equations and explanations by complicated constructions, and whether they are 
essential to the progress of science. I shall try to show that they are characterized by the same occult and unverifiable assumptions as the older theories and are really extra-scientific.

Professor Larmor, in the beginning of his essay, recognizes that an hypothesis which supposes matter to be constituted of an immense number of discrete particles moving in empty space and incapable of further subdivision has a philosophical objection too difficult to be overcome. In the first place, as Lord Kelvin pointed out, the chemical atom cannot be the immeasurably small body sometimes claimed by metaphysicians. Both physical and chemical experience require the atom to be a real portion of matter occupying a finite space, and forming a not inappreciably small constituent of any palpable body. The chemical molecule may be decomposed into the atoms of hydrogen and oxygen, and now these atoms are in turn divided into subatoms. Even these also are by no means immeasurably small; we are already calculating their size and their mass. And we can think of no reason why matter should have been created of this size rather than any other. These minute grains still have much individuality of their own in the way of attributes; if electrified, their mass is supposed to change in quantity when they move, as does also their shape; they must explain electrical attraction and repulsion, gravitational attraction, cohesion, and a probable molecular repulsion 
known as elasticity; they must have a propensity which makes them build geometrical crystals: in fact they must be arbitrarily and occultly endowed with all the attributes of ponderable matter which they were created to explain. This sub-atom has many more duties to perform, but sufficient have been given to show that either it is excessively complex in essence or is endowed with complex forces; so far, this is our arbitrary resting point in the matter of subdivision, but it is merely a temporary makeshift. But there is a still more cogent reason for this philosophical objection than the empirical ones given. The equally fundamental concepts of space and time are invariably considered as continuous or infinitely divisible functions, and this theoretical difference assigned to matter, introduces inevitable trouble in mathematical analysis. The science of mechanics, in its theoretical aspect, may be defined as the attempt to apply the laws of geometry to real bodies, and while there is always an insurmountable break in thought between the laws of the imaginary bodies discussed in geometry and the concrete ones of mechanics, we can, by the assumption of the continuity and indefinite divisibility of matter, approximate as closely as we wish to the rigorous laws of geometry. The geometrical point and line are respectively abstract bodies of no, and of one, dimension; in experimental mechanics, they are both real bodies of three dimensions, - the point is the atom and the line 
a succession of them. Theoretical mechanics is thus the link between geometry and physics, for while it deals with concrete bodies of real size, we eliminate by the abstractive method all the variations which result from the finite size of bodies and then discuss them as if they were theoretically simple bodies of no size. That is, we divide a body into infinitesimal portions and consider its effect as being due to the arithmetical sum of the effects of these parts. An example will make this clearer; the attraction between two spheres, which is one of the fundamental problems of physics, can be solved rigorously only by the application of the principle of centers of inertia, that the attractive force of a sphere is the same as if its entire mass were concentrated at its mathematical center. As soon as we apply this law of attraction to real spheres, even of sub-atomic size, we introduce an appreciable error, since the attractive force then produces strains and a deformation of shape which reacts to change the force; this error becomes negligible only when we consider matter to be continuous and the sphere to be made up of an indefinitely large number of indefinitely small particles. We are thus brought to this dilemma; experimentally, matter is discontinuous and theoretically, it is continuous,- -hypothesis might be called the hopeless effort to reconcile these irreconcilables.

Professor Larmor tries to avoid this pitfall, which has caught the originators of atomic theories, and at- 
tempts to reconcile the antagonistic ideas of continuity and atomicity by placing himself squarely on the side of Descartes. He does not regard space as mere empty geometrical continuity. According to his notion, the universe is a plenum or ether - that is, a continuous, frictionless fluid, everywhere uniform and quiescent. This plenum constitutes what he calls true matter. It is entirely unrecognizable by our senses and cannot be brought to them by any experience. By such a metaphysical hypothesis we may account for the aberration of light and many other actions occurring in free space. To provide for ordinary or sensible matter, making it at the same time discontinuous in character, he supposes there exist in the plenum innumerable places of variation, which are uncreatable, indestructible, and humanly indivisible, and by their combination present to our senses all the phenomena making up for us the material universe. These discontinuities are free to move without disturbing the quiescence of the continuous medium, much as wind blows through a forest. By this supposition we account for the experimental fact that motion of matter does not affect the velocity of light in a vacuum. If, however, the discontinuities show unbalanced electrical force, then their motion causes real, although temporary, variations in the medium.

The next requirement in his cosmogony is to specify what these variations in the supposititious plenum may 
be. This is the vital step in any process of reasoning which attempts to link metaphysical assumption to physical experience. Once taken without challenge, a scientific theory may be developed logically. It is just at this point that the Lucretian atom and the Cartesian vortex fail. Here also Lord Kelvin failed. He proved that no finite force could either create his tiny vortex atom rotating in a frictionless medium, or, once started, could stop it. He showed it would act as if possessed of many of the essential properties of matter. But, in the process of elaboration, this atom, like all others, became unmanageable from complexity; it failed to account for the electric charges of matter, and finally received a death-blow when Maxwell said a vortex ring might be an analogy to the atom, but at best was merely a mode of motion and not matter as we know it.

How, then, is this new protoplasmic element of the universe to be defined so as to satisfy these criticisms, and at the same time avoid making the speculative mechanical structures in an ether, which Professor Larmor deprecates? The founders of the new electrical theory of matter have studied profoundly the laws of nature. They have made many permanent acquisitions to our knowledge; they have elaborated their theory with the greatest ingenuity, and yet the result has been to show that their theory is merely the same as the discarded ones, amplified and clothed in new names. The additional complexity, due to the desire to be more 
exact and more comprehensive, will do more than the criticisms of its adversaries to hasten the time when physicists will frankly avoid metaphysical explanations and start from experimental axioms.

In order to be exact when defining this new idea of the atom, I shall quote again from Ether and Matter: "The protion (or sub-atom) must therefore be in whole or in part a nucleus of intrinsic strain in the ether, a place at which the continuity of the medium has been broken and cemented together again (to use a crude but effective image) without accurately fitting the parts, so that there is a residual strain all round the place." So far this might almost be interpreted as the specification for a vortex atom; but, since such a type of strain fails to provide matter with electric charges, he diverges at this point and considers the " ultimate element of matter to be an electric charge or nucleus of permanent ethereal strain instead of a vortex ring."

When discussing these definitions, we should bear constantly in mind that the chief, if not the only, purpose of an atomic theory or of a mechanical model is to create a picture, however crude, of the constitution of matter. I can form absolutely no mental image of such a kind of matter as Professor Larmor proposes, and although I have discussed this new theory many times with its supporters, I have never found them able to give any clear and simple idea of such a strain; it 
is certainly unlike any that we have encountered in experimental mechanics or electricity. Let us strip the definition of technical intricacies, of such words as protions and ethereal electric strains, which have the power of confusing the mind and of making us accept statements we do not quite understand. In the first place, Professor Larmor's hypothesis is merely an evasion of the old atomic theory. His continuous, true matter which has absolutely no mechanical attributes and indeed none of any sort, except what he calls the ability to be modified by an electric charge, seems to me, as a substance, but very little removed from my idea of mere extent or abstract geometrical space. Sensible matter which consists of discontinuous variations of this true matter, not of a mechanical nature but of what he calls electrical strains, is to me less substantial than extent or space; for, as difficult as it may be, I can form some idea of space and I can communicate this idea to others, but I can make no mental picture of an electrical strain in a non-mechanical plenum; and such a strain certainly does not impress me as being matter as I know it. I shall now form these ideas into a chain: space is substance; matter is a variation or strain in substance; a strain is an electric charge; an electric charge is matter; matter is substance; therefore an electric charge and matter are both space, unless I can be persuaded that a variation of an entity can change its essence, which is absurd. This 
is a rather complicated syllogism, so it is better to reduce it to simpler terms, as follows: true matter is a plenum; by definition, a plenum is that which is full of matter; therefore matter is true matter. Such reasoning should convince anyone that, no matter how we may wrap up our logic, we cannot explain those things which we must use as a means of explaining other phenomena. The ordinary man expresses this by the saying, we cannot lift ourselves by our own bootstraps. Are not such hypotheses in the same class as perpetual motion and squaring the circle?

But even on the scientific side, this hypothesis is not satisfactory. Every theorist acknowledges that science is founded ultimately on our sense perceptions, and this fact should warn us that the attribute electricity is not a substance. The phrase, to electrify matter, has a well-defined meaning, because we know, by experience, that a body when rubbed exhibits a force which was not evident previously. We also know that we may select two different bodies, which will each show this electric force but of such opposite characteristics that their mere contact reduces the whole force to zero. Our knowledge of electricity comes only by this manifestation of electric force, so that if electricity were a substance we are reduced to this anomaly, that the addition of substance to substance may result in less substance. This attractive force is the simple and fundamental fact in regard to 
electricity and it is not proper to avoid it by speculating on matter in a state so special and so removed from common experience as when it is radio-active or highly vacuous. Now experience teaches us that matter added to matter is always more matter; thus satisfying our prime requisite for substance. This difference between electricity and matter is sufficient to explain why the term electrified matter is a real idea, and why materialized electricity means nothing. There is no more justification for calling electricity a substance than there is for doing the same thing for any other attribute of matter, such as color or temperature. The simple fact that matter appeals directly to our sense organs and electricity does not should be sufficient to convince anyone that our interpretation of nature, because of our material and mental organization, must be based for all time on a foundation of material substance and not of electrical substance. The postulate of the objective existence of matter is a necessary hypothesis and that of electricity is not. Is it to be supposed that, because certain learned men find difficulty in explaining some of the obscure actions of matter, the human race is to cast aside an instinctive and universally accepted axiom, any more readily than it will discard the idea that the straight line is the shortest distance between two points; that parallel lines never intersect; or that all bodies require three, and only three, dimensions in order to locate them;-because a few modern ge- 
ometers choose to advance other and contradictory postulates as axioms?

Let us now turn to some of the specific difficulties of this new theory. In addition to many absurd properties ascribed to the old elastic solid ether, its chief defects were, that it must, at the same time, have friction and not have friction, and that it could not account for electricity. If it had friction, then the ether would absorb light and heat energy, and the motion of bodies through it would affect the properties of light in a manner which could be detected; both of these have been found to be contrary to experience. On the other hand, the ether must have friction to permit the communication of atomic vibrations to it. To escape these dilemmas, Professor Larmor supposes the electro-magnetic ether to be without mechanical friction and so to remain quiescent when any body moves through it, but he apparently forgot that he had assumed that the essence of substance is electricity. If bodies are forms of electricity how can they move through an electrical medium without disturbing it? Since we have abandoned the theory that light and heat are mechanical waves for the supposition that they are electro-magnetic disturbances caused by periodic variations of electric charges, the new ether must, in some way, be modified by electric variations in order to receive and to transmit light and heat. But anyone can see we are just where we started. The electro- 
magnetic ether must have electro-magnetic friction, to receive the electro-magnetic vibrations of the electric atom and must be devoid of electro-magnetic friction so as not to absorb electro-magnetic waves when in transit. Again, he says the mechanical atom was defective, in that it could not account for electricity, but he also says that the electrical atom does not explain cohesion, weight, and, I might add, any other mechanical property. Which shall we choose? Lastly, not to prolong the discussion, just as the mechanical ether and atom led us to such contradictory attributes as enormous rigidity and inappreciable density, so the electro-magnetic ether and atom require us to give the latter an electric force whose magnitude is to that of weight as ten raised to the forty-second power is to one.

Temperamentally, Professor Thomson has little in common with his colleague, Professor Larmor; as all know, we owe to him a long series of most delicate and profound experimental investigations in this field. Like Lord Kelvin, he is usually content to construct a model for each special phenomenon, and, as he is rather indifferent whether these agree in operation, it is difficult to form any consistent idea of his theory. He seems to regard matter as if it were a sort of buildingblocks which may be put together as fancy directs. His best-known conception of matter and electricity is his picture of an atom as a central sphere of uniformly 
distributed positive electricity with points of negative electricity so placed in it as to form the corners of regular geometrical figures. He then calculates the stability of each figure from the mutual attractions and repulsions of the charges and supposes the chemical elements to be conditioned by this stability. The scheme is so frankly artificial and speculative that its author can regard it only as an architect does his sketch, as a symbol of a house. But in addition to his pictorial representations of matter and phenomena, which, if taken correctly, do not deceive anyone, he subscribes to the hypothetical method and is a leader in developing the electrical theory of matter.

If we now turn to a consideration of the ideas of Professor Lorentz, we shall find a close agreement with the hypothesis of Professor Larmor. We have been led to the conception of electrons or protions, Professor Lorentz says in his Theory of Electrons, by our desire to understand the electrical properties of matter. For, while we adopt the theory of Faraday and Maxwell that the space or electro-magnetic ether near an electrically charged body is put in a certain state of strain and never lose sight of this idea, yet we need not form an image of it and really cannot say much about it. In fact, these strains, as imagined by them, cannot have a real existence without producing motion in the ether, which is an impossible assumption. On account of the difficulties into which such speculations 
lead us, there has been a tendency of late years to avoid them altogether. But while this method is the safer one and provides us with a set of correct formulæ and laws, yet we are compelled to introduce into these equations unknown terms, called coefficients, which express the individual properties of different kinds of ponderable bodies. For example, the same magnetic force produces different magnetic effects in iron and copper. If we wish to obtain a deeper insight into the properties of matter, he says, we must not be satisfied with simply introducing for each substance its special coefficient, whose value is to be determined by experiment; we must invent some hypothesis about the mechanism of matter which causes such differences of behavior. It is this necessity which has led us to the hypothesis of the electron. He then defines these electrons as extremely small particles, charged with electricity, and present in immense numbers in all ponderable bodies. They are of two kinds, positive and negative, and are free to move in conductors of electricity and bound to points of equilibrium in non-conductors. Sometimes he considers them to be rigid, and at other times as deformable bodies. Their inertia, or mass, is, for the most part, an effect of their electric charge, and the negative electron is probably free electricity without ponderable mass. Professor Lorentz assigns no specific properties to the ether, but he is required to assume that it can penetrate freely all parts of the 
electron. It is evident that the philosophical ideas of this theory are the same as those previously discussed. We are driven in both to the supposition that the electron is a space modification of a universal medium and that, in some way, neither electron nor ether is a material substance, but a kind of transcendental entity called electricity.

Of course we wish to obtain a deeper insight into the properties of matter, and if we had discovered, or had any means of discovering, a method to such a knowledge, no amount of labor would be superfluous while making an hypothesis. But we wish many impossible things; we should like to be satisfied about the immortality of the soul; the causes and nature of life, and a host of other problems. Now Professor Lorentz says we know very little about the ether and the nature of matter, and by very little, he means we know nothing. What advantage, then, does he propose, when he says: Come, let us invent something about these unknowable things, let us make an hypothesis that we may seem to obtain a deeper insight into the mysteries of nature? He knows that the atom of iron is as mysterious as a ton of that substance, and that he does nothing to explain the nature of iron by giving imaginary properties to a sub-atom of iron; he is aware that he has not touched the reason why iron differs from copper, and he ought to know that, by calling this subatom a particle of electricity, he has only added con- 
fusion to our ignorance. What would he say of a biologist who, growing tired of describing forms of life which he can handle, gave the habits and appearance of marine animals living beyond the reach of his dredging nets? The security of experimental science rests on the belief that, for the honor of science, no one will tamper with what he has actually observed. Why should more leniency be granted the theorist, why should he be allowed to mix fact and fancy, law and hypothesis, as he chooses, and not be called to account? It is just this restraint, this distinguishing between what we know and what we desire, that constitutes the value of science as a discipline of the intellect ; and in the exercise of this restraint lies our intellectual integrity. The gospel of science, for it has one as truly as has religion, may be summed up in the words of Goethe: " in restraint first shows himself the master." What has it come to, when we can pass over such false methods with a shrug? Huxley spent his life, in season and out of season, waging battle with bishops and laymen to convince them that religious belief and religious theory must square with objective fact. And he assured them that men of science could provide a foundation of truth, and would not impose a false one, so that all men might know and have a rational basis of life and belief. And how have we kept the faith? We have given with equal emphasis our speculations and our observations. Nor can Professor 
Lorentz say he has kept in the certain path because he has warned us in the beginning of his treatise that he is dealing with glittering hypothesis. He takes all the force out of the warning by using his genius to overlay his speculations with a specious appearance of reality. Let him try the opposite plan and actually convince a class of students and educated men generally that his hypothesis does not, and cannot, give any real insight into the actual properties of matter, that he is talking in a Pickwickian sense, and how much serious attention would he attract? No, the world still believes in the restraint of men of science and in their boast that they will submit our vague longings to the test of experience. And it will be a bad day for science if this belief is destroyed.

Nor is it proper to adopt an attitude of indifference to these hypotheses as many experimentalists do, to pass them by with a shrug or with the statement that they cause little harm to the growth of science, however little they may aid it. If science were merely a mental gymnastics, this indifference would be well enough, but science is increasingly considered to be a guide to conduct. We have no right to approve the intellectual carelessness which has been so vividly expressed by $\mathbf{M}$. Poincaré in the preface to his Théorie de la Lumière: "It matters little to us whether the ether really exists; that is the business of the metaphysician to find out; the essential thing for us is that 
everything acts as if it existed [?] and that this hypothesis is convenient in explaining phenomena. After all, have we any other reason for believing in the existence of material objects? Is not that belief also a convenient hypothesis; only we shall never cease to make it, meanwhile the time will come, without doubt, when the ether will be rejected as useless." Both material objects and the ether may be only hypotheses, or rather our knowledge of them is relative, but not in the same way; the existence of material objects is based on direct experience and the existence of the ether is not. However we may argue, the objective reality of matter is a necessary idea, fixed in our minds and not to be dislodged.

Criticism is now often directed against the older atomic theory because a given form of it no longer accords with phenomena which have been lately discovered. And it should be borne in mind that this new theory of electrons has been developed with the main purpose of supporting the atomic theory and making it agree with our new knowledge of electricity. Thus Professor Rutherford has recently performed a beautiful experiment by which he detects electrically a portion of helium gas, which he calculates to be of the dimensions of a chemical atom. Here, he says, we have at last an actual experimental proof of the reality of the chemical atom. Does not this experiment show just the reverse? The idea underlying all atomic 
theories is that the indivisible unit of matter is so small that it can be dealt with experimentally and mathematically in aggregates only. So when he devises an apparatus so delicate as to detect the action of a single particle the size of the so-called chemical atom, he forces us to adopt for the real atom a smaller unit whose individual variations will be beneath our observation. The unit of matter becomes just one degree further removed from matter as we know it. Instead of squaring our hypotheses with the sensible properties of matter, we may thus more easily make matter a purely transcendental quantity which we create according to our own imaginations. Would it not be better frankly to say the material universe is merely a world of ideas, an embodiment of intangible motion, energy, and electricity, rather than to keep up the fiction that the electron is the ultimate unit of substance?

Apparently the chemical molecule is a well-defined point in the regular divisibility of matter where certain physical apparatus, as the balance, fail to record variations in so small a body; but, by the use of chemical appliances, we are able to take note of still smaller masses, which have been named the atoms of the chemical molecules. At this point these methods become too gross, and we next have recourse to the electrification of the gaseous atoms by the X-rays or by radium, and can then detect variations in these 
particles by means of the electroscope. These smaller portions of matter are called electrons or sub-atoms, and for the time being we have rested here. But would anyone say that new methods of analysis and new apparatus of registration are an impossibility, and that the electron will not in future be divided? On the other side of the series the mote, dancing in the sunbeam and disappearing when the light fails it, is an indivisible atom to the unskilled man deprived of sensitive apparatus. The fact is, the atom as an objective unit of matter has no existence; we name that portion of matter an atom when we have reached a limit of appreciation of matter by our most sensitive apparatus.

If these fundamental and irrational assumptions of plenum and sub-atom be once granted, then a mechanical explanation of many of the phenomena of nature follows logically. But this is also true if we postulate that matter is composed of the four essences,-earth, air, fire, and water; and one has only to recall the success of Descartes with his three elements. The question is, are the postulates true? If we cannot verify them by experiment, then science should reject them. On this criterion the sub-atom is as vulnerable as any other postulate. The conclusions, deduced, may give a truly mechanical explanation; for, in spite of denying the existence of matter in the beginning, the substitute electricity is at once endowed with all the essential characteristics of the discarded 
matter, such as inertia, conservation, gravitational attraction, extent, etc. Certain additional properties add to its conquests, since, by splitting up the atoms, a new set of pawns is available to the players of this game of probability and chance.

The diversity of the chemical elements may be said to result from the various stable combinations which the sub-atoms may be made to assume. Professor Rutherford developed his ingenious theory of radioactivity by supposing certain elements to be in a state of comparatively unstable equilibrium. A definite proportion of their atoms explodes continuously, reducing the atomic weight of the parent body, and supplying the spontaneous energy noted experimentally. This process continues until a stable form is reached. But does he not forget that one of the triumphs of the old atomic theory, a proof that the chemical atom could never be divided into sub-atoms, was that when an unstable compound, like gunpowder, exploded, the constituents were found to be stable chemical atoms?

The phenomena of electricity are naturally the main problems attacked. A current of electricity in this new hypothesis becomes the flow of immaterial particles of electricity in a metal conduit, carrying with them their energy of motion. In non-conductors, like glass, they may be heaped up into an excess of positive or negative to provide a picture of the free charges on such substances. Radiant light, heat, and electricity are 
the periodic disturbances produced in the plenum by the oscillatory motions of the same particles about fixed centers. These are said to be useful ideas and clear explanations. But are they? Have we accomplished anything more than to reaffirm the statement that a current of electricity under certain conditions flows through a wire, when we say a stream of charged particles moves through the wire? In the first place, we create the particles, and next endow them with an occult power of motion. Again, when zinc and copper are placed in contact and separated, the zinc becomes positively charged with electricity, the copper with negative, and the two attract each other. Do we learn anything more when we affirm that an excess of positive electrical particles passes into the zinc and negative ones into the copper? Why should they act so? In both cases we have merely stated an unknowable cause in different words. The law remains the same whether we say electricity or electrical particle, and the former term expresses less pretense of knowledge.

Far more significant, and less justifiable even, is the attempt to explain the mass of a body as an attribute of electricity. Mathematical analysis shows that an electrically charged body, moving with great velocity, has a resistance to motion apparently greater than when not so charged. Now, they say, continually diminish the ponderable mass of the body and maintain the electric charge constant; the electro-magnetic mass, as this 
effect of electricity is called, becomes proportionately greater and greater. Continue this process indefinitely, and at last all the matter is gone, and there remains a free electric charge, an immaterial point of electricity, moving and possessing inertia or mass. And just here lies the greatest danger of all such hypothesis-a total confusion of fact and fancy. In the first place, it is not even based on experience, since the most rapidly moving bodies of a sensible size have a velocity far too little to make this effect noticeable. But, what is more important, it is a method of thought to be used with the utmost caution even in experimental processes. The warning against such extra-polation is metaphorically displayed in every physical laboratory. How we would scorn the unscientific conclusions of the metaphysicist who reasoned in the same way! Let us imagine one saying that goodness is an attribute of small, as well as of large men; now diminish the size of a man and let his quality of goodness remain constant, then we shall have an entity, concrete goodness, left when the man shrinks to nothing. It is not necessary to use such an absurd example, as a parallel case exists in physical phenomena. A sphere, moving in a fluid, experiences a like increase in apparent mass, due to the necessity of imparting energy to the fluid. Now decrease continually the density or mass of the body and maintain its volume and velocity constant; the hydrodynamic mass becomes proportionately 
greater. Continue this process until the matter is all gone, and there is left a sphere of something with an apparent mass still moving through the fluid. Will it not be difficult to persuade anyone that the something moving did not vanish simultaneously with the material sphere-that the attribute did not vanish with the entity? It will be just as hard to convince the future scientist, when the vogue of the electrical theory departs, that an electrical charge remains after the electrified matter is reasoned away. Such ideas leave us in the same foolish state as the hunters of the Snark, who, after incredible labors, came to the place where a Snark should be, and found it was a Boojum which vanished silently away.

If $I$ am correct in believing the fallacy to lie in trying to explain natural laws, it is not pertinent to inquire further into the working of this electrical hypothesis. The essential point is whether physics has anything to do with the nature of matter and electricity. Atoms and ethers of any kind are metaphysical creations; the mechanical models built on such an unsubstantial foundation require a god to set them going, and are, at best, an ineffectual means of describing phenomena previously observed, and not finger-posts to new discoveries.

The claim, that such arguments as this are ineffectual criticism because they tend to destroy the scientific method most used and offer nothing better in its stead, 
is frequently made by scientific theorists who recognize the artificiality of their hypotheses but who feel that, in some way, they tend to good. They forget that the growth of science has always been largely due to the discovery and the elimination of error. If we wish to make a garden, the first necessity is to clear away the weeds, and the same is true of science; it is as important to know what is wrong as what is right. But even this claim is not correct, the better method is known and is in use. Have they forgotten the work of Galileo, of Newton, of Franklin, and of many others, who employed the abstractive method, or, at least, kept their hypotheses well in the background? Nor is it difficult, if one studies the history of physics, to outline the basis of a method in harmony with my ideas, as I shall do in a later chapter.

Nothing has been more convincing to me of the advantages of the abstractive over the hypothetical method than a study of Descartes's Principia Naturae. Modern theorists have acknowledged their debt to him; how great is this obligation can be seen from the discussion of Descartes's system which follows. A comparison of his postulates and conclusions with those of our modern theory shows them to be almost identical, if we change his antiquated knowledge and his discarded metaphysical language into modern terminology. And lastly we should compare Descartes with modern physicists; his knowledge of the phenom- 
68 THE LIMITATIONS OF SCIENCE

ena and laws of nature is insignificant, so great has been our progress in this respect, but as a metaphysicist he is still the master of modern scientific hypothesis, so little has been accomplished in that field in two hundred years. 


\section{CHAPTER III}

\section{DESCARTES AND HIS INFLUENCE ON RECENT SCIENCE}

De s'égarer avec méthode.-Michinet.

If it be true that the permanent advances made in science have been the result of observing phenomena and of classifying them in laws which are as often as possible to be expressed by mathematical formulæ; and if it be also true that we have persisted in the effort to explain the causes of phenomena without really advancing knowledge; then it will be most useful to compare our present state with that of an earlier time. For this purpose, I have chosen to sketch the scientific system of Descartes. In the first place, there can be no doubt as to the enormous increase in our positive knowledge of phenomena and natural laws since his time; the difference is so great that there is no need to dwell on it. And yet, if we consider the history of Descartes's hypothetical cosmogony, we learn that it was discarded only to have a revival at the present time. The advantage of reviewing the system of Descartes is all the greater because it seems apparent that many of those who are quoting his authority have not an accurate knowledge of what he taught. 
So far as I can see the hypothetical method has its most illustrious example in Descartes, and instead of aiding us to gain real and clear ideas, he has burdened science with useless and complicated metaphysical systems. Instead of being a symptom of power, the reluctance to recognize the limits of science comes rather from a certain intellectual cowardice which refuses to acknowledge the truth, that we cannot attain any knowledge of things themselves but only of their attributes as they affect our senses. If we really face the question; strip our scientific hypotheses of their technical phraseology and complex logic, and try to get a clear and simple idea of what they mean, we find that we have been deceiving ourselves. In the first place, we use words, which ordinarily convey definite ideas, in a sense purely symbolical, and then confuse the image and the reality. For example, when we define space or the ether as a perfect fluid, we deceive ourselves into believing that we have gained a clearer idea of space by applying to it a term which signifies the mobility of matter. But this attribute can be applied only to a material fluid whose change of position may be measured, and such a fluid appeals to us as something essentially different from immaterial space. Nor do we overcome this difficulty by qualifying space as a perfect fluid; any fluid is perfect which satisfies the laws of its nature, and we practice deception when we inject the ethical meaning of perfect, as being some- 
thing above ordinary criticism, into the scientific definition of a fluid. We have also constructed a symbolic language, called mathematical analysis, whose characters and terms are so removed from ordinary speech that it imposes on our minds an impression of not being limited by the bounds of logic. Thus, if we derive a mathematical formula for the quantity of heat or electrical energy which passes through free space, we deceive ourselves by thinking that we have an expression not only for a quantity of energy, but that also we have in some unaccountable way gained a knowledge of the nature of energy and of the attributes of space. And we slur over the scientific axiom, that since these mathematical symbols did not express in the beginning something concrete, they cannot after any manipulation give a result which is other than imaginative. Again, we postulate some entity such as matter, energy, or electricity, as a foundation and attempt to derive logically from it all the phenomena of nature, and ignore the plain fact that nature, as a whole, reveals itself to us as a succession of events, either not connected at all in a logical sequence of cause and effect, or at least in such an intricate tangle as to defy our powers of analysis.

During the last two decades, there have been discovered an unusually large number of physical phenomena, and it is no exaggeration to say that we have mastered them with surprising rapidity and with great 
ingenuity. At the same time we have turned to these new manifestations of matter and energy with the hope that in them we have at last found the materials for a new and lasting scientific cosmogony. But the edifice differs in appearance only from that built long ago by Descartes; and the materials in both are the same, changed in name but not in substance.

By a scientific cosmogony, as distinguished from revelation and from metaphysics, is here meant that we first postulate an archetypal form of substance and certain fundamental forces, few in number and inherent in this entity, and that then the universe, as it now exists, follows as the result of the continued action of these forces on this substance. That is, the state of the universe may be expressed at any time subsequent to the initial action of the forces by a set of mathematical or verbal formulæ. And if we could actually, as we can theoretically, reverse the action of these forces in time and in direction, the primal condition of the universe would again result. It is furthermore postulated that this archetypal substance existed originally in the form of minute particles, separate from each other and exactly similar in character, and that the forces acting on these particles were in such perfect balance that the total value of their effect was zero. From some cause, generally unexplained, this balance was destroyed and, like a clock 
when its pendulum has once been jogged, the panorama of cosmical history unrolls itself inexorably. Thus the universe becomes a sort of machine whose parts are mechanically driven, not by some external motive force, but by the mutual reactions of its parts, and so destined in time to run down when this internal and available energy shall have exhausted itself. And when we speak of the universe, all that part of it designated as living bodies and vital forces is to be included, because of the belief that they also can be considered as ponderable masses subject to physical and chemical forces.

As an original hypothesis the work of Lucretius is of no consequence. In fact his atomic theory was taken almost entirely from Democritus, and it was rather by the vision of the poet than by the logical analysis of the man of science that he developed these ideas into a picture of the nature of our world. His theory was, in brief, that the universe contained in an otherwise empty space an indefinite number of indivisible and immeasurably small particles, called atoms, which differed only in size, position, and shape. These atoms were indestructible and by their combination and separation formed all natural bodies. The motion of the atoms did not arise from external forces but was an inherent property of their nature. With a common impulse, they all moved toward the center of the universe, but in addition they possessed an individual 


\section{THE LIMITATIONS OF SCIENCE}

power of irregular deflexion which introduced variety in matter. By their union they formed bodies; and by their impact and rebound they caused vortical motions which now find expression in our term, energy. As an explanation of natural phenomena the theory has no value, as it originated long before the mathematical laws of forces had been formulated or the phenomena of matter had been accurately observed; but as a guide to thought it has been the basis of most of the later scientific theories. Gradually elaborated by Gassendi, Newton, Boyle, Kant, Laplace, Dalton, and others, this atomic theory is still the touchstone of modern chemistry and physics.

Possibly the vastest consequence of the atomic theory is the nebular hypothesis developed independently by Kant and Laplace. Essentially this theory is a restatement of the ideas of Democritus and Lucretius, yet the discovery by Newton of the mathematical law of the force of gravitation permitted for the first time a true scientific method. Thus, while they were compelled to assume, as arbitrarily as did Lucretius, an initial state of chaos when matter was scattered in atomic masses throughout space, they nevertheless had a cause when for any reason the original balance of forces was disturbed, experimentally verified and mathematically expressed, to account for the gradual agglomeration of atoms into larger masses at definite places, from the fact that any two masses of sensible 
size were known to have a mutual attraction varying inversely as the square of the distance between their centers. The active attribute of matter, ruling and guiding its motion, thus became independent of fancy and subject to experimental verification. The nebular hypothesis has remained more or less a fragment, limited to the determination of the sizes, masses, and positions of celestial bodies and systems. Neither Kant nor Laplace, except for a discussion of temperatures, included in their scheme of the evolution of inorganic matter the causes of the complex forms and forces of matter now observed on the earth or stars. Nor beyond assuming that matter was determined by mass and an inherent power of attraction, did they make any attempt to explain how this occult force acted through a distance. And from this simplified idea of matter without variety and without complexity, they were able to deduce mathematically and logically the idea of the evolution of cosmical matter, from a condition of primitive distribution, at least to its segregation into cosmical systems: suns, planets, and satellites. And if we remember that Kant approached the problem from the standpoint of the metaphysician and Laplace from that of the pure mathematician, we can readily understand why they both were satisfied to take such an abstract view of the universe, and also the simplicity and the insufficiency of such a method. Astronomy is the one science, because of the comparatively enormous 
magnitude of its units, which permits such an abstract simplification of matter as to consider it merely in the form of masses concentrated at mathematical points. Where we deal with bodies measured in millions of tons and with distances in millions of miles, such variations as are found in the laboratory sink into comparative insignificance and we can deal with averages instead of particulars. On the whole their attempt was a success since we can find few flaws in the general conception of a nebular hypothesis, however we may differ as to details. And just because the phenomena discussed are so few in number and so general in character, there was not the need for supposititious causes and fictitious attributes, provided we grant their initial state of the universe and the first cause contributing to irregularity and the beginning of motion. These postulates are of course entirely arbitrary and require us to assume an existence previous to time, since time has no meaning in a universe which is everywhere in a state of perfect equilibrium and rest. Undoubtedly the reason why this hypothesis persists in its principal features to the present time, in spite of constant attacks, is just because its authors exercised selfrestraint, holding to general laws and avoiding specific details. Some form of cosmical evolution is now generally granted and the heavens are swept by gigantic telescopes which linger on the nebulæ, in the hope that accurate measurement will show that these apparently 
chaotic masses are slowly changing into the more stable form of stellar systems.

Side by side with the belief in atoms, separated from each other in empty space, had grown up the directly contrary idea that substance is continuous and space is a plenum. This doctrine permits us to make no essential difference between space and matter. Space becomes a continuous substance unvaried, and so imperceptible to our senses, while matter is merely a localized variation of this same substance of such a nature as to make it perceptible. And it is significant of these two systems, that although they begin with contradictory premises, they both lead to the same conclusions after apparently rigorous deductions.

The doctrine of continuity owes its rise to Heraclitus and, amongst the Greeks, was most highly developed by Aristotle. They announced this postulate because the notion that a body can attract another through an intervening vacuum and cause it to move introduces an occult or inexplicable idea. Our experience teaches us that motion results only from a push or impact between bodies actually in contact. But it is quite evident, that in refusing to accept a force which acts contrary to our experience, which all goes to show that a material link is necessary to produce motion between bodies, they fell into as serious a difficulty, for they were compelled to create an occult substance to serve as the material link between bodies, and occult 
variations in it for the bodies themselves. As explanations of phenomena both ideas are occult, but not to the same degree. We know, for a fact, that there is an attractive force between bodies of sensible size, although we cannot explain its cause, and we can in our imaginations transfer a like form to bodies of an insensible size with some probability of truth. But the postulation of a plenum of continuous substance and of variations in it, which affect us as matter, is wholly occult since we have no experience from sensible matter to guide us; in fact, both plenum and its variations always have characteristics assigned to them directly contrary to the evidence of our senses.

The history of scientific theory is a record of the conflict between these rival ideas of continuity and discontinuity of substance. While the atomistic school has frequently had the advantage, since the time of Descartes the doctrine of the continuity of matter has persisted in some form, and at the present time is again established as the basis of physical theory. Nevertheless, the cosmical system as deduced by Descartes from this hypothesis of a plenum has suffered shipwreck and comparatively few think it profitable to study it in detail. Before we adopt his principles and discard his conclusions, it is at least advisable to see whether his errors lie in the principles themselves or in the inability of Descartes to derive true conclusions from correct principles. Fortunately, we have in the 
Discours de la Méthode, in the Principia Naturae, and in the many letters of Descartes an unusually complete record of his principles, his method, and his conclusions.

In character, training, and opportunities, few if any philosophers have been better equipped for their task than Descartes. As has been said of him, "he is a type of that spirit of science to which erudition and all the heritage of the past seem but elegant trifling." $\mathrm{He}$ believed and acted on the opinion that no scientific knowledge is attainable unless men doubt; unless they put aside authority and rely on their own experience.

Descartes has in his Principia Naturae set forth with specious simplicity the causes, laws, and the phenomena of the universe as he finds them. Geometry is to be the ruler or at least the vicegerent, and no substance will be discussed except such as may be divided, figured, and moved according to the laws which geometers hold to govern quantity, nor will any proposition be considered proved unless it has been deduced with such evidence as would suffice for a mathematical demonstration. With vexatious inconsistency, he then destroys the force of this admirable introduction by carefully warning us not to consider his premises true or his conclusions conformable to fact, since his scheme is really an hypothesis or supposition as to what might be and not what is. This caution is usually attributed to his fear lest he should 
arouse the hostility of the Church and suffer a penalty similar to that which had just overwhelmed Galileo. His own plea was that he valued peace more than the spread of his opinions and also that he had always been a good Catholic and bowed to the authority of the Church, even when its decrees were contrary to his reason. But it is also possible that doubts arose in his own mind as his system developed and that when he found it necessary to compromise with the rigorous principles he had announced, he tried to disarm criticism by the plea that after all he was really concerned only with a fictitious world.

At all events, while the laws of the heavens were under consideration, he permitted this veil of unreality to remain very thin; for he knew the Church wisely allowed considerable latitude of thought concerning those regions of space which apparently contained no subjects to Catholicism, and he felt scientifically safe in a field where verification by experiment was only beginning to be advocated. But the case was altered when he came to discuss terrestrial laws and phenomena, for here both Church and experience held sway and must be conciliated. In this field Descartes, mindful probably of both of these perils, relied on the same hypothesis, but to placate the Church he insisted that it was to be understood only in a most figurative sense, because we know that God created the earth, finished and perfect, as revealed in the Mosaic account; 
and to disarm scientific criticism, his postulates he declared to be fictions, but in some way without departing from correct mathematical deductions, his conclusions were similar to experience.

It would be difficult to understand from these contradictory statements what was Descartes's real opinion of his scheme. But he has this answer in another part of his Principia, an answer which carries conviction. Since we have an idea of perfection, we have also the certainty of the existence of God, or an all-perfect Being. And because this God is justice itself, if the principles laid down by him are evident, if the consequences are founded on mathematical laws, and if the deductions agree with experience, it would be ascribing injustice to God to believe that the causes of the effects are false. Now observe how far Descartes believes he has fulfilled these conditions: " je ne pense pas qu'on doive recevoir d'autres principes en la Physique, ni mesme qu'on ait raison d'en souhaiter d'autres, que ceux qui sont icy expliquez."

Descartes has recorded for us how he arrived at the postulates from which he developed his natural laws. Having previously laid aside preconceived ideas, he found that to doubt is the first and only means of knowledge; we can doubt the existence of everything except that which doubts, therefore that which doubts or thinks, exists. Whence he derives the principle that our thoughts and the things we have an idea of, 
are real and objective to us. However, by such a process we do not obtain a knowledge of things themselves, but only of their attributes. Hence, the final reality must be those attributes which are inseparable from our conception of all phenomena, and there should be some one essential attribute which may be used to designate things in general. Thus hardness, while it is an attribute, is not essential, for a body moving at the same speed as ourselves does not give us the sensation of hardness. After careful consideration he found that simple extent in length, breadth, and thickness is the one attribute common and essential to all matter. Not only is this true, but the extent of matter is identical with the extent of space: "The same extent in length, breadth, and thickness, which constitutes space, constitutes a body; and the difference between them lies in this, that we attribute to a body a particular and limited extent which changes position with the body as it moves, and that we attribute to space an extent so general and so vague that when we remove from a certain space the body which occupied it we do not think we have transported the extent with it; meanwhile the extent of the body remains of the same size, of the same figure, and has changed position with respect to the body only as we determine position by other bodies."

The reason, according to Descartes, why we have been led to believe that substance is different from 
space, and that it consists of atoms either in a vacuum or in a non-material medium, is the property known as expansion and contraction. As an illustration, he instances a sponge, which changes size but not character when its pores are filled with water. Here we have a change of size from the change of position of spongematter, the two kinds being of the same essence. So the change of volume of any body is due to a change of position of matter-substance produced by a change of space-substance, which also are essentially the same and differ only in accidental qualities.

Furthermore, since we cannot imagine a space so great that there cannot be a greater, or so small that it cannot be divided, then as matter is essentially the same as space, it too must be indefinitely great in extent and indefinitely divisible.

This is a very complicated way of getting back to one's starting point. He says first, that substance is extension and then that space is extension, but since extension is everywhere and space is nowhere vacuous, a body is a body.

Having discussed the nature of matter in general, he then finds that diversity in matter and also its differentiation from space can consist only in variations of position and motion of small parts of space which are themselves exactly similar. As corollaries to this definition, it follows that there can be neither absolute position nor absolute motion. To determine the posi- 
tion and motion of a body we must use some other body as a point of reference. But in this system, all space is capable of motion and, in fact, is in rapid motion, and with no possible stationary reference point all positions and motions are merely relative. This question of absolute motion has been the subject of much discussion in the last few years, as certain phenomena of light require that, granting a luminiferous ether, it must be absolutely stationary. And experiments have been devised to determine whether we can measure the absolute velocity of matter with respect to the ether. As in all experiments involving the ether, the results are nugatory.

Nor, in the theory of Descartes, can there be action at a distance, for matter is continuous and all motion is the result of a push or impact. Motion, therefore, he defines as the transference of a part of matter or of a body from the neighborhood of those which touch it immediately, and which we consider at rest, to the neighborhood of others. And since all space is full of matter, or rather is matter, each body is so fashioned that it can never occupy a greater or a less space, nor can any other body occupy the space while it is there; therefore if a body move to another position, it must displace an occupant; and this, another; so that every motion results in a closed ring of moved bodies, each finally occupying the space left by its previous occupant. 
These postulates of Descartes, that space is a plenum and motion the result of an impact, required him to oppose such theorists as Gassendi who were advocating the atomic theory and an occult attractive force in matter as its cause of motion. It thus became of prime importance for him to formulate laws of impact and motion. This was an extremely difficult problem, especially so as the available knowledge of the phenomena of impact and motion was very deficient and, such as it was, indicated that friction produced an uncompensated diminution of velocity. With great care and in minute detail, he announced such laws as would, in his opinion, maintain constant the total quantity of motion or momentum of the universe in spite of friction. That is, such laws as would transfer the loss of motion of one body by impact to an equivalent increase of another. These laws are:

God in his omnipotence created matter with motion and rest.

If a body move twice as fast as another twice as great, the quantity of motion of the two is the same.

All bodies, which move, tend to move in straight lines and can change their direction only by encountering others.

If a moving body strike another of greater momentum (plus fort) than itself, it loses none of its motion and if it strikes another with a less momentum 
(plus foible) which it can move, it loses as much motion as it gives to the other.

Then seven other laws of impact of less importance follow, which it is not necessary to quote, as Huygens later showed that all eight were, for the most part, erroneous.

In spite of the fact that Descartes, without verification, invented the laws of motion of bodies and of their impact, yet we must recognize that he sought and found one of the great scientific principles; that unless the universe is tending to a state of uniform rest through dissipation of motion by friction, some active property of matter must be conservative in addition to its quantity. While his particular law of the conservation of momentum was erroneous, yet it is undoubtedly the progenitor of the law finally enunciated by von Helmholtz and now generally accepted, that the total quantity of energy remains constant.

With these general principles settled, the nature of space and matter, and its conservation of quantity and action, we shall defer criticism and describe the various kinds and phenomena of matter as nearly as possible in the sense of Descartes.

We are to suppose that, in the beginning of time, God divided all space or substance into equal parts, which, contrary to the chaos pictured by poets and philosophers, had been previously in perfect uniformity and rest. Out of these primordial particles of space 
were fashioned all the small constituent parts of matter which now compose not only all terrestrial and celestial bodies but also the interstellar spaces. While we do not know the actual size of these particles, as they were originally or as they are now after they have suffered changes, some growing smaller by division and others larger by the accretion of many into one from causes which will be described later; yet their first size must have been the exact average of all the constituent particles now comprising the universe. God also caused these primordial particles to move, and with equal force in two diverse fashions: each to rotate about its own center to form the constituent elements of the interstellar spaces, or, as Descartes calls them, the fluid body of the heavens, and many to revolve together about common axes, creating vortices whose centers are the nuclei of the sun and fixed stars in the heavens. The velocity of this uniform motion was the average of all the diverse motions existing now, so that the quantity of motion or momentum has remained constant throughout all time.

Although these primordial particles were of equal size and motion, they could not have been spherical in shape, since a pile of spheres does not fill a space and there must be no vacuum. But whatever their initial figure may have been, they have in the course of time become exact spheres. The cause of this change of shape is, that the force, by which they were first moved, 
was sufficiently great to separate them from each other, and was therefore great enough to knock off their angular edges and corners because of the concussions of their future impacts. The result of such a constant attrition is well known to be that the body approaches the figure of a sphere, just as the pebble on the seashore becomes round from the grinding of the waves and its fellows.

This action gives rise to three forms of matter which constitute the universe, and whose various forms of aggregation and proportions of mixture, with their resulting varieties of motion, account for all the diversified kinds and attributes of matter and space.

The first kind of matter is the cosmic dust of attrition or the little fragments resulting from the above mentioned collisions. They are excessively small and have acquired enormous velocities, because the larger particles, from which they were split off, have constrained them to move faster than themselves through the narrow and tortuous paths formed by their interstices, just as air in a whistle moves the faster, the smaller the orifice from which it issues. They are also so numerous, so various in size, and so irregular of shape, that there are always sufficient of them ready to hand to fill exactly any space which might otherwise be left vacuous by the arrangement and motion of their parent sphericules.

The second elementary kind includes all the rest of 
matter and space, whose least parts are spheres and smaller than any body we know, but they are much larger than matter of the first kind.

There is also a third kind of matter formed by conglomerate masses of the other two kinds, which have become so linked and interlaced together as to be inseparable, at least by human means. The pores of these masses are filled with the spheres of the second kind and the fragments of the first kind.

The first kind of matter forms the sun and the fixed stars; the second kind is the material for the so-called empty spaces of the heavens; and the third is the basis of the diversified bodies composing the earth, the planets, and the comets.

As stated before, this elementary matter was originally endowed with two kinds of motion: the rotation of each particle about its own axis and the revolution of many about a common axis, forming a vortex or whirlpool. Descartes then portions out the heavens so that the axis of a vortex will pass through the center of each star, including the sun. The outer limit of each vortex extends so far in every direction as to touch the confines of each of the neighboring starvortices; in this manner all space is occupied by them, rushing about their several axes with a velocity decreasing as the distance from the axis increases. It is naturally a problem of great intricacy to place these immense vortices so that they will not interfere with 


\section{THE LIMITATIONS OF SCIENCE}

each other's motion, and he spends much labor and great ingenuity in describing the possible inclinations of axes and directions of motion of such a system, but not satisfactorily.

The sun and stars, as they now are, were not created contemporaneously with the vortices but were a gradual growth from them. When more dust of attrition was ground from the rotating matter of a vortex than was sufficient to fill the interstices of this matter, it began to flow toward the center and along the axis of the vortex, gradually forming there a nucleus, très subtil et très liquide; namely, a luminous star. According to Descartes's idea, which in spite of his contempt for authority he borrowed from Aristotle and the schoolmen, these stellar masses, consisting of matter of the first kind exclusively, are pure celestial fire, which is thus an entity and of all things the most fluid and so violently agitated as to be able to disintegrate the most solid bodies. Fire of itself does not need to be replenished but only seems to need replenishment, because the particles of a terrestrial fire are constantly flying away and being dissipated, and so must be renewed by others derived from the pores of gross combustible bodies and of air; but in celestial fires there is no need of external replenishment because the steady stream of fire-substance, flowing of its own accord to the center of the vortex, maintains a constant supply. Alas, that the spectroscope should show us that our sun 
and the stars are not this pure elemental fire but merely heated matter of a terrestrial nature. Such havoc does experiment play with a grandiose hypothesis. We may, however, pass over difficulties impossible to have been foreseen by Descartes, since many men of science still think an hypothesis valuable if it escapes contemporaneous troubles, and of these there are always an abundance.

Between these star centers lie the great interstellar spaces which some believe to be vacuous but which Descartes supposes are packed with matter of the second kind. The interstices, which would otherwise exist between these little spheres, are filled with matter of the first kind. And all together they rush around common axes to form the vast heavenly vortices with a velocity enormous but less than that of the celestial fire of the stars.

This accounts for all the universe except those parts occupied by terrestrial and planetary bodies, which are composed mostly of matter of the third kind. The constituent parts of this kind of matter, as stated before, consist of agglomerated masses of the other two kinds, whose pores are filled with matter of the first and second kinds still in their simple state. According to the relative proportions and various arrangements of these three constituents, we distinguish all the different bodies which are classed as solids, liquids, and gases. Of these, solid bodies are those whose least 
parts are made of closely packed and intricately arranged particles, which can move with respect to each other but very slightly, if at all. The spaces between the constituent parts of a liquid are greater and contain a larger proportion of the subtle elements than solids; while gases have their particles so separated that they approach fire in their freedom of motion. As he quaintly puts it : a body is a fluid when it is divided in many little parts which are free to move separately in various ways, and it is hard when all its parts are so interlaced that there is no action capable of separating them. Cohesion in hard bodies is caused by the lack of motion of their constituent parts, for no cement could be harder than rest, which is the quality the most contrary to motion. He does not tell us how to interlace and twist a number of spheres and irregular fragments together, so as to form compact and inextricable units of matter. To anyone but a great philosopher they would remain ropes of sand.

Descartes had spent much time investigating the experimental laws and phenomena of light and with notable success. One of his greatest ambitions was evidently to explain the nature of light and the mode of its transmission, for he discusses these in great detail in his Principia and refers to his solution of the problem constantly in his letters with much complacency.

As noted, the substance of the sun and stars is like fire in respect to its motions; for there is nothing 
more agitated than fire which can disintegrate even solid matter into its smallest parts, and then carry them away. So we should believe that the matter of the sun is celestial fire, very fluid and agitated, carrying off with it particles of the heavens. Although matter of the first kind is constantly rushing along the axis toward the center of the vortex, yet, when it approaches that point, it experiences a tendency to fly out to the equatorial surface because of its centrifugal force. The effort, not only of the little spheres of the second kind but also of the fragmentary particles of the first kind, to leave the center of a stellar vortex, constitutes light. While all particles of a vortex may tend to move away from the center because of their centrifugal force, yet, since they are packed like balls in a cup, they cannot really move and so only tend to move. Just as when a sufficient hole is made in the bottom of a cup filled with balls, one of them drops through and all the balls lying within an inverted cone settle down; so it is with light, if any particle in space tends to move, this tendency or pressure is instantly transmitted to any distance along straight lines, which come not only from the center of a luminous body but also from all points in its superficies. If we attempted to discuss details, we should find ourselves worried and baffled between this tendency to move in a plenum and the real motions of balls in a cup containing free spaces between them. But there is little use in fretting 
over such a detail, when the entire conception is not light nor anything resembling light. Descartes may, perhaps, prove that this tendency to move proceeds in straight lines and would be reflected and refracted in agreement with the laws of light; that the interstellar spaces would be transparent; that terrestrial bodies would vary from transparency to opacity according to the greater proportion and complexity of matter of the third kind in their composition; but the mechanism of all this is far more obscure and complicated than the phenomena it attempts to elucidate.

This theory of light advanced in the Principia was, in a sense, supported by the rather meager experimental knowledge then existing, and seems at first plausible. But examined critically, and with the mind no longer awed, or perhaps hypnotized, by Descartes's sweep of imagination and his power of making words seem to express clear ideas when they really do not, his cause of light is found to be as arbitrary as the fiat lux of a God, and its effects mere confusion. In fact, it is the unavoidable weakness of any such hypothesis, and a very irritating and tantalizing weakness, that the words used apparently express things we can understand, and yet when we try to visualize these things, stripped of technical and intricate verbiage, the mind has received no clear impression.

And here we have a splendid example of an hypothesis, whose foundations now seem ridiculous; whose 
laws are not correct generalizations; and whose conclusions are unlike the phenomena of nature; which, nevertheless, anticipates an idea to be advanced again, after a century and a half. This hypothesis of light contains, as a corollary, the germ of the kinetic theory of heat. He discards the notion, which then generally prevailed, that heat was a sort of mysterious substance called caloric, for which indeed there was no place in his universe, and defines it as the oscillatory agitation of terrestrial particles, set up by the pressure of light. The pores of such bodies are tortuous, and the pressure of light on one end of such a gross and irregularly shaped particle may be greater than on the other end and so cause a tipping motion. Such a tilt would give an oscillatory motion to a particle, similar to a violin string when bowed, and he believed its momentum affects us as heat. An oscillation of this sort would communicate itself to neighboring particles and therefore explains the conduction of heat from one part of a body to another; also, it accounts for the expansion of bodies by heat, since vibrating particles usually require more space than quiescent ones; but, on the other hand, they might be so shaped and arranged as to occupy less space, when in motion, and such a body should contract if heated. Such an effect was unknown at that time, but we ought to claim that it was brilliantly verified when, in later years, water was observed to contract when heated from zero to four 
degrees Centigrade. Not to contradict known phenomena, and to anticipate some unknown ones, is held to be the justification of such hypotheses; by this standard Descartes was successful as few others have been. Yet, if we compare the splendid advances which he made in science by his experimental discoveries and his application of mathematics to physical laws, to the mass of falsehood in his metaphysical schemes by which he dominated science and which still flourish in the metaphysical theories now in vogue, we can hardly tell whether he has benefited or done harm to science by his labors. Possibly no labor is too great, if by it we arrive at ever so little truth, but no one can believe that this medieval conception of light and heat induced his successors to seek for a possible, but unexpected, contraction of water. Surely the converse is the case and his metaphysical divination was purely specious; the phenomena were discovered without any reference to his theory and probably without even a knowledge of it, and now, if we wish, we may use them as a buttress for Descartes's tottering edifice.

The fourth and last book of the Principia is devoted to an explanation of the natural phenomena of the earth. At first the earth was a small stellar vortex, composed of matter of the first kind only, and was one of fourteen satellary nuclei located in our larger solar vortex. Gradually the less subtile parts of its mass attached themselves together and formed spots or 
crusts on its surface, similar to sun-spots, a phenomenon then recently made known by the telescope. Sun-spots, because of the superior agitation of solar matter, are broken up and disappear, but the earthspots formed more rapidly than they dissipated, finally covering its surface with a thick crust of many layers, composed of what he called matter of the third kind. This crust diminished the motion of the terrestrial vortex and finally destroyed it altogether, so that the earth with its atmosphere and its obscure bodies descended toward the sun to the place it now occupies.

With the earth in its proper position and with the beginnings of diversity in its composition, Descartes was free to employ the remainder of his treatise to the description of the character of each aggregation of elementary matter necessary to form the various chemical substances; the proportions and velocities of the three kinds of matter to produce forces, such as cohesion, weight, etc., and the different geo-physical phenomena, such as winds, rain, and earthquakes. It is amazing how much he passes before our eyes, and how ingeniously he links his ideas, giving to them the appearance of the greatest plausibility.

There is little profit in discussing these at length, for if the conclusions of the hypothesis were absurd when applied to those regions of space about which we even yet know little, they become grotesque when connected with matter which we can investigate experi- 
mentally. Besides our thesis is with such methods in general, and in this respect only is a study of Descartes's system useful, as it is his method which still flourishes in the theories of physics and of other sciences. $\mathrm{He}$ is to be admired in this respect; when he had once outlined his premises and his method, he deduced his conclusions as rigorously as possible, and compared them consistently with experience; whereas it has become the custom now to alter postulates whenever their conclusions point to error, with the result that it is most difficult to outline a consistent and invidual modern theory. If we study modern scientific theories we find that the postulates are as metaphysical, if not more so, than those of Descartes. They are stated with much assurance, but as the conclusions unfold themselves, we begin to notice a certain hesitation and a desire to limit the discussion to a small and related class of phenomena. Or if an excursion is made into a wider field, lack of confidence increases and usually results in a modification and confusion of the postulates. If Descartes's theory may be illustrated as a tree with all its conclusions branching out from a single idea as a stem, our present state of physics is like a thicket of bushes with many stems so concealed and interwoven that the parent stem of any branch cannot be distinguished.

I have sketched the most elaborate and comprehensive hypothesis ever developed in the name of science, 
which, if it means anything to mankind, is his best expression of verity and fact. Yet we see Descartes, an illustrious man of science, devoting his talents to the exposition of an openly confessed fiction. And his reputation rests on the belief that he has spun a web of fancy so subtly that it could deceive us. While additional knowledge has been acquired by us, no one has shown that modern theorists have discovered a method different and more trustworthy than that of Descartes. We recognize that many of the laws he formulated are false and that most of his facts have been corrected or disproved, but we should remember that modern hypotheses also are developed as a means of attacking unexplored regions of science where our own knowledge is either meager or false. For example, he felt it necessary to find a cause for the recently discovered sun-spots and then extended its action so as to change a vortex into a primitive terrestrial planet. A better knowledge of these spots on the sun proves that his whole reasoning was false, or shall I say childish. But was it less plausible at that time or even less childish than is now our most recent theory; that an atom of matter is a system of corpuscles, each of which is a unit of free negative electricity moving with the velocity of light, and that this denatured bit of electricity is nothing but a localized strain in an ethereal plenum. Let us examine such a postulate as we would a similar statement if it had been 
made by Descartes. We may admit with Lord Kelvin that we know nothing about the real nature of electricity. We do know experimentally that electricity seems to be associated always with matter; the greatest velocity we have caused or observed any body of an appreciable size to have, is one thousand eight hundred times less than the speed of light. An ethereal plenum is certainly only a matter of imagination, and the conception of matter as a strain in this imaginary plenum is hardly a clear idea. So it seems that the facts supporting our modern postulates regarding the nature of substance are as meager and doubtful, and our ideas as obscure for our purpose, as those of Descartes were for his.

On the contrary, it is no exaggeration to say that there probably never lived a man better equipped than Descartes to make and to defend an hypothesis; his scientific skepticism, his freedom from the trammels of authority, his devotion and skill in experimental work, his determination to submit his ideas to the rigorous logic of mathematical analysis in which he was the leader of his age, were admirable qualities for such a purpose. A man who doubts even his own existence, is not likely to adopt other ideas lightly. To strip matter of every quality except mere extension; to abolish every cause of phenomena except linear motion and the impact of bodies, both of which are capable of the most accurate experimental investiga- 
tion; is to attempt the limit of simplicity in scientific procedure. Why Descartes chose such a postulate as the criterion of matter, is directly traceable to his fundamental dictum: cogito, ergo sum. If his own existence is real only because of thought, and if mind or thought be the criterion of all reality, form replaces substance, and the extent of matter is its essential quality. Although Descartes went further in this direction than most men of science are willing to go, yet he has imposed his method on science to the present day and we are still industriously building worlds as we think them to be.

Dazzling as the system of Descartes appears when viewed as a whole, it has a foundation of sand and an imaginary rather than a substantial superstructure. Consider his statements: space or matter is infinite in extent and continuous in character, and in the beginning it was divided in equal parts which were then moved with a force sufficient to separate them. If matter fills all space, or rather is space, where was the additional space to permit of this separation and what then filled the interstices between the separated parts? Again, the impact and grinding of particle on particle are supposed to have reduced them to uniform spheres and the dust of this attrition then served to fill the spaces left in such a pile of spheres. This dust is of all shapes and so fine that there is always at hand just the proper quantity to fill any space be- 
tween the spheres. It really amounts to endowing these cosmic fragments with the intelligence of Leibnitzian monads; if impact and grinding produced them, why are they also not true spheres? The sand on the seashore is as spherical as the pebbles. Then too, if the original parts of space were reduced in size by attrition, why did they attain a certain size and then cease to be worn away? Or are we to suppose that the whole universe-earth, stars, and interstellar spaces -are still grinding themselves away until some day all its parts will be reduced to the size of the cosmic dust which he calls fire? We might go still further and let the universe actually grind itself into nothing, and simply vanish.

But even if we showed, step by step, that the scheme not only was not true, but even not capable of resisting the most cursory criticism, we should be met by the answer; that as knowledge increases, details which are erroneous will be abandoned and new ones substituted which better approximate to the truth. This countercriticism seems aside from the question; it would be strange if the efforts made to discover new phenomena and laws, and to correct false ones, did not increase our knowledge. But is this aim furthered by such hypothetical systems, which attempt to describe the mechanism of these phenomena and laws, and which, at bottom, assume that their authors are the creators of the universe and not observers of one whose laws and 
phenomena are independent of them; a confusion of subjective ideality and objective reality? Also however it advances, our knowledge of nature will always be so inadequate that the very announcement of a system of nature should cause us to suspect it of being etched out by fancy, and to be useless as an aid to scientific investigation. Nor can we find a system which does not transgress constantly the limitations of science, and it is safe to say none will ever be proposed which will not transgress them, because it is the desire for such a system that is false, and not its development.

On the other hand, the discovery and verification of phenomena should be unreservedly advocated, also their classification into laws and even the restricted use of hypothesis. But the latter has come to have two meanings in scientific usage. The word hypothesis very frequently signifies a law which has been pretty accurately expressed and verified by available experience, but which still does not embrace some phenomena believed to be related to it, or is contradicted by some others; for example, the law of conservation of energy was an hypothesis in this sense, until the discovery of the mechanical equivalent of heat proved that the mechanical energy, apparently lost in every action by friction, was accurately balanced by the thermal energy produced by the friction. Such hypothetical reasoning is quite warranted; in fact a law or hypothesis of 
this sort should always be announced as soon as a considerable number of facts point to its probable truth; such tentative laws always direct attention to the phenomena involved and stimulate research.

But hypothesis in the other sense does not gradually crystallize into law as our knowledge increases. No information, however greater than ours at present, will ever advance Descartes's hypothesis a step closer to a law. We shall never have any data about his three kinds of matter, his nature of free space, etc.; we learn constantly more about the action of light but we still drift confusedly and without a guide between his apparatus of pressures, Newton's corpuscles, and Huygens's waves; the latest treatise on optics now states that we may have to mix together all three of them.

The system of Descartes will fascinate anyone who surrenders himself to its spirit and scope, but illusion is not the function of science. Nor can I find any more accurate and just criticism of this and all other hypotheses than that given by Bolingbroke: "The notion Descartes entertained and propagated, that there is, besides clear ideas, a kind of inward sentiment of evidence, which may be a principle of knowledge, is, I suppose, dangerous in physical inquiries as well as in abstract reasoning. He who departs from the analytic method, to establish general propositions concerning the phenomena on assumptions, and who rea- 
sons from these assumptions, afterwards on inward sentiments of knowledge, as they are called, instead of clear and real ideas, lays aside, at once, the only sure guides to knowledge. This Descartes did very widely in his construction of a world, and yet by dint of genius he gave a great air of simplicity and plausibility to his hypothesis, and he knew how to make even geometry subservient to error. . . . The plenum of Descartes is well-nigh destroyed; many of his laws of motion are shown to be false; the mills that served to grind his three elements are demolished: and his fluid matter in which, as in a torrent, the planets were carried around the sun, whilst a similar motion in the particular vortex of every planet impelled all bodies to the center, is vanished. Notwithstanding all this, how slowly, how unwillingly have many philosophers departed from the Cartesian hypothesis?"

That Bolingbroke was mistaken when he says that the plenum, the vortices, and all the other apparatus of Descartes have been destroyed, can be readily seen by reading any modern treatise on physics.

But he was vividly correct in the larger and more important part, when he finds that all such hypotheses are based on an inward sentiment of truth and not on clear and real ideas. An inward sentiment of knowledge is and must be the final guide of anyone who employs this hypothetical method, for how can anyone have clear and real ideas about such things as tran- 


\section{I06 THE LIMITATIONS OF SCIENCE}

scend experience? And amongst the many following this method, what man can be the arbiter to declare which one has been gifted by a divine power with the true inward sentiment of knowledge? 


\section{CHAPTER IV}

\section{THE SCIENTIFIC METHOD}

The truth of science has ever had not merely the task of evolving herself from the dull and uniform mist of ignorance, but also that of repressing and dissolving the phantoms of the imagination.-FARADAY.

If we are compelled to revert to old and supposedly discarded systems of thought when we attempt to make new hypothetical systems, we should inquire whether we are really advancing the theory of science by that method. Is it true that Descartes reaches in his wonderful clarity of expression the highest attainment in speculative thought? Even in the then little cultivated subjects of electricity and magnetism, his imagination did not fail him and he drew a picture of the field of force about a magnet which is strikingly like those in modern treatises. And to explain electrical attraction, he supposed bodies to contain little filaments of his elementary matter which were crowded out when the bodies were rubbed together. These filaments attached themselves like lines of force to neighboring bodies. When the rubbing was stopped they retracted and so drew the electrified bodies together. If we modernize this explanation, we have a fair state- 
ment of Faraday's lines of force. Faraday believed that when a body is electrified the space about it is filled with lines of electric force which are stretched in the direction of their length and experience a pressure at right angles to their direction. This idea, or rather the modification of it by Maxwell, who was able to assign quantitative values to those forces which correspond with the laws of electrical attraction and repulsion, is expressed more precisely. But qualitatively: that is, in telling us what electricity is; why it is produced by friction; and what lines of force are; the modern statement is no more definite than that of Descartes. It is a mistake, however, to suppose he possessed a unique power of formulating hypothesis; other early writers attained eminence in this respect. Thus Sir Thomas Browne thought that electric effluvia (the prevailing name for force) behave like threads of syrup which elongate and contract and so produce attraction; Von Guericke stated that bodies contain effluvia which emanate from them according to their nature and form an electric field of force. In agreement with these opinions, we are taught to-day that the best way to consider lines of force is to picture an electrified body as one surrounded by stretched elastic bands. As a diagram to show the direction of motion of an attracted body, and as a name for the quantity of force, this conception of elastic lines of force is accurate and convenient. But Faraday and Maxwell went far be- 
yond this, as they gave to them a concrete reality. This conception still persists, although Sir J. J. Thomson showed years ago that no known system of mechanical forces would keep such a system of force-lines in equilibrium and Professor Lorentz now says they cannot really exist but are fictions of the imagination.

It seems rather futile, if such be the normal history of hypothetical models, to inflict on us the labor of learning abstruse hypotheses which continually revamp old metaphysical terms and merely dress them up in new transcendental symbols. It is a valuable exercise to strip hypotheses of their technical phraseology; to change those words which deceive our minds into believing that a clear idea has been conveyed, when, in fact, they have merely been wrenched from any real significance. Thus Sir Oliver Lodge says that the ether is very massive. This definition at once increases our belief in its reality since it conveys the impression that the ether is tangible and impenetrable, something like a vast globe of rock. And we must stop to think before we realize that whatever the ether may be, it must be just the opposite to our ordinary ideas of massive things. Or what clear idea is conveyed by Professor Einstein's definition that vacuous space contains radiant energy which is an entity of the same kind as matter? Does he not add to the difficulty when he says further, that the difference between a vacuum and the ether is that the latter is a vacuum transmitting radiant 
energy and possesses a light vector. What right has he to insinuate into our minds that a vacuum may contain something and still be a vacuum? He does this by a play on the word "energy," which he permits us to think of in the ordinary sense as an attribute of matter and at the same time states implicitly to be a distinct entity. We are inclined to pass lightly over the second statement because we tacitly think of a light vector as a geometrical line; the substance of such a line is too intangible to give a distinct shock to the reason. But if we put this definition to a simple test, we easily see how futile it is. Say to anyone, that a golf ball in its flight is not a thing of rubber and paint but a complex of energy; and, that this is true because the moving golf ball has a motion vector and consequently changes vacuous space into ether. How quickly such a statement about a familiar action would be recognized as an absurdity. I presume that the reason why we like to indulge in these phantoms of the imagination is because we still hate to confess our ignorance. But it is a pity that science thus lurks in the mists.

We can easily follow modern hypothesis as it progresses into transcendental symbolism. Sir Joseph Larmor and Professor Lorentz wish to define electricity so that it may be measured. They adopt the hypothetical method to a limited degree. Thus, they each postulate the atom of matter. They next assume each atom to consist of a multitude of positively and 
negatively electrified particles which revolve in orbits. Except to say that these particles may be a modification of an ether, they avoid explicit details. Their method of exposition is still the classic method of mechanics. When they try to explain certain lately discovered and obscure phenomena, they merely show that they could be satisfied by supposing the dimensions of bodies to be influenced by their motion. At this point they wisely refrain from further explanations. Next we find that Professor Einstein definitely changes electrical particles into particles of electricity and matter into complexes of energy. He speaks so confidently of his ability to visualize electricity and energy that we feel our own inability to do so results from a pitiful lack of intelligence. At this point he clinches the whole matter and explains the changes of the dimensions of moving bodies by introducing the occult idea that light is an entity which moves in space with a constant velocity. It is a far cry from the inductive method of science which attempts to build generalizations on experience, thus to make the whole concrete world conform to so abstract an idea as the constancy of the velocity of light in space. Nor does he hesitate to found physical science on the paradox, that motion cannot be absolute but the motion of light is absolute. Then Professor Minkowski goes a step further. He accepts all these ideas and then treats them symbolically. To deduce conclusions from these postulates, 
he uses mathematical equations as if there were four dimensions to space. The mathematician can employ equations which contain four or any number of variables, but the physicist who desires to deal with an objectively real universe and also to be intelligible is forced to limit himself to the three dimensions which correspond to his powers of measurement of length, breadth, and depth. Lastly, Professor Lewis confuses scientific method utterly by arbitrarily assuming which quantities in an equation shall be treated as variable and which as constant. Thus he says, if the momentum of a body changes, let us suppose that this happens not because its motion changes but because we shall consider its mass variable. Of course anyone can say, let us consider the universe to act as he wishes. But, after all, what is the use when no one believes it does? Is it any wonder that a gulf is growing not only between men of science and the rest of the world, but also between theorists and those who are still willing to submit their imagination to experience? Such a gulf is certain to continue so long as theorists are willing, and even anxious, to ignore common sense and the facts gained by patient and exact experimentation.

It is true that my criticism of scientific method has been so far purely destructive. And in that admission may lie the ineffectiveness of the argument to many. Some will say that however weak and vacillating the 
hypothetical method may have been, yet it is the custom of thought and in spite of it or even, in some way, because of it, scientific truth has nobly expanded, dispelling ignorance and subjugating natural forces. But because knowledge advances, objection should not be made to a protest against what tends to embarrass a more rapid advance. Such a protest is the value of destructive criticism. The reason why scientific knowledge advances in spite of hypotheses, those phantoms of the imagination, is because hypothesis rarely precedes experiment. If we examine the work of the experimenter of to-day, we find he still goes on calmly working with apparatus and using it with the common sense idea that he is using real objective matter in spite of the attempt of the theorist to make it an attribute of energy or electricity. The conclusions derived from experiment and laws may be discussed and condemned or approved according as they support a fashionable hypothesis, but scientific hypothesis is much like religious dogma: although it may protest, yet in the end it swerves around to accept all new facts. And in a short time the despised fact is cited as a pillar, or at least as a flying buttress of the hypothesis.

While it is not possible to draw a definite boundary line between the regions of physics and metaphysics, still we may do so in a general way by saying that the domain of physics is concerned with the discovery of phenomena and the formulation of natural laws based 
on postulates which are determined by experience and generally accepted as true; the causes of phenomena and the discussion of the postulates of science lie in the province of the metaphysician. This differentiation in method of thought cannot be rigidly adhered to since the boundary line is more or less obscure, and is liable to considerable displacement as a science advances; but the acceptance of this principle would prevent much of the confusion which has been introduced into science by writers who have not recognized it to be a general rule. For example, the principle of relativity is not strictly a physical law but the expression, in mathematical symbols, of the general philosophical law of the finite nature of the human mind which has been accepted for centuries. Again, the discussion of the shape of the atom or electron is not a physical problem, as it is incapable of verification by experience. This does not mean that such questions should not be discussed, but the method of their discussion and the results obtained are properly the method and results of metaphysics and are not in the category of physical phenomena and laws.

In the first place, men of science must accept the existence of an objective universe, whose phenomena and laws are external to our intelligence and will. But, since the interpretation of phenomena is subjective and thus conditioned by our intelligence and will, scientific laws are governed by the mathematical 
theory of probability, or the consensus of opinion of the greatest number, working with the greatest care.

The basic laws of physics are the laws of continuity and conservation, and the law of cause and effect. Unless we believe that something, matter, energy, or both, remains unchanged in amount and has a continuous existence however changed in appearance, and unless phenomena can be repeated, we have no certainty of knowledge and no means of communicating ideas to others. The discussion of the reality of these laws may be left to the philosopher, but I dare say, however he decides, they will continue to be accepted by mankind generally.

The universality and application of these and other laws should be rigidly limited to experience by the physicist. The law of conservation requires that the quantity of matter and energy is either infinite, which means nothing, or that there is an exact interchange in quantity in every action. This belief is expressed in Newton's law that to every action there is an equal and contrary reaction. But it is evident that conservation, as a scientific law which may be verified, is limited to a very small category of observations and is only approximate for them. Thus we know nothing of the total amount of energy radiated from the sun or where it goes. Hypothesis here shows considerable vacillation. Some assert that the universe is infinite and so the radiation never reaches a boundary; others say 
that the universe is so filled with matter that no straight line can be drawn from the sun without reaching an obstacle and so radiant energy is reflected back and forth; again the universe may be finite in size and its boundary may be a reflecting surface. These hypotheses are evidently futile speculations and no support to the law which we have been driven to accept and shall continue to accept until personal observation shows results which increasingly depart from the law.

So, too, the law of cause and effect is a generalization from few observations and neither supports nor is supported by hypothesis. While we can never hope to establish such a law, it is nevertheless a necessary generalization, or scientific deduction becomes meaningless. The fact is, the phenomena of the universe do not reveal themselves, as a whole, in any regular sequence of cause and effect; and our theories, based on such a law, show such a complex tangle as to be quite beyond our power to interpret. The law involves time, and past time at that; and the successive causes of an observed phenomenon, if carried back in any logical sequence, soon widen out into an incomprehensible maze and vanish in the obscurity of the past. The most beautiful and perfect example of this law is the belief in organic evolution. Yet on what meager and inaccurate observations it rests. Everyone believes in some such law, but no one can point out the sequence of cause and effect, and its rigorous development leads 
to absurdity. Mathematically it is a law of geometrically decreasing types which reduce finally to a single protoplasm. If we adopt the hypothetical method, we should discuss the origin of this protoplasm. To say it was made by God, is not scientific. Lord Kelvin offers an hypothesis (although one would suppose he was joking if the context were not so serious) when he suggests that the original protoplasm may have fallen on the earth from some celestial body! However we are to consider such an extraordinary statement, we do know that Maxwell tried to support the hypothesis of pangenetic evolution by the futile method of estimating the number of molecules in a germ. The question with him was whether there were enough molecules to form miniature organs and parts of the mature object into which the germ would expand.

Another illustration may be given to show that as soon as we extend our laws beyond the point where verification is possible, we merely cause confusion. Certain experiments indicate that the apparent mass of a body changes when it moves in an electric field. The law shows that the mass should become infinite when the velocity equals that of light. This conclusion has been accepted as the basis of an hypothesis that the velocity of light is a maximum limit to motion. Such a belief is not only pure hypothesis but it is contrary to the conclusions of other hypotheses. If this hypo- 
thetical method is generally applicable it is necessary to explain gravitational action also as due to some form of motion; Laplace has shown that the motion involved requires a velocity many times that of light.

Our most fundamental perception of an external universe seems attainable from our sense perception of force. The muscular sense of effort is apparently the simplest and most general mechanical notion we have, and in the opinion of the masters of the science our idea of force is adequate to serve as the basis of so exact a science as physics. Yet, when we attempt to make force serve as a fundamental measure of phenomena, we have found, since the time of Newton, that it is not so convenient as mass and acceleration. Motion is further divisible into a measurement of length and time. It is therefore customary to reduce all our complex observations into combinations of the fundamental units of mass, length, and time instead of force. The idea of force being thus associated in our minds as an attribute of matter, we postulate the objective reality and conservation of matter and assume the fundamental attribute of matter to be its mass or inertia. M. Hannequin expresses this idea clearly when he says that nothing in a mechanical sense exists except masses in motion.

We shall next assume length, mass, and time to be the fundamental units of measure. These quantities are continuous or, at least, are indefinitely divisible. 
The continuity of space and time is generally accepted; without this belief it is impossible to establish the geometrical laws of figure founded on the point, line, and surface or the analytical laws of motion derived from the calculus. The only exception I know to this postulate is Professor Planck's theory of quanta, in which motion may occur in jumps. But the divisibility of matter is not usually supposed to be infinite. Indeed, the denial of this assertion is the foundation of all atomic theories. Yet it is difficult to see how mathematics can be anything but abstract logic, or how it can be applied to physical problems unless this third fundamental quantity, which is, as it were, the connecting link between the abstract and the concrete, be also indefinitely divisible. It is only by the postulate of the indefinite divisibility of mass that we may pass from the mathematical laws of pure motion (kinematics) to the physical laws of the motion of bodies (dynamics). How, otherwise, can we replace finite bodies by mathematical centers of inertia? In this connection Sir Joseph Larmor says: "The difficulty of imagining a definite uniform limit of divisibility of matter will always be a philosophical obstacle to an atomic theory, so long as atoms are regarded as discrete particles moving in empty space. But as soon as we take the next step in physical development, that of ceasing to regard space as mere empty geometrical continuity, the atomic constitution of matter (each 
ultimate atom consisting of parts which are incapable of separate existence, as Lucretius held) is raised to a natural and necessary consequence of the new standpoint." This is clearly an attempt to reconcile the two antinomies of continuity and discontinuity, which are usually attached to the names of Descartes and Lucretius. This Sir J. Larmor tries to do by postulating the existence of a true matter, which is a continuous plenum and imperceptible to our senses, and relegating sensible matter to the rôle of a mere variation in this otherwise changeless plenum-making it an attribute rather than an entity. If this definition denies the infinite divisibility of matter, it apparently accepts its indefinite divisibilty; the atom, as a variation limited only by our power of observation, must become smaller with each advance in the refinement of our apparatus. Such a plenum must remain a pure creation of the imagination, and its existence is not determinable by physical or experimental methods; it must therefore be classed as a problem for the metaphysician. The distinction between atoms continually diminishing in size and the infinite, or at least indefinite, divisibility of matter is here a question of words-the definition of what matter is.

Few things have been brought out more clearly by modern physicists than that, if we accept the doctrine of the continuity and conservation of energy, either of the two, matter or energy, may be considered as the 
fundamental unit from which the other can be derived. This undoubtedly follows from the fact that we have no conception of matter without energy or of energy without matter. But while it is thus possible mathematically to make either of them a starting-point for the explanation of phenomena, the advocates of energetics apparently soon develop a pronounced tendency to prefer the abstract to the concrete and to subtilize objective facts into metaphysical ideas. A science like physics, to be useful and not merely an intellectual gymnastics, should preserve in all its speculations a close touch with the practical and the concrete-a certain common sense. The history of the science shows these advantages have been obtained most frequently by those who maintain matter and not energy to be a fundamental entity. The failure of the mechanistic school has arisen from the attempt to explain the nature of matter, the cause of its forces, and the properties of atoms. However we may try to reason away the belief in the objective reality of matter, our minds persistently cling to the advantage, and even necessity, of such a postulate, and we consciously or unconsciously endow any substitute of it with all the properties of matter, excepting its name.

Energy is to be considered then as an attribute of matter and may be defined as the power to do work. It is customary and convenient to divide energy into two classes, potential and kinetic, according as the mat- 
ter concerned appears to be at rest or in motion. Since attractive forces exist between all bodies, we find that the idea of work is involved whenever the distance between bodies is altered and we denote this potential energy as the force of attraction times the distance between them. I accept frankly the idea of action at a distance, not because it seems reasonable, for my experience teaches me that to cause motion I must construct a link between two bodies; but the fact remains, that both gravitational and electrical attractions seem to act at a distance and all attempts made to explain them have so confused the mind that the occult phenomenon is less perplexing than the occult explanation. In the majority of such problems we can go no further; but in certain cases, as for instance the theory of gases, a portion of the potential energy due to the pressure of gases may be ascribed to the impact of small moving masses of gases. Even here, a portion of the energy of the gas must be considered as potential and incapable of further explanation. Kinetic energy is the power of work due to the motion of bodies. Its measure is one half the moving mass into the square of its velocity. These two kinds account for all the energy of material bodies; they are mutually interchangeable and their sum is a constant.

A third class of energy remains to be discussed. It is called radiant energy; its difference lies in the fact that it is not associated with material bodies. Cer- 
tain forms of the energy of bodies which we name heat, light, etc., are observed to appear in other bodies although the space between them is devoid of matter. We suspect that the amount of this energy disappearing in the emitting bodies is equal to the amount appearing in the receiving bodies and that time elapses between the emission and the reception of the energy. On these ideas we have built the theory that energy travels through immaterial space and in its transit we call it radiant energy. The conception of radiant energy in a vacuum is a useful one if we limit it to a mere quantitative idea. For this purpose it suffices to state the facts in a mathematical formula which expresses a continuous passage of a quantity of energy through space equal to the amount lost by the emitting body. We thereby generalize the laws of continuity and conservation and of cause and effect. This is not the custom; it is usual to try to explain the nature and mechanism of radiant energy. This requires either that space be filled with an ether to serve as the vehicle of energy or that energy be regarded as itself an entity.

It has been the persistent attempt of physicists for centuries to explain this radiant energy by mechanical models. And this effort has fastened on the science an interminable series of impossible fictitious ethers and mechanical atoms. The most indefatigable labors of the greatest minds have been spent to construct an 
atom, which would serve satisfactorily as a source and, at the same time, as a receptacle of radiant energy and an ether which would transfer it. Not one of these models has been even partially adequate; the course of the development has been steadily from the simple to the complex, from the concrete to the abstract, from the physical to the metaphysical, until the most recent atom is a complex more intricate than a stellar cosmogony, whose parts are an entity called electricity, and the ether is an abstraction devoid of any mechanical attributes. Out of all this controversy we have gained the following facts:- Heat, light, and electrical energy, originating in one body, may be assumed to pass through space undiminished and unaugmented to another body. We can also express this energy as kinetic energy while it is associated with matter. In transit, since our experience gives us no clew or criterion, we can assume as a formula for the energy, either a periodic motion of an hypothetical something, called an ether, or a projectile motion of an hypothetical massparticle. In either case, all we really do is to divide the initial or final material energy into two mathematical quantities, one a mass-factor and the other a velocity-factor, and give to each such a value as to make their product remain a constant. As a rule, we make the mass-factor so small that we can shut our eyes to its existence and imagine anything about it we please. The time relation is fixed by experiment. 
For the purposes of theory, although this energy appeals to our senses in the three forms of heat, light, and electricity, which in their qualitative aspects are each fundamental and not referable one to another, we fortunately find that quantitatively all three are satisfied by one dynamic formula. We have therefore obtained an adequate quantitative knowledge of energy, but not an inkling of the qualitative coefficients in this formula.

The hypothesis of the ether is an attempt to accomplish the impossible. And while it is now generally admitted that we cannot create such a substance as will satisfy the physical requirements of a transmitter of radiant energy, still the ether is claimed to be a useful hypothesis. This utility is said to consist in giving us a crude image, in a mechanical way, of what occurs. In other words, it supports our innate dislike to confessing complete ignorance, and it provides a set of concrete analogies for abstract statements and equations. Now the old elastic solid and mechanical ether did afford us a concrete image of a mechanism for radiant energy, and we could speak of it with some intelligence to one another, because everyone has a conception of an elastic solid. To be sure, this solid ether became a grotesque. It permitted the transference of heat and light energy, but only at the expense of creating a kind of matter entirely outside of, and contradictory to, anything in our experience. We have only to recall the 
properties ascribed to this ether to find that it operated equally well if it had a density indefinitely great or one indefinitely small; if it were rigid or if it were collapsible, etc. As certainly as one physicist endowed it with a property, another arose who showed that just the opposite property was equally efficient. Yet we might still be staggering along with the conviction that somehow this supposititious stuff was of use to us; at least it gave us a set of words conveying some meaning. But when Maxwell proved mathematically that a third kind of radiant energy of an electrical type should be looked for, and when Hertz demonstrated its existence, no elastic solid would serve for all three kinds; and so, for a time, we were taught simultaneously the properties of two coexistent ethers. An elastic solid and a so-called electro-magnetic ether in a single space were impossible, and the former soon collapsed since it was more concrete and thus more vulnerable to criticism. Maxwell's idea produced a revolution in the theory of physics; heat and light remained no longer a form of mechanical waves but became electro-magnetic waves of a special periodicity. By a progressive subtilization we have now arrived at Sir J. Larmor's celebrated definition of a medium which will satisfy all forms of radiant energy. The ether is " $a$ plenum with uniform properties throughout all extension, but permeated by intrinsic singular points, each of which determines and, so to speak, locks up permanently a 
surrounding steady state of strain or other disturbance." This ether is unaffected by any type of mechanical action since ethereal strains are of an unknown kind responding only to electro-magnetic stresses. This definition seems general enough to satisfy the most critical, but Professor Einstein goes much further. He says we must abolish the ether because the only difference between empty and occupied space is that the latter is the seat of an entity, energy, and contains a light vector. Such a definition, in the sense of explaining a complex idea in terms of simpler ones, is wholly incomprehensible and at the same time apparently denies and affirms the existence of the ether. But Professor Einstein is not averse to paradoxes. These ideas evidently reduce matter to an attribute of electricity, and make all forces of the type called electrical forces. But if electricity is everything, we must inevitably some time explain pure mechanical actions in terms of this electrical substance. Sir J. Larmor clearly foresees this, as shown by his statement: "The electric character of the forces of chemical affinity was an accepted part of the chemical views of Davy, Berzelius, and Faraday; and more recent discussions, while clearing away crude conceptions, have invariably tended to the strengthening of that hypothesis. The mode in which the ordinary forces of cohesion could be included in such a view is still quite undeveloped." He thus rather leaves this question in the air by con- 
cluding that a complete theory is not necessary. But the history of science shows that we shall soon create two media or try to give properties to one which will include electrical, chemical, and material forces; indeed, this latter is already being attempted. If the conception of an elastic solid ether was admittedly a fiction of the mind, and one impossible to align with any known kind of matter, the electro-magnetic ether is so esoteric, so subtilized from all substance, that it merely provides a nomenclature for a set of equations expressing the propagation of radiant energy. We may well go still further, for I believe the time is rapidly approaching when all scientific discussion of the nature of the ether will be considered futile.

In accordance with my view no hypothesis will be made to express properties of a medium, whose existence is itself incapable of scientific proof. It is, at the same time, perfectly proper to distinguish space through which we say radiant energy passes by a special name such as the ether. The amount of radiant energy in transit is best given by an equation expressing conservation of energy and containing a velocity and an inertia factor. The velocity factor of this equation most conveniently takes the form of a periodic motion, but no assumptions need to be made as to the nature of the periodicity or of the inertia factor, since they also are not subject to experimental verification. 
Such a revolution as has occurred in the ideas of the ether requires a like one in our ideas of matter. The most notable effort in theoretical physics, at the present time, is the hypothesis that the ultimate element of matter is not a material atom, a sort of microcosm of sensible matter, but a free electrical charge, considered to be an entity for the purpose; added to this are the dependent ideas that inertia and all other properties of matter are attributes of electricity. This hypothesis can mean nothing else than that the Lucretian atom, the centers of force of Boscovich, the vortices of Kelvin, and all the atomic models (made of weights and springs and strings), have failed and become useless as aids to the imagination.

Sir J. Larmor defines this new atom as a protion, " in whole or in part a nucleus of intrinsic strain in the ether, a place at which the continuity of the medium has been broken and cemented together again (to use a crude but effective image) without accurately fitting the parts, so that there is a residual strain all round the place." This strain is not of the character of mechanical elasticity, since the "ultimate element of material constitution is taken to be an electric charge or nucleus of permanent ethereal strain instead of a vortex ring." Sir J. J. Thomson pictures the atoms of the various chemical elements as nuclei of free positive electricity holding in electrical equilibrium free negative charges, placed in various geometrical designs. 


\section{THE LIMITATIONS OF SCIENCE}

The degree of stability of each system is determined by the radio-activity of its element. Professor Lorentz considers the protion to be a small particle charged with electricity and probably a local modification of the ether; but his work on electro-magnetic mass leads one to the opinion that he believes electricity to be the real essence of the material universe. The modern school of German physicists is frankly endeavoring to give a purely electro-magnetic foundation to the mechanism of the electron and to mechanical actions in general.

Now to me, and I believe to many men of science, the chief and indeed only value of an atomic theory is to give a concrete, though crude, image of matter reduced to its simplest conditions. The word electricity gives me no such image of matter; it conveys absolutely no idea of materiality nor even of space or time relations. What the originators of the electrical atom have done is apparently to transpose the words, matter and electricity, tacitly giving to the latter all the ideas usually associated with the former. We may as well take the next step at once and raise the objective universe on the Leibnitzian monad or on Schopenhauer's philosophy of "Die Welt als Wille und Vorstellung."

Again, the law of the conservation of matter has been one of the most fertile ideas in science; according to this law at least one attribute, inertia, remains constant however all others may change, thus giving con- 
tinuity to material bodies as well as to space and time. It is quite possible to imagine an element of this new electric matter to be composed of equal quantities of positive and negative electrons, whose motions are so balanced as to make all material attributes vanish and produce a quasi-annihilation of matter.

Lastly, when the statement is made that the electron is merely a local modification of the all-pervading ether, some idea should be given us as to the nature of this modification. If it is of the character of a strain, no meaning is conveyed unless this strain is subject to the laws of static or kinetic mechanics. But we have no knowledge of a static strain which fulfils the requirements of matter, especially that it must be localized at definite points and must be uncreatable and indestructible; of kinetic strains, the only one at present available is the vortex ring of Helmholtz and Kelvin. To imply that matter is electricity and that electricity is a static strain or a vortex ring, is to make an impossible assumption and is reasoning in a circle. If the vortex ring of matter failed chiefly because Maxwell said: "That at best it was a mode of motion and not matter as we know it," what chance has this new type to survive criticism?

Although matter appears to us as a continuous quantity or at least as divisible far below our present methods of experimentation, still it is convenient to give to the smallest observable portion of matter some 
such name as protion. This unit of matter must be reduced in size as refinement of observation increases, so that we may always be able to discuss it mathematically in the aggregate only. It must also be endowed with the same attributes which we recognize in gross matter.

At the present time this protion is the electron, and the only attributes necessary to assign to it are inertia in the Newtonian sense, a force of gravitational attraction and a force of electrical attraction, either positive or negative in sign. No causes for these attributes can be given, as they are fundamental. If the experiments of Kaufmann, which show that an electrified particle in motion has an apparently increased momentum, are cited as supporting the view that inertia is a function of velocity and should be considered as an attribute of an invariable quantity, the electrical charge, I hope to show that it is possible to accept Kaufmann's results and at the same time the invariability of inertia. Before proceeding further with this discussion it is convenient to assemble the foregoing ideas in a more concise form.

We have first postulated a real and objective universe and assigned to matter rather than to energy the rôle of being an entity. The fundamental attribute of matter which makes it recognizable by our senses is force.

We next assumed that quantitatively all phenomena 
may be expressed in mechanical ideas and that the fundamental units of measurement are mass, length, and time. These are continuous functions and consequently indefinitely divisible.

While matter, as measured by mass and extent, is indefinitely divisible, it is convenient to adopt as a scientific unit of mass a quantity so small that it is inferior to our powers of observation and so must be treated mathematically only in aggregates. As this is a general definition, the name protion has been given to this unit in order to avoid confusion with the chemical atom and the electron. At the present time the protion is the electron.

The general laws governing actions are the laws of continuity and conservation and the law of cause and effect. These are generalizations from experience and cannot be extended beyond possible experience without great precaution.

Energy is defined as the power of doing work and is held to be an attribute of matter. True or observational energy is always associated with matter and is divided, for convenience, into potential and kinetic energy.

A third and hypothetical kind of energy is assumed in order to extend quantitatively the laws of continuity and conservation, and of cause and effect, to the apparently well-founded interchange of energy of two bodies situated in a vacuum. This energy is named 
radiant energy and, in order to link it with kinetic energy, its quantity is expressed as the product of a "mass" and a "velocity" factor. Its velocity is naturally taken to be the distance between the bodies divided by the observed time. It is most conveniently expressed as a periodic motion with a translational velocity equal to $3 \times 10^{10}$ centimeters per second. No hypothesis is made in regard to the nature of the mass factor; it is taken merely as a coefficient to maintain conservation.

As no attempt is made to account for the mechanism of radiation, no attributes need be assigned to an ether. In fact no ether need be postulated, although it is advisable to use the name to differentiate space when it is occupied by radiant energy.

Action at a distance is a matter of experience and cannot be denied until some reasonable proof is found to account for force by some other means.

So far these ideas have had the approval of some acute men of science, however they may be disapproved of by others. Those which follow are more novel and need to be supported.

Since mechanical explanations are to be avoided, it is necessary to endow the electron with all the attributes of gross matter. For example, the protion of oxygen has a different density, force of cohesion, etc., from that of hydrogen. In this discussion, it is necessary to discuss only a few of these attributes. 
I shall, therefore, assume that the electron has a constant mass, $m$, and that it possesses a force of gravitational attraction for all other electrons which is determined only by their masses and the distance between them.

The electron, in addition to gravitational attraction, has a power of electrical attraction. To measure this force, I shall assume that it possesses a quantity of electricity, $e$. Electrical force obeys the same law as gravitational force, since it is conditioned by the quantity of electricity and the distance between electrons.

The electrical property of matter is also manifested to us by the experimental fact, that an electrified body apparently possesses a greater mass when in motion than an unelectrified body. A similar effect is noticed when a body is moved in a fluid. As the apparent increase in mass is there due to the resistance of the medium, it is called hydrodynamic mass, we may call the apparent increase in mass of a moving electron, its electro-magnetic mass $m_{e}$. The total or effective mass is therefore $m+m_{e}$.

Since the total mass is found to vary with the velocity of an electron, two suppositions are possible. Most physicists now assume that the mass, $m$, is a variable and that the mass, $m_{e}$, is constant. It seems to me more rational and more convenient to adopt the converse idea that the electro-magnetic mass, like the hydrodynamic mass, is a variable. I shall, therefore, 


\section{THE LIMITATIONS OF SCIENCE}

assume that the electrical charge on matter is a quantity varying with the velocity of a body.

So great a revolution in thought as to consider inertia a variable quantity and to substitute electricity for matter as the substance of the universe, would only have been undertaken from a fancied necessity. A mere matter of convenience would scarcely warrant the labor of revising the work of the past and of discarding what has been considered, until lately, as definitely established. The need for some such radical change in theory is based on the experimental facts discovered in connection with the passage of electricity through highly rarefied gases, and with radio-activity.

We may consider it established that the phenomena noted, when electricity is discharged in a high vacuum, are most readily explained by supposing the current due to a stream of electrified particles moving with a velocity comparable to light. The experiments of Sir J. J. Thomson and C. T. R. Wilson go to show that the masses of these projectiles, when charged negatively, are about the one-thousandth part of the mass of a hydrogen atom, provided the charge on each is assumed to be the same and equal to that of the hydrogen atom. Those charged positively are comparable to the various chemical atoms.

Radio-activity, on the whole, is best explained by the projection of positive and negative electrons from a certain class of bodies. 
And lastly, Kaufmann has shown by a delicate experiment that the apparent mass of an electron is a function of its velocity. This conclusion has been confirmed by others, although in minor points there is a considerable difference in results and opinions.

It must not be lost sight of that all these experiments deal with quantities of matter, supposing it to exist, too small to be appreciable by either chemical analysis or mechanical apparatus, such as the balance. They are ultimately measured by the force of electrical attraction of an electrical charge. We are, therefore, experimenting with matter which appeals to us through only one of its attributes. Is it not almost inevitable that an exclusive attention paid to this single attribute is likely to exalt it into an undue prominence? We have had, in the past, examples of much the same sort of reasoning. When the phenomena of light were predominantly discussed, physicists drifted into the opinion that this property of matter could be explained only by creating a light substance. Again, this process of reasoning occurred when heat was first investigated; we had the creation of caloric. And now we are asked to do the same thing with electricity. It is safe to predict that history will be repeated again, and that electrical charges and their forces will also sink into the condition of an attribute of matter.

It might certainly be true that two experiments showing equal electrical charges would, if we could 
measure the amount concerned, provide us with unequal quantities of matter, just as conversely equal quantities of matter might show different quantities of electricity. The hypothesis of equivalence of electrical charge and matter rests solely on an analogy to electrolysis, where matter is in a quite different state and also where the equivalence may be only approximate. Matter, on the other hand, in a solid state shows no connection between volume and density and electrical charge. In dealing with electricity we should not forget the immense superiority of electrical detectors in delicacy to those for mechanical quantities, so that we can appreciate far smaller quantities of electrified than of neutral bodies.

There is no doubt, from the quotations given, that theorists are basing their work on the assumption of the electron as the unit of matter. And they give to it the following properties: Its mass is wholly electromagnetic; the motive forces are electric forces; and the laws of mechanics are to be deduced from the laws of electro-magnetism.

At first sight, it would seem to be a simple matter to devise an experiment which would decide whether the mass or the electrical charge of matter is constant. But so far these two quantities have not only been found to be inseparable but they invariably enter as a simple ratio, whose value decreases with increasing velocity. Such a relation can, of course, be satisfied 
by assigning a proper variation either to the numerator or the denominator. It therefore becomes a mere matter of expedience which of the two quantities, mass or electrical change, shall be supposed constant. In addition, the quantity, $e / m$, is itself a constant for all velocities which can be attained by bodies which are appreciably large. So the whole question of variation is more or less academic, in that it does not become important unless we are discussing hypothetical atomic systems.

To say that $e$ is a constant is an assumption based solely on an analogy to the experimental laws of electrolysis; but in electrolysis, when we obtain equal electrical charges we also find equivalent masses of matter. In the discharge of electricity through gases and in radio-activity the matter deposited is too small to be measured. This is a fundamental difference and vitiates an analogy between the two. For example, we measure the amount of current in a vacuum tube by an electrical device, and at the same time we measure the deflection of the current by an electric and magnetic field; in other words, all quantities and forces are electrical, and we say that equal currents in this case require equivalent quantities of matter. But it has not been shown to be impossible or even improbable that electrons, associated with equal quantities of matter but having different velocities, might show different electrical charges; or that electrons producing equal 
electrical charges, might deposit different amounts of matter if it were sufficient in quantity to be detected by chemical or mechanical reactions.

As an hypothesis, I propose that, in order to make the ratio $\frac{e}{m}$ agree with the experimental evidence of its value and to account for electro-magnetic mass, we consider $m$ to be the mass of a particle of matter in the Newtonian sense, of constant and small value, and $e$, the electrical charge, to be a force attribute of matter which varies with the velocity of the particle.

However novel this hypothesis may be, I have not been able to find any experimental facts more difficult to explain by it than by any of the other hypotheses which have been recently advanced; and, on the other hand, it apparently accounts for much of the modern work in terms of old and well-established ideas.

From the very nature of my conception of the limits which should be imposed on scientific inquiry, I make no attempt to explain the cause for this electrical property of matter any more than I should for its gravitational attributes. Both are fundamental phenomena to be accepted as experimental facts until we gain contrary knowledge. Indeed, I have ventured to indulge in this speculation rather with the idea of showing that the recent hypotheses for electricity and matter; for the ether, protions, and corpuscular light; for the electro-magnetic and other non-Newtonian mechanics, are not necessary. We may still account as adequately 
for all our experimental facts by a simple addition to the attributes of matter and continue to base our theories on mechanical laws.

So long as the measurement of physical qualities becomes ultimately a matter of measuring mechanical forces, it is advisable to express quantitative physical laws in terms of mechanical formulæ. For this reason electricity should be considered a function of mechanical energy rather than the converse. If it be possible to place mechanics on an electro-dynamic basis, it is certain that we may always explain electricity in terms of pondero-dynamic laws. As both are possible, it seems far more natural and more rational to consider electricity as an attribute of matter than matter as a phenomenon of electricity. 


\section{CHAPTER V}

\section{THE CLASSICAL AND THE NEW MECHANICS}

Mathematica, quae philosophiam naturalem terminare, non generare aut procreare.-BACON.

So far the hypothetical method has been discussed in its broader aspects. It is my purpose now to outline its effect on science and on men of science by considering certain special cases. I know of no better way to point out the influence of this method on science than to contrast the classical mechanics, founded on the work of Galileo, Descartes, and Newton, and developed in accordance with the abstractive or realistic method, with the new mechanics which is based on electricity as a substance and on electro-dynamic energy, and which is pronouncedly speculative in character.

All phenomena of a mechanical nature can be reduced ultimately to modifications of inertia, velocity, momentum, and energy. Theories of mechanical action have, in addition, this common property; they begin with the postulate of an entity which possesses inertia and velocity. Various names have been at- 
tached to this entity, but whether it be called an atom, an ether, a vortex, electricity, or energy, its function is the same. While there is little difference in the nature of this entity or substance, yet there are two diametrically opposite ways of considering its space attributes which give rise to two irreconcilable schools of thought. One of these considers the primordial substance to be discontinuous with its parts separated by vacuous spaces, and the other school just as confidently assumes that there can be no empty spaces, and that all space is therefore continuous. Arguments for and against these two views have been unceasing and are likely to continue, so long as hypothetical methods remain in vogue. The problem is quite incapable of solution because the postulate of continuity or discontinuity is a matter of conviction rather than of reason. The fact is, we believe one or the other to be true and that is the end of it. In such discussions where a postulate is beyond our powers of verification by experience, each contestant chooses his startingpoint, and this once chosen, his argument in favor of it is as sound as that of his opponents. And the truth of this assertion is evident, because from these diametrically opposite postulates, conclusions which are quite identical are deduced by equally logical arguments; witness the controversy between the atomists and the Cartesians which see-saws through all scientific theory and is as far from settlement to-day as it was 
centuries ago. This dualism, as it may be called, is not confined to science; it is but one phase of that larger dualism of philosophy and ethics which has come down to us from the Greek thinkers; the claims of two opposing schools of thought are always present to show us that, if the mind can develop a system from one set of postulates, the same results can be obtained from their contraries.

If then our conception of the nature of things is but a matter of personal conviction, what value is there in the opinion of Professor Lorentz, which I have already quoted,-if we wish to obtain an insight into the mysteries and operations of nature, we must make hypotheses? If such hypotheses are not capable of proof then they must rest on what Bolingbroke so aptly calls our inward sentiment of knowledge. The experimentalist who described phenomena from his inward sentiment of what they should be rather than from observations of what they are, would be classed as a nuisance. Such a one not only does not advance our knowledge but he actually retards it, since his work must be repeated before the truth can be known. So, too, the theorist, who relies on his personal conviction and not on facts and laws, builds a structure which is not only temporary and false but must be torn down. If we relegate to metaphysics so large a part of what is commonly called physics, what is left to the science of physics? The answer probably 
is to be found in the saying of Lord Kelvin, that no scientific statement is understood until it is measured. If we grant this, then we must first examine, in such a discussion as this, our methods of measurement.

The statement of a phenomenon invariably contains two terms, called qualitative and quantitative factors; the former expressing "what kind" and the latter, "how much." Thus, when we speak of a distance as ten centimeters, we mean that we are to consider a quantity, ten, of the quality, length. Now it is not really the province of science to seek for absolute knowledge of either qualities or quantities, and the attempt to do so is the excuse for hypothesis. What science is concerned with is the relative knowledge, or comparison, of different quantities of any quality and the reduction of complex qualities into combinations of simpler ones.

It thus becomes of prime importance to settle on the simplest and most fundamental qualities which may serve as a foundation for our system of measurement. Because of the fact that mechanical motions and mechanical forces are the most readily perceived by us and are most easily expressed in mathematical formulæ, the fundamental units of quality are always selected from mechanical concepts. Of these, length, time, and mass or inertia, are found to be incapable of further simplification and have been adopted as the units of measurement. As these qualities must enter 
into all equations quantitatively and as we can have no conception of an absolute quantity, we have by statute fixed upon certain arbitrary standards of quantity, such as the centimeter and foot for length, the gramme and pound for mass, and the second for time. From these prime standards, all other quantities of a mechanical nature may be readily derived, as for example: velocity is the quotient of a given length by a time; momentum is the product of a mass and a velocity; energy, one-half the product of a mass and a square of a velocity, etc.

As I have already pointed out, the science of mechanics is the only branch of physics which has a completely developed theory. Not only is this the case, but the other branches of physics have been developed from a mechanical basis, in so far at least as the measurement of all phenomena is now made in terms of these mechanical units. This coördination in physics has the great advantage of making it possible to express what are apparently unrelated phenomena by using combinations of only three standards of measurement and by a few general equations of mechanics. And we attain the additional advantage of expressing all these phenomena in the most concrete form imaginable. But, on the other hand, this method produces a serious break between physics and certain other sciences, especially those which rely more exclusively on the sense perceptions as criteria for classification of 
phenomena. Thus, to the physicist light of different kinds is distinguished only by the three qualities possible in a mechanical wave; its wave-length, the amplitude of its disturbance, and the complexity of its form. These same qualities must also serve to distinguish sounds, water waves, and many other types of this kind of motion. For the biologist and psycholcgist, light is distinguished by its tint, intensity, and saturation, the three qualities which affect the sense of sight, and sound is determined by pitch, loudness, and timbre, which affect the auditory nerve. And as there is no relation between the sensations of sight and hearing, so there can be no connection between light and sound. While the method of the physicist has the great advantage of unity, yet it suffers from its artificiality, as it tends to bring into undue prominence the mechanical energy involved in producing light and sound and ignores the more important property of these phenomena: their effect on our senses.

It is generally conceded that we have no abstract or absolute knowledge of a quantity of length, time, or mass. In other words, we can express any of these three quantities only as a numerical ratio with respect to a predetermined standard of the same quantity, such as a yardstick for length, a pound for mass, and a given motion of a clock-hand or of the rotation of the earth for time. And, from this fact, it is often argued that we cannot form any idea of the qualities, 
space, time, and matter, except as they are individually and concretely measured. Kant, to be sure, maintained that we were endowed with an innate and inexplicable, but sufficient idea of pure space and time. These qualities are, however, by themselves inappreciable to our senses. To make them sensible, we need a third, which he calls the Ding an sich, corresponding in the external world to what we call the entity, matter.

However vulnerable and unsatisfactory the doctrine of innate ideas may be, yet it seems to me that by the abstractive method as used in the science of mechanics we do obtain a real and adequate idea of these three fundamental postulates. If we have not such a power of abstracting real ideas from our concrete observations of phenomena, then it is difficult to believe that the conclusions of Euclidean geometry and of mathematical analysis in general are rigorous. Thus, in mathematical definitions, space is a mere volume enclosed in an imagined boundary which may be conceived as of any extent from the indefinitely small to the indefinitely large. And this imagined bounding surface bears no closer relation to a concrete material envelope than does an image in a mirror to its object. It seems to me that I have an adequate idea, for instance, of the space in an empty room and that I can abstract all the properties from my concrete perception of the material walls of this room except the 
one fact that they inclose this space. By deduction and by experience I am convinced that this space is not empty, but contains air. But it is really more difficult to appreciate the existence of the air, if it be at rest, than its non-existence. This belief is borne out by the slow advance in the conception of the existence of gases. And it also seems true to me that I have an adequate idea of an area inclosed in a triangle, because I can think of the area inclosed by three abstract lines and refrain from thinking of the concrete volume of any real lines drawn with a pencil.

As for the concept time, we have a twofold sense of it; one is the coincidence of an event with the position of the hands of a clock or of the earth. But we have, in addition, a much more general idea of time, as mere succession of events without any reference to such a standard of measurement as the second or minute. This might be called our belief that events entirely unconnected with our own experience occur successively just as those events do which we compare with a clock. We have, on awaking from sleep, a distinct and clear idea that events have transpired successively during our unconsciousness and without any reference to a measured interval of time. This general knowledge of "before and after" is apparently possible to an animal; a dog will with certainty expect a reward after the performance of a trick and not before or during it. 
As for the third postulate, which is called matter, it may be taken in the most general sense as the something which makes space and time concrete or sensible. The necessity in science for such a postulate is clear, for science deals with the phenomena of an objective world. Even those men of science most opposed to the idea that mass is the measure of matter still postulate an entity identical with matter, as just defined, although they may call it by a different name, such as energy or electricity. And the distinction between mathematics and science lies in the conception of this third postulate. In pure mathematics, matter becomes the abstract postulate, quantity. When discussing velocity abstractly there is then no need to consider the properties of the thing moving, but in physics there is such a need. Or again, when dealing with space relations the mathematician is in no way limited to the restricted number of three dimensions which our sense perception of the material universe imposes on science.

The supreme value of mathematics to science is due to the fact that scientific laws and theories have their best, if not their only complete, expression in mathematical formulæ; and the degree of accuracy with which we can express scientific theory in mathematical terms is a measure of the state of a science. Thus it is possible to classify sciences according to their development, from the accumulation of statistics of 
phenomena to the generalization of these phenomena in comprehensive and rigorous laws. In such a classification, sociology or the study of existing society occupies the lowest rank, since true laws can be derived only from actions whose completed consequences are known. Sociology therefore attempts to found its laws on the data of history, the study of past society; history must in the same way rely on psychology, which deals with the actions of the individuals of society; psychology relies on biology; biology, on chemistry; chemistry, on physics; and physics, on pure mathematics. While each science thus strives to found its laws on the conclusions of the following science, each succeeds only partially; this leads us to a paradox. The goal of science is mathematics, and while mathematics may be said to be the only true science since it has the only true scientific method, mathematics is not a science because it deals with abstractions and ignores concrete phenomena.

As stated before, all quantities in mechanics may be expressed by combining the fundamental units, length, mass, and time, in simple ratios and products. And, while the phenomena of light, heat, and electricity do not manifest themselves qualitatively to our senses in any manner which may be coördinated with mechanical actions, yet we endeavor to measure them quantitatively in physics as if they were wholly mechanical in nature. Thus we speak of the velocity 
of light from the sun to the earth, as if we were considering a real mechanical motion of a ponderable body, although in the intervening space there is no matter. The only thing we have been able to observe is, that light emitted from the sun appears on the earth some time later. Light, as a phenomenon, does not exist unless it is associated with matter; we can no more discuss the amount of light or its velocity in vacuous space than we can speak of the temperature of such space. Light has a true mechanical velocity when passing through space occupied by matter, for then we can observe and measure its path as well as the time function and so obtain a value for velocity. From observations on the velocity of light in matter and from analogy to the phenomena of sound transmission, which takes place only in spaces occupied by matter, we by the hypothetical method transfer the measurements and laws of light in transparent bodies to space not occupied by matter. The very assumption of a light velocity in immaterial spaces requires us also tacitly to assume that something, in a mechanical sense, is moving. Once we have granted that light is something moving, then all the other phenomena of light permit of a hypothetical mechanical explanation, and we have the right to speak of the momentum and energy of this light something, whether it be corpuscles or waves. But it is the easiest thing in the world to forget that we can never obtain any real knowledge 
of this something we have called light. It is surprising that men of science should believe that they have proved the existence of the ether, as when Sir Oliver Lodge states that it is the most massive thing imaginable; or that space is occupied by energy, an entity possessing inertia and probably gravitational force, as Professor Einstein announces: they should see that such statements are not deductions made from our experimental knowledge of light, but are already contained in the postulate that light is mechanical and has a mechanical velocity.

It is instructive to consider, in this connection, how we also have attached hypothetically the science of electricity to mechanics. The fundamental phenomenon observed, when bodies are electrified or magnetized, is that they attract or repel each other with a mechanical force which, like the force of gravitation, varies inversely as the square of the distance between them. In our fundamental units this mechanical force is equal to a mass times a length and divided by the square of a time. Now Coulomb, who discovered and measured the law of electrical attraction, believed that electricity was a kind of fluid substance, such as was always introduced when phenomena were obscure; and with this idea in his mind, he employed the term quantity of electricity to indicate an analogy with a quantity of matter. On this supposition, a quantity of electricity expressed in mechanical units is equal to 
the square root of a length times a mass. Now it is quite certain that a quantity of electricity has nothing in common with length. Taking a step further, we find that the resistance of a conductor to an electrical current may be expressed as a velocity. Yet it would be absurd to attach any concrete relation between electrical resistance and mechanical velocity.

These three examples of velocity illustrate quite clearly the difference between the abstractive and hypothetical methods. From the definite and clear idea of the mechanical velocity of a concrete body, we pass to an indefinite idea of the velocity of light in empty space and in order to link this idea to mechanical notions, we assign to light a hypothetical material existence. But our mathematical equations lead us a step further and we can derive a purely formular relation between mechanical velocity and electrical resistance; in this case analogy between physics and mathematics entirely fails and no idea, even hypothetical, has been attached to the result.

The principles of the science of mechanics, on which the theories of the other branches have been built, date from the time of Galileo and Newton. As they had withstood the searching criticism of the masters of the science for centuries without having been shown to be either false or inadequate, the belief grew that however other theories of physics might change, the laws of mechanics as stated by Newton were prob- 
ably final. But it is inevitable that if we found the laws of light and electricity on mechanics, the time will come when the accumulation of knowledge will increase the discrepancies which must always exist between any two branches of science and which will eventually require a thorough revision of one or the other. If the attention be directed more toward discovering the phenomena and laws of light and electricity than of mechanics, as it is to-day, these discrepancies will probably be laid to the laws of mechanics and their revision will be attempted to insure agreement. This has occurred in the last few years, and the mechanics based on material bodies is being replaced by a mechanics of electricity or by one of energy, if the terms be permissible.

The mechanics of material bodies, to which the name of Newton is generally attached, was based on the objective reality of matter, whose quantitative measure was inertia or mass. Newton evidently considered inertia as a fundamental attribute of matter, and thus invariable and inexplicable; something to be accepted and determined solely by experiment. Thus he says in his Principia: "Haec (materiae vis) semper proportionalis est suo corpori, neque differt quicquam ab inertia massae, nisi in modo concipiendi. Per inertiam materiae fit, ut corpus omne de statu suo vel quiescendi vel movendi difficulter deterbetur." This postulate may be freely translated to mean that the force of attrac- 
tion of matter is always proportional to the amount of matter acting, and does not differ in any way from the inertia of mass except in our method of apprehending it. By inertia of matter is meant, that a body can be changed from its previous condition of rest or motion only by this material force. It is evident that he regarded inertia as an inherent and inalienable property of a body, independent of the influence of any other body or ether, and forming the connecting link between ourselves and the external world. Such being the case, how may we decide what is the mass of any particular body? If a number of individuals measure experimentally a mass, or even if one of them measures it several times, no two observations will agree. Which observation gives the correct value? Newton would have answered, none of them. Data of objective phenomena can never be known exactly; each value we obtain approximates to the truth, and the approximation is the closer, the greater the number so obtained and the greater the care exercised. The final result must be deduced from all the observations, according to a well developed mathematical theory of errors. The same reasoning was held to apply to observations on the space dimensions of a body and on the time occurring during any event.

While the idea was advanced in this mechanics that the position and motion of any body could be determined only from the position of some other body, 
supposed for the time being to be at rest, or that position and motion were relative and not absolute; yet it was not explicitly stated that there could not be absolute rest or motion, such as would occur if the motion of a body were referred to an absolutely fixed center of the universe or to an ether which was incapable of motion. For all practical problems, Newton's third law of motion, which states that to every action there is an equal and oppositely directed reaction, announces the universality of relativity.

We should finally note, that mass, dimensions, and time were held to be unaffected by the motion of a body. Newton expresses this by saying that force actions of matter, or the science of dynamics, are independent of its initial state of rest or motion.

The first serious criticism of these postulates was made by a number of physicists, forming what is often called the school of energetics, who proposed to substitute energy for mass as the fundamental attribute of matter. At the time, the change was rather immaterial as we were accustomed to think that mass and energy were coexistent and that either one was unintelligible without the other. As I have said before, it was the same kind of a problem as deciding which came first, the owl or the egg; the answer to which is we know nothing about the matter.

The discrepancies between electricity and mechanics did not prove to be embarrassing until certain problems 
connected with light and electricity became pressing. Of these, three stand out most prominently.

The phenomena associated with electricity when it passes through very high vacua and with radio-active bodies like radium are now explained as being due to the action of excessively small particles, carrying a charge of electricity and moving with a velocity comparable to the velocity of light, or about one hundred thousand miles per second. These particles, both because of their smallness and because of their velocity, are in an entirely different class from the bodies previously considered in mechanics which have a sensible mass and whose greatest velocity is less than one hundred miles per second. Errors in mechanical laws which would otherwise be inappreciable may easily assume large proportions when applied to such extreme cases. The hypothesis of the electron has profoundly modified the conception of the atom, and the nature of matter and motion.

In the second place, recent experiments lead to the belief that matter is always electrified; that light is due to electrical variations; and that the amount of electricity associated with a given amount of matter is a fixed and invariable quantity. It is further shown by theory and confirmed by experiment that if an electrified body be moved, the ratio of its electrical charge to its apparent mass must vary with the velocity of its motion; and since the electrical charge is assumed 
to be a constant, there remains only the alternative of considering the mass of a body as a variable. This evidently strikes at the very root of Newtonian mechanics. The effect of motion on mass is found to be inappreciable until the velocity approximates to that of light, so the discussion would have remained a purely academic one, if the creation of the electron had not brought us suddenly face to face with bodies which are supposed to have a velocity great enough to affect experimentally their mass. A further consequence of this theory is that mass becomes infinite when the velocity of light is reached. While that velocity has always been considered enormous and beyond our power to attain, such a result was entirely unsuspected. The laws of mechanics of bodies at rest could evidently no longer be held to be the same as those for bodies in motion.

Lastly, various experiments have been made to find an effect due to the mutual relations of the ether and matter. None could be found. One in particular, devised by Professors Michelson and Morley, has proved to be the hardest problem in modern physics to explain. Calculation showed that certain properties of light which depend on its velocity should be affected appreciably by the motion of the earth through space, but the experiment proved beyond doubt that such was not the case. The first attempt at a reconciliation was made by assigning certain complicated motions to the 
ether which would annul the effect the motion of the earth should produce; but it was shown that unless the ether remains absolutely at rest worse difficulties result. Then the bold assumption was made, often called the Fitzgerald-Lorentz effect, that the dimensions of bodies in motion were so changed by their motion as to neutralize the effect of the earth's motion on the phenomena of light. Every body would, according to this idea, grow shorter, the faster it moved; and would flatten out to a disc of no thickness if it could attain a speed equal to the velocity of light.

One of the results of this criticism of mechanics has been to change profoundly our hypothesis of the ether. As is well known, the early conception of the ether was a kind of material substance possessing properties incompatible with those of any other kind of matter. A large part of the effort of theorists down to the middle of the nineteenth century was devoted to inventing attributes for it which would enable it to fulfill its function as the medium for the transmission of light. At this time, Faraday discovered an effect in electricity which required an entire change in our ideas of the ether. When he found that static electric charges and forces were dependent on the characteristics of the material substance in which electrified bodies were immersed, and when later Maxwell predicted and Hertz showed experimentally that electro-magnetic energy passed through vacuous space, and that this form of 
radiant energy was undoubtedly of the same type as light and heat; then it was found that no material ether could be imagined which would perform these new duties, especially as it was already staggering under the burdens of the old ones. Faraday proposed as a substitute, that we should no longer imagine the medium to be a substance having material or mechanical properties, but one responding to electrical and magnetic actions. He was far in advance of his time and to be acceptable the hypothesis needed the mathematical development which was so skillfully accomplished by Maxwell. Both Faraday and Maxwell, while really destroying the material nature of the ether, strove to maintain at least a partial connection between electro-magnetic and mechanical attributes. For this reason they supposed that electro-magnetic stresses manifested themselves by creating mechanical strains in the ether and in matter immersed in it. Such strains must produce actual physical deformations of size and shape in all electrified bodies. It has been shown experimentally by the writer that such deformations are not produced in electrified matter and lately Professor Lorentz has stated, that since Helmholtz proved these stresses would cause the medium to move, and since no experiment has ever shown us a trace of a motion in the ether, we must deny the real existence of such stresses. The effect of such a denial is to separate all attributes of the ether from concrete realities and to class them 
as abstract symbols. Professor Lorentz is quite explicit on this point, as he holds that, " while thus denying the real existence of ether stresses, we can still avail ourselves of all the mathematical transformations by which the application of the formula (for these stresses) may be made easier: . . . and for convenience's sake we may continue to apply to the quantities occurring in this integral the name of stresses. Only, we must be aware that they are only imaginary ones, nothing else than auxiliary mathematical quantities."

This dematerialization of the ether, when it was once found that light and heat still managed to come to us from the sun although we had given its medium of transmission such a rude shock, has progressed rapidly until to-day many accept the postulate that there is no difference between absolutely vacuous space and the ether, except that the latter is the temporary seat of radiant energy and possesses a light vector. If this definition of space means anything, it implies that light has the power of changing a vacuum into a substance. When we stop to think that a vacuum means absolute negation of everything, we realize what an extraordinary thing light is in modern physics. Just consider this statement of Professor Einstein, which is considered almost authoritative, "the places in space where these electro-magnetic actions (i.e., light) occur are here considered not as states of a sort of matter, but as self-existing things which are similar to ponder- 
able matter and in common with it have the characteristic of inertia." One has merely to ask, what becomes of these self-existing things when light ceases to go through a certain space and it slips back into its state of vacuity?

Without going into details, we find a like trend in the hypothesis of the nature of matter. It began with the atom as a minute simulacrum of ponderable bodies, and then we proceeded to strip away one concrete attribute after another until for a brief interval matter was a manifestation of an entity, electricity. But even this idea was too concrete, too material, to serve and now the objective universe is the symbol, energy.

If we finally subscribe to these ideas, are we not really acknowledging that hypothetical science has failed as the interpreter of an objective world? It seems to me the guides to knowledge are now to be found in those subjective impressions which must depend on the individual and vary with him. Scientific laws are thus not facts to be discovered but the temporary consensus of opinion of a number of individuals who, for the time being, find themselves in agreement. The classical natural philosophy of Newton and Galileo has drifted into that transcendental symbolism which is apt to take place when German thinkers become the leaders in philosophy.

Evidently an almost chaotic condition had developed in our ideas of space, matter, and electricity. 
Each theorist advanced an hypothesis of the most tentative character which all felt to be inadequate. Yet, in spite of the confusion, three points of agreement may be noted. In the first place, the ether as a material substance is impossible and even useless; the name, it is true, is left but it expresses now but the shadow of a reality. When the ether is called an electro-magnetic something, or a space differentiated only by the transient presence of energy, the name may signify something but it is hardly intelligible except as a mathematical symbol. Secondly, these theories agree in assuming electricity to be an entity; that is, we are to suppose that it is not due to a state of matter, manifested by a special mechanical force, but a substance like matter, divisible into atomic elements and having inertia. Thirdly, the mass inertia of matter is not an invariable quantity but changes with the velocity of a body; thus it is the measure of matter only while there is no motion.

Such was the state of physical theory when Professor Einstein announced, in an article published in the Annalen der Physik for the year 1905, a new scientific principle which he believes will reconcile the contradictions of our new hypotheses; satisfy the three conceptions mentioned; and correct and amplify the Newtonian mechanics so that it will again harmonize with modern science. Whether this Principle of Relativity will accomplish all that its discoverer hopes can be 
decided only after an exhaustive trial. But there is no doubt as to the startling effect it has produced on scientific theory nor of the far-reaching importance of its conclusions. For example, Professor Planck of Berlin is said to have declared that this new idea of time and space surpasses in boldness anything that has appeared up to the present time in speculative science.

The name, Principle of Relativity, is derived from Professor Einstein's first postulate, which is as follows: the idea of absolute rest or absolute motion is an impossibility to the human mind, and not only in mechanics, but also in electro-dynamics corresponds to no properties of phenomena.

In addition he announced as a second postulate that the velocity of light, $\mathrm{V}$, in empty space is an absolute constant of nature. Hence this velocity, contrary to all others, is independent of the motion of the body emitting and of the one receiving the light. The startling nature of this postulate is readily understood when we remember that the closest analogue to light is sound, the velocity of which is known by experience to be dependent on these quantities.

He has recorded for us, that he became convinced of the necessity for these postulates, because the theory of electro-dynamics developed by Maxwell leads to an asymmetry, when applied to moving bodies, which is not true experimentally. For instance, when a magnet and an electric conductor are moved with respect 
to each other, the phenomena of the forces developed are observed to depend only on the relative motion of the magnet and the conductor, but Maxwell's theory requires a different explanation, according to which is moved and which remains at rest. Besides this discrepancy between observation and theory, experiments, devised with sufficient accuracy, fail to show any effect of the earth's motion through space on the phenomena of light.

It will be convenient to grant these postulates and follow Professor Einstein's deductions before attempting any criticism. In the first place, their adoption does away with the possibility of an ether and revives the postulate of space as a vacuum. Both theory and experiment show that an ether, if there be one, must be in absolute rest with respect to the motion of the earth, and the first postulate denies the possibility of absolute rest and motion. Again, the failure of Newtonian mechanics lies in the assumption, which is always tacitly made, that moving bodies are subject to the same mechanical laws as those at rest. As I have already pointed out, the modification to be made in the laws for ordinary bodies is very minute and would probably never have become of importance if modern theory had not been interested in the properties of bodies moving with velocities approximating that of light.

The root of the error in the mechanics of moving 
bodies, Professor Einstein believes, lies in our determination of time, and clear thinking in regard to time is nearly all that is necessary to clear up the trouble. Suppose a body or a material point to be at rest relatively to a coördinate system of three rectangular lines, then its position can easily be determined by the ordinary geometrical method of measuring its distance from each of the lines by rigid measuring-rods. But if the point is in motion with respect to the referencesystem, its position depends on time and cannot be determined by the geometrical method. Our idea of time is usually defined by what we call isochronism; we say an event occurs at seven o'clock, when the occurrence of the event and the position of the hour-hand of a clock at seven are simultaneous. But suppose the clock were at some distance from us, then we could observe the hand to be at seven only after it had passed beyond that figure, since it would take some time by any method of transmission for the intelligence to reach us. As the velocity of light is the greatest of all known motions, the least discrepancy would be caused by using light signals as the mode of transmitting such intelligence. Also, if we accept the second postulate, our intelligence will be still more accurate because the velocity of light is unaffected by other motions and we thus avoid the difficulty caused by the question whether any relative motion between us and the clock is an approach or a separation. To 
illustrate further our confusion as regards the measurement of time, suppose two persons wish to record two events, which we shall grant to be simultaneous, but which occur at different places, A and B. An observer at $A$ records the event, when it occurs at $A$, as being at $\mathrm{T}_{\mathrm{A}}$ time by his clock, and instantly signals the fact to an observer at $B$. Whatever the means of signaling, a certain time will elapse before the observer at B is cognizant of it. For the reasons given, we shall adopt light signals as the best method. B observes the signal as $T_{B}$ time by a clock placed at $\mathrm{B}$ and immediately reflects it back to $\mathrm{A}$, who receives it at $\mathrm{T}_{\mathbf{A}}^{\prime}$ time by his clock. Although we have granted that both events were simultaneous, it is evident they will not be so recorded by the two observers. B will record the event at $\mathrm{A}$ as being later than the one at $\mathrm{B}$. But if the velocity of light be absolutely independent of all conditions, then we should find that the differences of time going and returning are the same, or $T_{B}-T_{A}=T_{A}^{\prime}-T_{B}$. This relation is satisfied by any two events occurring at a distance from each other, is Professor Einstein's definition of simultaneous events or isochronism. The definition may be put in this form: two events are simultaneous, if the difference of time to flash a signal there and back is equal to twice the distance between the two positions divided by the velocity of light, $\mathrm{V}$.

Not only does this limitation in our ability to meas- 
ure time affect our ideas of time, but it also has an important and unsuspected influence on our ideas of the size and shape of a body. This may be shown by an example. Suppose we wish to measure a rigid rod of length, $l$, moving in the direction of its axis with a velocity, $v$, then there are two methods of measuring this length and they do not give concordant results.

First an observer may move with the rod and measure its length by applying directly to it a measuringrod. He will evidently obtain the same result as if both he and the rod were at rest.

A second method is possible and is frequently employed. An observer remains at rest and notes the positions of the two ends of the rod, at a certain time, $t$, which he determines by means of clocks, also at rest and tested for synchronism. These two points are dependent on our ability to record simultaneous events; if the rod were at rest the problem would be the one already discussed, but in this case the rod has a velocity, $v$, and consequently the time used in signaling in one direction involves, $\mathrm{V}-v$, and in the other, $\mathrm{V}+v$. So when he measures the distance between the two points, which may also be called the length of the rod, he will find it not equal to $l$ as determined by the first method, if he still regards his clocks as isochronous; or if he determined two positions whose measured distance is $l$ then his clocks will no longer be isochronous. 
Now our measurements of length are usually made under the condition that we, clocks, and rods are all moving with the earth and so relatively at rest. Our measurements of length of bodies on the earth are thus different from those which would be made on the same bodies by an observer if he could be stationed with his clocks in the moon. Also if an object on the earth is measured while it is moving with respect to the observer, it will appear to have a different length from that which it has when relatively at rest. Thus the dimensions of a body are dependent on its velocity, and Newtonian mechanics, which assumes the contrary, must be modified when applied to bodies in motion.

Professor Einstein then derives a set of equations which will express the dimensions of a moving body as they appear to a stationary observer. These show that the length of every body moving with a velocity, $v$, is diminished in the direction of its motion by the fraction

$$
1 / \sqrt{1-(v / V)^{2}}
$$

and that its dimensions at right angles to its motion remain unchanged. Thus a sphere in motion becomes an ellipsoid flattened in the direction of its motion by an amount equal to the above fraction. It is proper to say, that this effect is quite inappreciable at ordinary velocities. This is true even 
of so great a velocity as that of the earth around the sun. This velocity is about thirty kilometers per second while $\mathrm{V}$ is 300,000 kilometers per second. One diameter of the earth would thus, to an observer in the sun, appear shortened about 7.5 centimeters, or three inches. But this effect becomes quite important for velocities approaching one-tenth of V. And a velocity equal to that of light is absolutely unattainable because the length of the moving body would then be reduced to zero. So we have the curious anomaly of a finite velocity producing an infinite effect.

The same equations also show that if one of two clocks, which are synchronous when at rest, be moved with a velocity, $v$, the stationary clock will run faster each second than the moving one. The maximum value of the difference is when one clock runs infinitely faster than the other.

We must not lose sight of the fact that this discussion, so far as mechanical bodies and motions are concerned, is purely academic, because for them the ratio $v / \mathrm{V}$ is too small in any known case to have an appreciable effect. The Principle of Relativity has its greatest significance when applied to problems in electricity, provided we accept the theory of electrons. If the atom of matter be composed of particles of electricity, if the inertia of matter be variable and due to electrical reactions, and if the velocity of the electron 
approaches that of light, then the effect of its velocity on its shape, size, and mass is an important matter. For, let $m$ be the mass of an electron at rest, from Professor Einstein's formulæ, its apparent mass in the direction of motion is

$$
\frac{m}{\sqrt[3]{1-\left(\frac{v}{v}\right)^{2}}}
$$

and its mass at right angles to the motion which would make it resist change of direction is

$$
\frac{m}{1-\left(\frac{v}{v}\right)^{2}}
$$

Thus we have the unusual result of not only a variable mass, but also a difference in mass according to the direction considered.

Another consequence of the hypothesis is, that mass also depends on energy. This result is of extraordinary importance, for it means we have no ability to distinguish between the inertia of a physical system of bodies and its energy; in other words, between the inertia of a body and the energy content of the space surrounding it. When we recall Professor Einstein's conception of space and energy, which I have quoted, most persons will become convinced that no idea of the nature of matter could be more abstract. The mass of a single body thus remains constant only when 
its energy remains constant. If it gives out heat, light, or electro-magnetic energy, or if it even moves, its mass continually decreases, until, theoretically at least, it would melt away into a complex of energy: I know of no name to designate so immaterial a thing as this complex of energy located somewhere in vacuous space. To be sure, this interchange between mass and energy is very slow, so slow that ordinary minds grow skeptical of its existence. Thus a body radiating enough heat to warm a kilogram of water, one degree Centigrade, would decrease in mass only about $4.6 \times 10^{-11}$ grammes. It is fortunate that mass dissipates so slowly or we should indeed be things dreams are made of.

The last deduction I shall note is that the temperature of a moving body is less when measured by a moving observer than by one who is stationary.

Such are some of the more startling conclusions which have been derived from the Principle of Relativity. If they can be established, and men be persuaded that the universe is, or even may be, as they indicate, a revolution has been accomplished in scientific thought. If we are to criticise the conclusions of this hypothesis, and they are opposed to what seems reasonable, it must be done by examining the postulates; in them, as in all hypotheses, lies the real strength or weakness of the system.

Professor Einstein asks us to accept two postulates. 


\section{I74 THE LIMITATIONS OF SCIENCE}

The first is; we have no ability in us to determine absolute rest or motion. We can say only that one body is at rest or changes position with respect to others. Phenomena are conditioned entirely by the relative positions of bodies and we can gain nothing toward an explanation by introducing the idea of absolute position. The second postulate is; the velocity of light, $\mathrm{V}$, is a universal and absolute constant.

Apparently no objection has been made to his statement that these two postulates are sufficient from which to derive all the conclusions mentioned. But it seems to me, at least two more independent postulates are advanced in the memoir on relativity, which must also be granted. I shall propose as a third, Professor Einstein's definition of time, and as a fourth, the assumption of the atomic nature of electricity. The fourth postulate carries with it as corollaries, that the amount of electricity per atom, or the electron as it is called, is a universal constant, and that the mass of the electron is variable. My excuse for offering them is, they cannot be derived from his postulates, and his conclusions require them.

The first postulate can be granted at once and unreservedly for all purely mechanical motions of bodies. These involve merely changes of position and do not affect the nature of bodies nor their phenomena. But it must be accepted with limitations when in addition to mechanical motion, and by mechanical motion I 
mean a mere change of position, one body is emitting energy in the form of heat, light, or electricity, and the other receiving it. True relativity requires a strictly symmetrical arrangement. Now the mechanical motions of two bodies with respect to one another are symmetrical. If a body has a velocity, $v_{\mathbf{1}}$, to the right and another a velocity, $v_{2}$, to the left, they approach each other with a velocity, $v_{1}+v_{2}$. The same result is obtained if the velocities of the bodies are interchanged. This symmetry is not true if the first body is emitting energy and the second receiving it. The behavior of each is then conditioned by the behavior of the other and in an asymmetrical manner. As an illustration: let a body by a periodic disturbance of its parts send out a wave of heat; this proceeds, according to theory, through space in all directions and on encountering a second body produces a periodic disturbance in it. The effect of this energy is twofold; the motions of the parts of the two bodies are symmetrical but the wave itself does not proceed from the second body in all directions but only in the one already impressed upon it by the first body. To make this clearer, we may start a wave along a stretch of string by plucking aside one part of it; the wave will travel from the initial point along the string in two directions, causing successive parts to vibrate symmetrically, but these parts will send the wave in one direction only. So we may say the velocities of two bodies are relative because the 


\section{THE LIMITATIONS OF SCIENCE}

quantities appear in the mathematical expression in a symmetrical manner; so also are their momenta or their masses multiplied by their velocities. But this is not the case for energy since it involves the square of the velocity. The squared velocity remains positive although we change direction and the composition of two energies is always an addition. For true relativity, a change in direction must be accompanied by a change in sign. The extension of the idea of relativity to involve cases of radiant energy is partly responsible for the conclusion that mass is a function of energy.

Professor Einstein's second postulate has been widely discussed and many attempts have been made to interpret it. At first sight it seems absurd to say that the mechanical motion of a body emitting light does not affect the velocity of the light emitted. But the fact remains that all our experiments, and they have been most accurate and searching, fail to find any difference in the velocity of light whether the sources and the recipient are at rest or in motion. As we have seen, Professor Einstein, with admirable directness, goes straight to the point: he accepts the reliability of these experiments; assumes the constancy of $\mathrm{V}$ as a postulate, determines its effect on the space dimensions of bodies and on time, and finds that we must change our previous ideas of these radically. Let us, when discussing $\mathrm{V}$, consider it under two heảds: first, when light passes through transparent material 
bodies and is subject to experimental verification; secondly, when it passes through space absolutely deprived of material bodies and so is not subject to experimental evidence.

In the first case, we know that there is a true path and that light moves very approximately in straight lines, and we have quite accurately measured the time light requires to move from one place to another through various substances. $\mathrm{V}$ is here the length of path divided by the time, - a true velocity. We know that this,$V$ is a variable; it is less in water than it is in air, and still less in glass. It is also less in dense air than in rarefied air. Not only does $V$ vary with the kind of matter through which light passes, but it also depends on the motion of the medium as shown in the experiments on the velocity of light passing through columns of moving water made by Fizeau and repeated by Professor Michelson. The velocity of light in material media is therefore subject to all the variations which influence the velocity of sound and other types of motion and evidently cannot be the $\mathrm{V}$ assumed by Professor Einstein to be an absolute constant.

He must then limit his postulate strictly to what is called the velocity of light in absolutely immaterial space. There are two methods of obtaining this value. We observe the difference in time between the calculated eclipse of some satellite and the recorded observation of the event or we use other stellar phe- 
nomena. If we know the distance and the time, we say the velocity of light in empty space is the quotient of the two. Such observations are quite inadequate to settle experimentally the question of the constancy of V. Our measurements of the distances are crude for such a purpose, and also the light must travel part of the way through a material medium such as the air. Neglecting such astronomical methods, we have left only determinations of $\mathrm{V}$ made on the earth where the path is necessarily through matter. Here, what we are to call the constant $\mathrm{V}$ can be found only by extrapolation. For example, we find that the ratio of the velocity in air and in water is about four to three and agrees with their refractive indices. We find also that the velocity in different gases as they are reduced in density tends to a common value, which is independent of the kind of gas and approaches the value $3 \times 10^{10}$ centimeters. If we take this extrapolated value of $3 \times 10^{10}$ centimeters for the velocity of light in empty space and assume the refractive index of space to be one, then we may calculate back and by this method we find the absolute refractive index of air at ordinary pressure to be 1.0002 . Such an experimental method and such reasoning are highly unsatisfactory. In the first place, we start with measurements of lengths and time which are subject to experimental verification and calculate $V$, from these we pass to a supposititious $V$ where only one factor, the time, is subject to experi- 
mental proof and the other factor, the length, is not, as we cannot experiment on the path of light in free space. But passing this objection, we have calculated from length and time determinations a value for $\mathrm{V}$ and Professor Einstein declares it to be an absolute constant. $\mathrm{He}$ then reasons backward that the length of a body and time determinations must be variables with motion, in order to keep V constant. Of course, if Professor Einstein wishes to look on the universe as a purely abstract conception; and if he wishes to make what we sometimes call concrete or objective phenomena correspond to his preconceived idea of the universe, he can assume $\mathrm{V}$ in a vacuum to be a universal and absolute constant and no one can disprove it scientifically. But there are some who still cling to the idea that dimensions of bodies and time are not subject to our fancy, and who believe that if any quantities must vary, it should be those which we have no means of determining directly. And after all it is asking a great deal of us, to upset our ideas in order to explain at bottom a single experiment, that of Michelson and Morley, however accurately it has been performed and however puzzling its results may be. $\mathrm{He}$ has not even the justification his predecessors would have had. When the ether was believed to be a crystalline solid which vibrated with the passage of light rays, $V$ had a real meaning although we could not determine it directly. 
When the ether was a substance which periodically varied electro-magnetically, we could still say that $\mathrm{V}$ had a possible meaning. But the latest definition, that the ether is absolutely quiescent space to be distinguished from vacuous space only by the fact that it is the seat of an entity, called electro-magnetic energy, and contains a light vector, makes the word velocity when applied to $\mathrm{V}$ absolutely without meaning in any ordinary sense of the term. To speak of a motion in an absolutely quiescent space is a rather startling statement. The ether also becomes a local affair, shifting back and forth in vacuous space according as light is present or not. The feeble light of a candle, apparently, changes nothing into something.

But aside from these considerations, which lie in the debatable land and which will be decided largely by the temperament of the individual, there seems to be an absolute contradiction between the first two postulates as I understand them. If $\mathrm{V}$ be the value of the velocity of light in an absolutely quiescent ether and itself a constant, then the velocity of light in a material medium, such as air, is an absolute velocity or motion when referred to $V$. Now the first postulate declares that we can have no knowledge of absolute motion and, in addition, that the explanation of phenomena is not conditioned by absolute motion.

The third postulate, which I have proposed as necessary for the system proposed by Professor Einstein, 
is his definition of time. He says,-our idea of time is defined by synchronism or the simultaneous occurrence of an event and the position of the hour-hand of a clock at a certain position, the number seven for example. This is certainly not our idea of time but merely our method of measuring it quantitatively. If we did not have an adequate idea of time as the mere succession of events, we should have no conception of what simultaneous occurrences are nor of how to measure time. His definition gives me the feeling that if I could make clocks go slower my life would become longer. In the review of his memoir, it was pointed out that his definition of simultaneity or isochronism, when combined with the constancy of $\mathrm{V}$, led to the conclusion that two clocks which were synchronous, when relatively at rest, would not remain so, if one of them were given a velocity. Moreover the length of a body in motion decreases with respect to its length when at rest. It is certain that these results depend on the constancy of V. If we are willing to rest our knowledge of time and space on a value which by the nature of things can never be put to a direct test, I am convinced of the theoretical correctness of these results. But I am not willing to do this. I prefer to trust to the invariability of time and space phenomena, even if it requires $\mathrm{V}$ to be a variable and time measurements to contain an unavoidable discrepancy. By so doing, I am aware that I forfeit an 
explanation of the Michelson-Morley effect and of some others, but this loss is not overwhelming and we can afford to wait for an explanation until a future time.

I am the more willing to take this attitude, because I can see no means of applying the Principle of Relativity to the test of experience. As we have seen, the velocity of light in any material medium is a variable and can be no more trusted than the velocity of sound.

Perhaps this illustration may make my meaning clearer. Suppose a race of men to exist who are blind and have no knowledge of electro-magnetic radiation, but who wish to measure the lengths of moving bodies. They will undoubtedly be compelled to get this information and that of the synchronism of clocks by sound signals. It is evident that observations carried out under conditions similar to those imposed by Professor Einstein would indicate that the length of a moving body underwent changes. And while they could make corrections for some of the effects, because sound waves are largely affected by the motion of media and of sonorous bodies, yet they would undoubtedly come to the conclusion that the dimensions of a moving body depended to some extent on its motion. Now, if we should bestow sight on one of these men, he would be able to correct their measurements; as he could by his immensely more rapid light signals gain a much more nearly instantaneous value for synchronism. 
We are, at present, in the condition of this man. As we improve in our ability to measure the velocity of light under different conditions we shall, Professor Einstein thinks, get closer to the knowledge of the absolute $\mathrm{V}$ and to the relations for space and time which he has derived. But we may suppose men will some day find a kind of radiation which has a velocity greater than $\mathrm{V}$ (for example, the transmission of gravitation), and by its aid remove the conviction remaining in our minds that motion affects length and time. Calculation may show that material bodies cannot attain this velocity, but we are speaking of an immaterial radiation. To say that such a radiation is impossible is as futile, at least as unscientific, as for a race of the blind to say that there is no light.

Since the motion of any ponderable body is too slow to make the ratio $\%$ an appreciable quantity, the only supposable case, where this ratio can enter as a determining factor, is in problems of radio-activity and the discharge of electricity through gases. In these, the particles of matter are supposed to be so small and to have a velocity so great that their mass and size are measurable functions of their velocity. But to limit the applicability of the principle of relativity to such supposititious bodies as electrons, is to rob it of its importance, and we should hardly consider it one of the great principles of nature.

But even this is not all: if we wish to apply the 
principle to electrons, we shall need a fourth postulate. We must assume that a quantity of electricity is atomic in character; either the charge of electricity associated with what we call an atom of matter is an invariable, or, as it is now usually expressed, electricity is an entity which may be divided into invariable, equal, and indivisible parts called electrons. As a corollary to this postulate, the ponderable mass of an atom is a variable depending on its velocity. It has been shown that the constancy of electric quantity and the variability of mass is not a necessary assumption. All experiments, which involve both these quantities, include them in the form of a simple ratio, $\% \mathrm{~m}$; where $e$ is the quantity of electricity and $m$ the mass of the electron. It is evident that any value may be given to this ratio by supposing either one of its members to remain constant and the other to vary, or by supposing both to vary in opposite fashions, and it is just as reasonable to keep $m$ constant and to let $e$ vary with the velocity, as to make the contrary assumption. This is certainly possible until we have experimental evidence which will determine our decision, and this evidence is not likely ever to be at our disposal.

If we attempt to estimate the results which follow from the postulates of relativity, the first would be that Professor Einstein has proved that we cannot theoretically measure in space and time moving bodies exactly by the laws which apply to bodies at rest. The first 
postulate will be accepted for all mechanical motions and no further attempts should be made to find experimentally an absolute motion. But the second postulate contradicts the principle of relativity, if by $\mathrm{V}$ we mean the absolute motion of something,-matter, energy, or light through quiescent space. If, on the other hand, $\mathrm{V}$ is understood to be the velocity of light in space containing matter, then it cannot be taken as a universal constant. The third postulate concerning time should not cause us to change our belief that the dimensions of a body and the unit of time are independent of velocity, but it should show us how to correct our measurements of moving bodies, as we must correct all subjective measurements of objective phenomena.

If we grant the assumptions of Professor Einstein, the theory of relativity is a perfectly logical system so long as we apply it to abstract systems moving with a constant velocity in a straight line. But I can find no evidence that the postulates agree with experimental facts and certainly, to the present time at least, its conclusions will not affect experimentally the laws of mechanics. Also its theoretical conclusions are limited, as yet, to the rare cases when there is neither any change of speed nor of direction. We should remember before we abandon or modify Newtonian mechanics that mathematicians have devised numerous systems which point to different mechanical laws. If we pos- 
I86 THE LIMITATIONS OF SCIENCE

tulate a fourth dimension to space we can deduce perfectly logical laws for a mechanical system that are quite different from the laws of ordinary mechanics. And the universe, constructed on this principle, is a very beautiful and interesting one. It is the privilege of mathematicians to deal with symbols; the physical universe is no more important to them than any other universe which can be developed symbolically. The trouble occurs when the distinction between the real or physical universe and symbolical or metaphysical universes is obliterated in the minds of men of science. 


\section{CHAPTER VI}

\section{SKEPTICISM AND IDOLATRY IN SCIENCE}

E egli questo un far loro oracolo una statua di legno, e a quella correr per i responsi, quella temere, quella riverire, quella adorare.-GALILEO.

THE intellectual habits of men have a very decided effect on their characters, and it is evident that Galileo had met with contemporaries who carried their devotion to the hypothetical and dogmatic scientific method of Aristotle to a state of idolatry, accepting on faith that which should be submitted to experience. The quotation, from his Dialogues on the Two Principal Systems, which I have given expresses his opposition to such idolatry in no uncertain language. It is fortunate for me that I wrote this chapter as an essay with the same title before I had read Galileo's comment, because the quotation is so ben trovato that otherwise I should have been accused of fitting an argument to a title.

Men of science have two principal functions to perform: first to observe the phenomena of the world; and when certain connections and differences are found in these phenomena, to classify them under laws. The conclusions, thus derived, have been used with great success to enlarge our intellectual life; to modify 
our environment; and to promote our comfort and power. But, allured by their great and legitimate success in these two fields of activity, they have also tried to discover the hidden causes of phenomena, with the result that a sort of fictitious world has been created by them, in which the laws of objective, or physical, phenomena are inextricably confounded with the deductions of subjective psychology. Science is made metaphysical, and at the same time pretends to supplant metaphysics.

This encroachment, naturally, has not been accomplished without a sharp conflict, and the history of the nineteenth century is permeated with the struggle of science against religion and philosophy. The issue was clearly in favor of science, which has not only gradually become a dominating influence in education and in thought, but has also changed our attitude towards history, politics, and social life to such an extent that these subjects are now classed as sciences. Even philosophy and religion were unsettled by its growing power; instead of basing character on attributes peculiar to man and designated by the name of spiritual powers, many now include man with the rest of organic life as subject only to the statistical and impersonal laws of heredity and environment: metaphysics tends towards a philosophy of science; religion to eugenics.

Both the conflict and the victory were no doubt inevitable. But the results have not been on the whole 
beneficial either to society or to science. It would be difficult to prove that the acceptance of the belief that man has no divinity, in at least the sense of supernatural powers, and no innate standards of right and wrong-in other words, that he is merely the most complex machine in a world governed exclusively by physical and chemical laws-that such a belief has not sunk him to a lower plane of morality and induced in him a resigned weariness towards such a fate. The victory has not only reacted on science in such a way as to give the world a too implicit confidence in its hypotheses, but it has also made men of science dogmatic and rash in proposing bold and unsupported speculations,-impatient of criticism of themselves and hypercritical towards religion, philosophy, and all other methods of human thought. Thus the most urgent need is a severe and just criticism, not of our experimental observations or of our logic, but of our hypotheses. One of the greatest difficulties in developing such a criticism lies in the fact that so few men of science leave any record of their intimate and personal convictions as to the limitations and the possibilities of their field of action. They content themselves, for the most part, with a mere statement of experimental results, or they develop speculative theories without much consideration whether they are fanciful or whether their influence will be good or pernicious. Such criticism as there may be is directed 
towards checking the accuracy of observation and the logic of deduction. As for hypothesis, there is a generally accepted and naive belief that any such endeavor is beneficial.

The writings of men of genius in science frequently exhibit this sort of naiveté in method of thought. And probably a too critical attitude would be a hindrance to their creative powers as they must look at problems intensively, and exclude the many complexities and doubts which would tend to embarrass them in their special work. It would be foolish to say "thus and thus only shall a man work out his ideas." We have an Ampère who develops the laws of electro-dynamics from the experimental facts of electricity, and quite independently of the prevailing hypothesis of the nature of electricity; and, on the other hand, a Lord Kelvin who could not work until he had first visualized a problem, and usually he put this image into a concrete form, making for the purpose many different models of atoms out of sticks and strings, to illustrate the properties of matter.

But science has other functions in addition to the attack and solution of problems by the creative power of the individual: phenomena and laws must be taught in detail to the specialist; general conclusions must be given to the world in order that they may become a part of the general intellectual life; and finally discoveries are to be applied to social and industrial needs. 
Thus, not only the results obtained by creative minds, but also the methods of obtaining them, are important, because they will ultimately be interpreted and promulgated to others. The harm done by a vicious method of thought may outweigh the benefits of a useful discovery happened upon in spite of them. There is always this danger in the hypothetical method; the man who assists his intellectual labors by the help of crude mechanical models undoubtedly tries to keep distinct in his mind the real actions of nature from the properties of his model, let us say of the atom. But in imparting his results to others, this model is made to assume an aspect of reality in the written word which was not in his own mind. Baffled by the diffculty of expressing complicated ideas, he paints his metaphorical pictures too vividly. The process grows more pronounced as the idea passes from mind to mind; the fictitious model grows progressively more and more concrete, until to the student and to the world, it is at last the concrete model of the atom that becomes the reality, while real matter dissolves into an abstraction. And if words can be relied on to express ideas, the creator of the atom himself is a believer in the reality of that creation of his imagination, the model.

This sort of speculation is probably least dangerous in pure mathematics. The mathematician looks upon the world symbolically. He studies its laws from pos- 
tulates which are given to him by others, or assumed by himself. Thus he may derive the laws of mechanics from a postulate that bodies have three spatial dimensions, which is verified by observation; but he is also interested in the mechanics of bodies which would prevail if there were four dimensions to space, a postulate not verifiable. He develops a geometry which requires the axiom that parallel lines do not intersect; and at the same time he studies another geometry based on the axiom that parallel lines do intersect. He finds it no more important as an exercise in analysis to study a solar system which is subservient to forces of attraction, than one which obeys a law of repulsion. That is, he is not limited at all to what we call the reality of an objective and material world, and mathematical deductions regarding an imaginative world will have very little influence on society. So, also, the purely hypothetical parts of such abstract subjects as physics, chemistry, and astronomy are not very influential in a direct manner, but indirectly they have had an enormous influence since they have been taken as an example for the development of biology and that class of sciences known as political and social. Speculations in these subjects have a direct and intimate bearing on the character of the individual and on society. Thus a ready proneness to accept hypothesis and speculation as well as observation has resulted in the crude laws and dogmas of eugenics, and has put 
government under the necessity of hearkening to the dilettante of the slums, and the feminist; all of whom are preaching the scientific basis of their nostrums. It is not then a matter of indifference what the hypotheses of even the abstract sciences may be, or how we attempt to solve their problems.

Two books* have recently appeared most opportunely, which record the mature beliefs of two conspicuous men of science concerning the value and necessity of scientific hypothesis. We could scarcely have two authors more widely contrasted in temperament and in opinions than Poincaré and Sir Oliver Lodge; the one, a skeptic who views with detachment the efforts of men to penetrate the mysteries of nature, and the other, a credulous believer with equal conviction in matter and ether and spirits.

Before discussing their views, we should call to mind that Poincaré began his career and attained his greatest eminence as a pure mathematician; that he. later critically examined the theories of physics; and finally turned to philosophy for the purpose of examining the foundations of science. Sir Oliver Lodge has

* The Foundations of Science. By H. Poincaré. An authorized translation by George Bruce Halsted of "Science and Hypothesis," "The Value of 'Science," and "Science and Method," with an introduction by Professor Royce. (The Science Press.)

Continuity. The Presidential Address to the British Association for the Advancement of Science for I9I3. (Putnam's.) By Sir Oliver Lodge. 
worked mostly from the experimental side. With an early tendency toward speculation, he wrote several books which aimed to give a most concrete, and even crude, picture, of natural phenomena. His present position as executive head of a university has thrown him into the practical affairs of life. And probably his chief trait of mind is the belief that all things, from engines to souls, are best considered as manifestations of a luminiferous ether which has the characteristics of a jelly.

It will be best now to let Poincaré state his ideas regarding the scientific method in his own words: for he has done so most lucidly and vividly. The quotations are from Professor Halsted's authorized translations:

"Experiment is the sole source of truth. It alone can teach us anything new; it alone can give us certainty. But then, if experiment is everything, what place will remain for mathematical physics? What has experimental physics to do with such an aid, one which seems useless and perhaps even dangerous? And yet mathematical physics exists, and has done unquestionable service. We have here a fact that must be explained. The explanation is that merely to observe is not enough. We must use our observations, and to do that we must generalize. This is what men have always done; only as the memory of past errors has made them more and more careful, they have 
observed more and more, and generalized less and less."

"It is often said experiments must be made without a preconceived idea. That is impossible. Not only would it make all experiment barren, but that would be attempted which could not be done. Everyone carries in his own mind his own conception of the world, of which he cannot so easily rid himself. We must, for instance, use language; and our language is made up only of preconceived ideas and cannot be otherwise. Only these are unconscious preconceived ideas, a thousand times more dangerous than the others."

And he goes on to show how barren would be the mere accumulation of experimental facts, and how the master minds group them into generalizations and laws which make them fit for use. Out of complexity they achieve simplicity and order. And he warns us that even in this true phase of science, lurk the dangers of preconceived ideas, and of inaccuracy of expression. Having pointed out the proper path, he then enters the more debatable field of hypothesis:

"All generalization," he says, "is a hypothesis. Hypothesis, then, has a necessary rôle that no one has ever contested. Only, it ought always, as soon as possible and as often as possible, to be subjected to verification. And of course if it does not stand this test it ought to be abandoned without reserve. This is what 
I96 THE LIMITATIONS OF SCIENCE

we generally do, but sometimes with rather an ill humor."

"The firm determination to submit to experiment is not enough; there are still dangerous hypotheses; first, and above all, those which are tacit and unconscious. Since we make them without knowing it, we are powerless to abandon them. Here again, then, is a service that mathematical physics can render us. By the precision that is characteristic of it, it compels us to formulate all the hypotheses that we should make without it, but unconsciously."

It is just here that I think Poincaré begins to leave sure ground, and by a confusion of thought and terms he falls into what seems to me an impossible position. He first stated that a generalization is an hypothesis. It is true that every generalization is speculative to the extent that we cannot verify it by experiment with absolute accuracy or in all possible cases. For instance, no one supposes we can ever record all the changes of energy which occur in the universe, yet we state with great confidence that energy is conservative. The justification for our belief is simple. All the cases we have measured confirm the law. And this law is of the kind that can be frequently and readily put to the test of experimental verification; until future experiments shall show it to be in error, we have the right to believe the law correct. But hypothesis has a wide range of meanings besides that of 
signifying a tentative generalization which will ultimately be accepted as a law or be rejected because of its increasing disagreement with observations and, unfortunately, it is used very loosely. It frequently means those speculations in which we indulge when we attempt to define the causes of phenomena; when we create fictitious substances, as an atom or ether; and when we construct models to illustrate the actions of forces. None of these cases is a generalization in the sense of Poincaré's first statement, because it cannot be verified by experiment. It would greatly simplify thought if we would confine the term hypothesis to these speculative ideas, and use the words law or theory for those generalizations which are based on experimental observations of a material world. One has only to note Poincaré's own confusion of thought; if we must generalize, and if our generalizations are hypotheses which are subject to, and inust be verified by, experiment, then such hypotheses are the truth, so far as can be determined by scientific methods. We certainly have the right to ask: how can they be dangerous and how can there be a too great multiplication of them, if they are the truth? If they can be subjected to experiment, then as soon as one is disproved, it would necessarily be abandoned. The reason for his caution to us is that Poincare knows that the great majority are incapable of verification, since they deal with purely metaphysical ideas. His own words, 


\section{THE LIMITATIONS OF SCIENCE}

quoted from the introduction to his Théorie de la Lumière, will prove this:

"It matters little to us whether the ether really exists; that is the business of the metaphysician to find out; the essential thing for us is that everything acts as if it existed, and that this hypothesis is convenient in explaining phenomena. After all, have we any other reason for believing in the existence of material objects? Is not that belief also a convenient hypothesis; only we shall never cease to make it, meanwhile the time will come, without doubt, when the ether will be rejected as useless."

See what an advance in confusion of thought we have now! Hypothesis is no longer a necessity, it is a convenience. If those of the ether (and no idea has been more fruitful than the ether) cannot be verified by experiment, how can they be convenient, because he says unverifiable hypotheses are dangerous; and if they can be verified, how can we reject the truth, whether they are convenient or not? Again, how can everything act as if the ether existed, unless it does exist? Is not that our definition of existence? And lastly, one would suppose that if we shall never cease to postulate the existence of matter, we have come as near making matter a certainty as we can ever attain to any certainty.

The unfortunate feature of such statements is that they constantly rise to trouble their makers. We shall 
see later that Poincaré absolutely reverses this opinion, rejects matter and clings to the ether; meanwhile, both of his opinions are unsupported by experimental evidence, which he has declared to be the touchstone of truth and usefulness for hypotheses. Not only does Poincaré drift in this hopeless fashion between matter and ether, but the founders of the New Mechanics are at sea as regards the existence of the ether. One school of thought claims that all substance is but a differentiation of the ether, which thus becomes universal substance; the other school is just as positive in its belief that the ether is a wornout fiction. In spite of this trifling difference in their points of view, both schools arrive at much the same conclusions. At least they give us a good illustration of the power of mind to modify matter. During the recent conference at Brussels for the purpose of discussing the new concepts of matter and mechanics, Poincaré suggested that it was unfortunate that physicists were using the ether when they needed it, and were discarding it when it proved troublesome. Curiously, no one in the conference seemed to find the situation of the New Mechanics in the least embarrassing or humorous; the fact is, the consequences announced at the conference were so unintelligible that a sense of humor would have been out of place.

Although Poincaré says that we must generalize, and that every generalization is an hypothesis, still 
he finds he must distinguish between different kinds, and must warn us against their indiscriminate use: "It is important not to multiply hypotheses beyond measure, and to make them only one after the other. If we construct a theory based on a number of hypotheses, and if experiment condemns it, which of our premises is it necessary to change? It will be impossible to know. And inversely, if the experiment succeeds, shall we believe that we have demonstrated all the hypotheses at once?"

This is excellent advice, but when he makes hypotheses, he does not heed his own warning and neither does anyone else. Imagine a physicist saying to another, yours is dead, now let me have a turn with one. But let us follow Poincaré's classification further:

"There are first those which are perfectly natural and from which one can scarcely escape. It is difficult not to suppose that the influence of bodies very remote is quite negligible. . . They are the last that ought to be abandoned.

"There is a second class of hypotheses, that I shall term neutral. In most questions the analyst assumes at the beginning of his calculations either that matter is continuous or, on the contrary, that it is formed of atoms. He might have made the opposite assumption without changing his results. He would only have had more trouble to obtain them; that is all. If, then, 
experiment confirms his conclusions, will he think that he has demonstrated, for instance, the real existence of atoms?"

"These neutral hypotheses are never dangerous, if only their character is not misunderstood. They may be useful, either as devices for computation, or to aid our understanding by concrete images, to fix our ideas as the saying is. There is, then, no occasion to exclude them.

"The hypotheses of the third class are the real generalizations. They are the ones that experiment must confirm or invalidate. Whether verified or condemned, they will always be fruitful. But for the reasons that I have set forth, they will only be fruitful if they are not too numerous."

It would seem to almost anyone that the first and third classes are so different from the second class, that it is advisable to call them laws, and limit the term hypothesis to the speculative second class. The class of neutral hypotheses is evidently the melting-pot for the bewildering ethers, atoms, subtile fluids which abound in the physical sciences, and which have their analogues in all the others. Poincaré teaches us that we shall never acquire any definite knowledge from them, and then he makes the amazing statement that there is no need to exclude them if they are not multiplied, if they are made one after the other, and if their character is not misunderstood. Anyone in the. 
least conversant with their history knows that their number is great, and that they are not proposed consecutively. As for their character, he evidently means that they are dangerous if we forget they are mere conveniences, fictions, not to be taken too seriously. It is perfectly safe to say that there is scarcely a textbook or a treatise in any science which does not state explicitly that the sort of things classed by Poincaré as neutral hypotheses, are realities and not conjectures. Before showing how Poincaré and Sir Oliver Lodge confirm this opinion, let me quote two statements of Sir J. J. Thomson:

"The ether is not a fantastic creation of the speculative philosopher; it is as essential to us as the air we breathe. . . . The study of this all-pervading substance is perhaps the most fascinating and important duty of the physicist."

"The possession of a charge by the ions increases so much the ease with which they can be traced and their properties studied that, as the reader will see, we know far more about the ion than we do about the uncharged molecule."

Evidently convenience of computation and an aid to our understanding by concrete images are not at all in the minds of even eminent physicists when they indulge in neutral hypotheses of ethers and atoms.

Now let us return to Poincaré, and see how he heeds his own warning. But first notice how clear and how 
vivid his thought is when he is not involved in these pitfalls to accurate reasoning: "Is not each great advance accomplished precisely the day someone has discovered under the complex aggregate shown by our senses something far more simple, not even resembling it-as when Newton replaced Kepler's three laws by the simple law of gravitation, which was something simpler, equivalent, yet unlike?"

To this excellent question, he gives the following lamentable answer:

"One is justified in asking if we are not on the eve of just such a revolution or one even more important. Matter seems on the point of losing its mass, its solidest attribute, and resolving itself into electrons. Mechanics must then give place to a broader conception which will explain it, but which it will not explain. . . . The ether it is, the unknown, which explains matter, the known; matter is incapable of explaining the ether."

How can Poincaré justify himself? When we recall how Newton refrained from giving any hypothesis regarding the cause or nature of gravitational force, because he felt such an hypothesis would be outside the field of science; and how the subsequent verification of the law of gravitation by innumerable experimental observations has established it as one of the few great and universal laws; and when we recall what Poincaré says about electrons, the ether, and 
neutral hypotheses in general, how can we be expected to see any similarity in these two revolutions? The two, on the contrary, are diametrically opposed to each other. Does Poincaré really mean that it is to be the function of science to resolve matter, which he said we must always assume to be an objective reality whether we can prove it or not, into electrons, an hypothetical component of the hypothetical atom, the assumption of whose reality should be a mere fugitive matter of convenience? And does he believe the boast of science, that it shall rest on the objective experience of our sensations rather than on the subjective proof of our imaginations, can be maintained, if we admit for an axiom, that henceforth it shall be the aim of science to explain the known by the unknown? $\mathrm{He}$ might have pursued this revolution further; the dissolution of matter into the electron is but the first step in the confusion of ideas, those who are promoting it are further transforming the electron into a strain in the ether of a type unknown to experience, and the most advanced are dissolving the ether into nothing. The process of explaining the known by the unknown is complete; matter to atoms; atoms to electrons; electrons to ether; ether to nothing. Truly, philosophy and theology are gross materialism compared with such a science. Can he so quickly and completely forget his wholesome, skeptical, and critical attitude, merely because he also is tempted to indulge in specu- 
lation? As an example of what absurdities are advanced in speculative physics, I give his final utterance on the existence and nature of matter, which he delivered in a lecture on the "New Mechanics": "We can almost say that there is no longer matter, but only holes in the ether; and in so far as these holes seem to play an active part, it consists in the inability of these holes to change their location without influencing the surrounding ether which exerts a reactive influence on such changes."

What to make of such a statement passes understanding. There was once a man who pestered the students in Baltimore by giving them tracts which proved in fifty-seven or more different ways that the earth was hollow, and that we lived on the inside, and so in a hole. But not even he was so confused as to contend that we were holes living on the outside of a hole. Poincaré has, of course, no resemblance to that man and yet, unless he means something by the word "hole" which is understood exclusively by himself and a small coterie of physicists who write in the same absurd fashion, there is no more sense in his definition of matter. If there were any connection between the words and the idea-that is, if he were trying to define the word matter so as to express our experience of it, then his statement makes matter the answer to the old conundrum: "What is it, the more you take from it, the more it be?" I admit matter is not ether; 
but if it is a hole, then a hole in the ether is just the contrary to a hole. Holes never even seem to play an active part in anything; they show no inclination or ability of themselves to change their location; and nothing, except an ether, has ever been conceived of as able to react on a hole. The simple fact of the case is, such statements are attempts to do just what Poincaré said they were,-attempts to explain the known by the unknown. The pity of it is that science is filled with just such attempts; we recognize the words used, but their sense is so twisted that they really express no clear idea. In comparison with such quibbles and such verbal distortion, the action of Dr. Johnson, when, in answer to the similar logic of a metaphysician of the Berkeleian type, he kicked a stone as our ultimate proof of the existence of matter, is true scienceat least it is organized common sense. It is comical to read the opinions, concerning the essence of matter, of those modern physicists who have succumbed to the fascination of explaining the known by the unknown. Led by a small band of German physicists, they use mathematical symbols and scientific phrases; and yet they are explaining phenomena in quite the approved medieval fashion of transcendental symbolism. It is perfectly easy to match their statements with the abstract theorems of an Albert Magnus or a St. Thomas Aquinas. In other words, modern science has suddenly taken a leap into pure metaphysics, while, at the 
same time, it persists in the habit of sneering at metaphysical methods.

It was with the greatest regret that I found Poincare had given in to this form of idolatry of the graven images of science, His mathematical writings, his physical theories, and his critical spirit had always impressed me as models of clear and vivid thinking. And the statements I have quoted show that he did not adopt this other mode of messy thinking without reluctance. His ideas no longer are unqualified: he is willing to admit only that things seem to point this way or that, as if he had been caught and entangled in this German school of transcendental symbolism, and could not see his way out.

Sir Oliver Lodge has no such scruples. He rejoices in his beliefs, and delights in promulgating a clear knowledge of all the unknowable things in the universe just as he sees them; especially by popular essays addressed to those whose lack of technical training makes them unable to discriminate between real and sham science. In his recent presidential address on "Continuity," he tries to accept at once the modern mechanics and Newtonian mechanics, continuity and discontinuity, and scientific laws for bodies both mortal and immortal. But with all the trouble which this reconciling of irreconcilables gives him, he always finds comfort and refreshment in his "fervent belief in 
208 THE LIMITATIONS OF SCIENCE

the Ether": in some way it will solve our difficulties. This is his creed:

"The Ether is the universal connecting medium which binds the universe together, and makes it a coherent whole [not hole] instead of a chaotic collection of independent isolated fragments. It is the vehicle of transmission of all manner of force, from gravitation down to cohesion and chemical affinity; it is therefore the store-house of potential."

In short, the Ether, not being matter, is for Sir Oliver pretty nearly everything else; almost, if not quite, God, even to the use of the reverential capital initial. More specifically, he considers it the principle of continuity, and in continuity he finds a scientific proof of immortality. If he had based a belief in the immortality of the soul on an analogy with the continuity of existence of matter and energy, I think many persons would agree that he was expressing one of our most certain reasons for such a hope. It is difficult to suppose that the quantity of matter and energy in the universe is unchangeable, and yet to maintain that thought and emotion, which have so many of the characteristics of durability, end with death. On the contrary, "we * do see the sum of mind increasing every time two old thoughts coalesce into a new one, or even every time matter assumes a new form before a receiving intelligence." But such an intimation of im- 
mortality is a very different thing from assuming a body of postulates and creating a de facto science of psychic phenomena, as Sir Oliver Lodge does. Science must deal with material things, and must be confined to our sense perceptions. So that, whether immortality and psychic phenomena be ultimately accepted or not, the proof must be expected by other than scientific methods, unless we give to these words a totally different significance from what they had in the past. Omitting all other difficulties, one wonders how immaterial intelligences can make material noises, for, if they do, what becomes of our law of conservation of energy which accounts for all material motion by material causes? Of course he falls back on his omnipotent Ether:

"The evidence to my mind goes to prove that discarnate intelligences, under certain conditions, may interact with us on the material side, thus indirectly coming within our scientific ken; and that gradually we may hope to attain some understanding of the nature of a larger, perhaps ethereal, existence, and of the conditions regulating intercourse across the chasm."

Science is still burdened with the inexplicable mystery of the material world, and it should not open the doors of its temple for the worship of graven images; even if the idol is the great god Ether. Sir Oliver Lodge's speculations are not even qualified by logical methods. His reasoning is apparently a mere play on 
words: Immaterial spirits are not material bodies, the ether is not matter; therefore spirits may be ethereal bodies. It does considerable harm when he uses his official position and, as it were, makes the British Association indorse such foolish views.

Sir Oliver Lodge still believes with the past generation of physicists that science has discovered a path to positive knowledge: "Many scientific men," he says, "still feel in pugnacious mood towards Theology, because of the exaggerated dogmatism which our predecessors encountered and overcame in the past. They had to struggle for freedom to find truth in their own way; but the struggle was a deplorable necessity, and has left some evil effects." It has left evil effects, and the chief of them is Scientific Dogmatism.

In spite of our self-confidence, we cannot be too sure that hypothetical science has not traversed the same path as dogmatic theology. We have a fairly large and clearly defined body of moral facts. And we have generalized from them rather consistent moral laws. It is inevitable that we shall always speculate as to the causes of these moral facts and laws, and it is probable that most men will continue to fashion an image of their idea of God, more or less anthropomorphic and concrete. But unfortunately for religion, many will not stop at this point of pure speculation, or the worship of God in spirit and in truth, but they will carve for themselves images in wood and stone, and 
bow down and worship these images as realities. So also there are the many who will not stop at the great and scientific work of discovering phenomena and laws, but they will speculate on natural causes and mechanisms. If they do this with an open mind, perhaps no harm is done as Poincaré says, and possibly the imagination may be healthfully stimulated. How few stop at this point may be imagined from the examples I have given; a large and increasing number have become idolaters, and are worshiping the graven images of science with dogmatic fervor. 


\section{CHAPTER VII}

\section{SCIENCE AS THE ARBITER OF ETHICS}

Sire, in this system there is no need of a God.-LAPLACE:

WHEN Renan wrote that humanity must, in future, look to science for what it craves in the way of a law and symbol, science was in the full swing of conquest. The war between it and religion was believed to be all but over. The law of evolution was accepted not only in its general aspects, but also it was believed that in natural selection a cause had been discovered which would permit us to trace the progress of the organic world from the simple protoplasm to the most complex forms of life. Search was made for the missing link between man and the lower animals, and those at-. tributes which an earlier age was content to group as spiritual powers were abolished as unscientific. It was inevitable that philosophers like Renan and Spencer would propose an evolutionary law for society, and that even religion would fall in line and make of God a sort of omnipotent and beneficent natural force which gently led man up to ever higher types of perfection.

And while to-day those who cultivate the more exact sciences have come to believe that the objective and 
subjective worlds are governed by different forces, the wave of evolutionary philosophy, under scientific domination, has spread to the students of political and social history who are trying to devise a state which shall evolve toward better conditions by natural means.

The result is that the attitude of thoughtful people toward the moral questions of the day is one of more or less perplexity. Few of them are willing to accept the doctrine that human nature is essentially good and that the only need of society is to remove all restraint. In the past, the majority of men experienced this necessary restraint in the conviction of personal responsibility to a God, who actively interfered in their thoughts and deeds, and who rewarded or punished according to a revealed standard of righteousness. But with the growth of knowledge and especially with the impersonal attitude assumed in scientific inquiry, the impression has become widespread that mankind has been left to work out its own destiny. So, as I have said, thoughtful people are perplexed to know where to turn to find an inner check which can replace that sense of personal responsibility to a God which, however men might rebel, did exert a steady and potent influence upon them. Some still cling to the idea that the Christian church will ultimately discover again the guiding principle for society; that a new reformation from within is all that is necessary. But the difficulty is graver than in the past, as such reformations 
involved matters of doctrine rather than a change in fundamental ideas. Many are simply drifting; but an increasingly large number are frankly turning to scientific doctrines in the hope that a deeper experimental knowledge of the laws of man's individuality, of his social relations, and of his environment will bring with it the solution of the problem. And in spite of our very deficient acquaintance with such laws, some are proclaiming that this naturalism is the surest guide for us in evolving a society nearer to the ideally good. Apparently, they have the idea that accurate observations and statistical records, which may be formulated into scientific laws, are sufficient as guides to conduct.

A curious feature of the discussions, which have arisen about these new systems of scientific ethics, is that almost no attention is paid to the fundamental question whether the deductions of science are suitable to serve as an ethical principle for society; or even whether science, except indirectly, concerns itself with character; yet character, or the judging between right and wrong, is necessarily the essence of all systems of ethics. Now I think it can be shown that the pursuit of science has for its aim to acquire power; that is, we seek through science to learn the facts of nature and to interfere with natural forces so as to make them serve man's desires. As an illustration of the mixture of truth and fallacy, which is typical of many who advo- 
cate scientific ethics, consider this statement of a writer on genetics which merely reflects a widespread opinion: "Human civilization goes hand in hand with the degree of successful interference which man exerts upon the natural forces surrounding him." The truth of this statement lies in the correct view that the purpose of scientific inquiry is not only to discover the forces of nature but to interfere with them so as to increase man's dominating power; the falsehood consists in assuming that civilization is based on, or even necessarily runs with power. Everyone knows that power may make for evil as efficiently as for good; the ethical standard of a civilization depends on the will of its individuals to choose between right and wrong. To deny this, is to refuse the attributes of the noblest civilization to Jesus, to Buddha, to Socrates, each of whom was quite ignorant of science, and lived in times when men exerted themselves but little to interfere with natural forces. Nor could we to-day, in the midst of an earnest scientific endeavor, deny that a civilization higher than our own would be possessed by a community formed of men like to them.

The greatest difficulty encountered, if the attempt be made to determine the ethical value of science, is, as I have said before, the lack of a spirit of serious criticism amongst men of science, with the consequent absence of critical data on the aims of science and on the effect which scientific activity has had on society. 
Before much can be done to find out what the influence of science on morals has been, a school of critics of men of science must be created. I am quite aware that this opinion is counter to the general belief that science is the most critical of all human endeavor. The results of experimental investigation and the mathematical laws formulated from them seem so positive, so subject to minute and patient scrutiny, that it may well seem that men of science are critical. But this form of criticism is limited to the mere question of accuracy of observation and logic. Before the subjective influence of science on character can be determined, the postulates of science and the scientific method itself must be outlined clearly and discussed critically; it is this higher criticism which is lacking in men of science.

If such a body of criticism existed it is probable that a philosophy of science could be created. But the present method of educating men to engage in scientific work is little likely to foster the critical or even the historical attitude. In fact, most men of science know so little of metaphysics or even of the historical development of science that they are quite ignorant of how metaphysical their sciences are. If they were conversant with the history of scientific thought, they would see that while scientific laws are as positive and as objective as human knowledge has been able to attain, yet the hypotheses of science, which aim to dis- 
cover the causes of phenomena and to simulate the mechanism of natural forces, are as subjective and as dependent on human opinion as any other form of knowledge. And it is just these subjective and metaphysical aspects of science which are concerned in the question of the relation of the external world of phenomena to our internal world of ideas.

One would naturally turn to the histories of science, not only for the data of scientific experience, but also for that larger aspect of the question, the discussion of the relations between scientific thought and other forms of human activity. But almost without exception our histories of science are mere chronicles of scientific experiments and hypotheses. The work of each man of science is outlined in as great detail as the scope of the history permits, as if everything done in the name of science were important. What we should have, is a discussion of the development of the subject and of the relations between the intellectual forces of a given epoch and the scientific activity of the same period; for example, there should be shown the relation of the ideas of Galileo, Copernicus, and Descartes to the Renaissance; at a later date there is a similar close connection between the rise of electricity, the skeptical spirit of the mathematical physicists, and the revolutionary and democratic ideas which were prevalent at the beginning of the nineteenth century.

The most striking evidence of the ineffectiveness 
of scientific histories is, however, their attitude toward hypothesis. Such hypotheses are clearly subjective in character and are to be criticised in accordance with the same standards as a philosophical system of Plato or Kant. Instead of this they are invariably treated as if they dealt with phenomena verifiable by experiment.

In our histories of science a number of things about scientific hypotheses might have been brought out. In the first place, all of them rest on mechanical analogies and attempt to make of the world, both inorganic and organic, a vast machine whose parts grind out motions which reveal themselves to us as heat, light, thought, and whatever else we are cognizant of. That is, a mechanical explanation of phenomena is considered to be the simplest and most satisfactory one that can be given. But we know there is no criterion which will distinguish the truth between many rival mechanical hypotheses. Thus the explanation of phenomena is dependent on subjective interpretation, and is in the end a matter of personal opinion. Nor does the recent attempt to replace matter by electricity escape this difficulty since the principles governing electrical forces and energy are the same as those controlling matter. The restrictions pointed out are still in force, with the substitution of the word electricity for matter.

It is also evident that, however logically a scientific hypothesis may be developed, we can never say that 
it is anything more than our idea of the world; thus scientific hypothesis stands on the same ground as any other kind of logical thought. Criticism of hypotheses should therefore be directed principally to their postulates.

In the next place, the postulates of science are always metaphysical in character since we must assume initial conditions of space, time, and substance which are not subject to experimental verification and which are contradictory to our experience of the present state of those quantities.

We imagine a simple primordial universe which is crudely fictitious, and start it going on its complicated career by the action of some universal force which unbalances and unlocks its internal actions and reactions.

Then we assume the existence of fictitious substances, atoms, which we deliberately fashion so small as to be below the limit of observation. We endue them with as many different and contradictory attributes as may suit our convenience in order to fashion the diverse forms of bodies by their combination.

And lastly, if we are to attempt to include in the name of science all laws, we must provide portions of this machine with life, thought, and self-consciousness as a form of mechanical forces.

Evidently, the postulates of science are as complex, 
as subjective, and as debatable as the postulates of religion and philosophy. And they also lack the simplicity and generality which have made the postulates of geometry universally acceptable and have given to them the appearance of finality. And from these metaphysical postulates of natural science, the scientific conclusions follow which somehow impress us as being positive knowledge not subject to human opinion.

Since most of our scientific postulates and the hypotheses which are developed from them are not questions of objective facts, they must have an influence which may be helpful, harmful, or indifferent. And it should be the chief duty of the historian and the critic to trace this influence. Instead of this critical spirit, it is only too common for men of science to insist that all hypotheses are meritorious. This opinion is so widespread, that it is frequently stated that even if a hypothesis be entirely false, yet it may be useful. The reason for this lack of discrimination lies probably in the fact that science has gradually created two worlds connected by only a slender thread; one the experimental world of our sensations, and the other a fictitious world of our imagination. Whatever the prevailing hypothesis may be, the experimentalist continues his work, confident in the belief that if his results agree with a hypothesis they will be taken as a confirmation of it; and if they disagree, then he may be sure that the hypothesis will be modified so 
as to conform to them. Thus the phenomena and laws of light continue to unfold themselves in a consistent progress in spite of the fact that now one, and now another, contradictory hypothesis of the mechanism of light and its transmission through space is uppermost. This is the explanation of the reason, so puzzling to the layman, why two conflicting hypotheses, explaining the same class of phenomena, can be maintained and cultivated at the same time. There is no telling when new facts will be discovered which will bring an almost discarded hypothesis back into favor. The fictitious worlds of natural science bear a close analogy to those hypergeometrical realms of the mathematician which, while logical and interesting, do not rest on experience and so do not exist. Such mathematical worlds of the imagination are invented as a sort of intellectual game and can have no influence on human actions and society; so also the hypothetical properties ascribed to nature by the physicist cannot affect our relations to our environment.

This neglect of the critical field by men of science has resulted in leaving to metaphysicians the discussion of scientific methods and the development of what now passes under the name of scientific philosophy. Whatever the influence of this criticism may have been on philosophy and on thought generally, it is safe to say that it has had little on science; its conclusions have not engaged the attention of men of science very 
seriously. The reason for their indifference is twofold. Rightly or wrongly, it is felt that the metaphysicists are too engrossed in the exact definitions of terms and in the niceties of verbal expression. They thus miss or confuse the real issue of science, which is to discover the significance and influence of objective facts rather than of words. This does not mean that the careful use of words is not important in science. It is only too apparent that many of the battles of science are waged over the meaning of words and not of phenomena.

The other reason for the ineffectiveness of this scientific philosophy as developed by the metaphysicists is that they are not disciplined by labor in the laboratory nor trained in the analysis and technique of scientific theory. They must thus depend on elementary treatises, which are rarely the work of master minds; or they can approach the great thinkers only through the medium of popular expositions in which the most scrupulous are somewhat relaxed. To contrast the opinions of Plato with Ganot does not command respect; and to depend on the popular addresses of a Helmholtz or a Lord Kelvin is not to know their real thought. The first leads to frequent errors and the second to sweeping conclusions. If we are to develop a satisfactory scientific philosophy it seems requisite to have it done by men trained in science and conversant with philosophy; this combination has been 
a rare one, of which the greatest examples are Aristotle, Descartes, and possibly Kant.

It is difficult to see how this combination of science and philosophy is to be obtained with the present system of training men in our graduate schools. The prospective man of science is taught laboratory methods and becomes, thanks to it and to his research work, a rather skillful manipulator of apparatus, but he rarely is required to trace back the gradual development of the subject on which he is working. It remains in his mind as a more or less isolated fragment, since he is quite ignorant of the work of the master minds of science of the past. When he leaves the school, he is impressed by the head of his department with the opinion that he must continue his research work; left to his own devices he casts about for new problems, and, in despair of ideas, continues some of the minor points left unsolved in his thesis. A second article appears and then the curtain drops. In addition to his laboratory work, the student attends lectures in theoretical science and passes rigid examinations which are for the most part exercises in ingenuity in mathematical and logical exposition of the most modern and abstruse parts of the science. The lectures treat the science as a modern and fully developed theory devoid of continuity of background. Hypothesis is mixed with fact, and fugitive speculations with permanent laws; his texts are largely confined to purely specula- 
tive philosophy and fail to contrast the limitations and the permanent achievements of science; no connection with past thought is drawn and the probable aspects of future thought are not shown. Indeed, no mention is made of the prime fact that human thought has been concerned with these fundamental problems for so many centuries, and methods of scientific attack are so limited in number, that no new theory or hypothesis can be developed; witness our present return to Cartesianism. At most, what we call modern thought is but a recrudescence of past thought dressed in new clothing. The see-saw of the time-worn antinomies constantly recurs. Since the historical and critical attitude toward science is almost wholly neglected, the student enters his life work innocent of the limitations and powers of his science; practiced in modern textbooks and ignorant of the masters of the subject. Some teachers of science realize this vaguely and advise their students to take a minor in philosophy. Now the philosophers, not knowing science, have introduced certain emasculated courses under the name of scientific philosophy or scientific methods for just such students. One has merely to read the texts used and learn the views of the expositors to find that the texts are written, and the courses delivered, by men who know nothing of science at first hand. Thus the course merely discourages the student and gives him a contempt for philosophy as a juggling of words and quite 
different from the exact and virile sciences. If students of science are ever to attain to the wide outlook which the dignity of science deserves, teachers of science must themselves first learn the historical and critical development of their subject, establish its relation to other knowledge, and then impart this spirit to their neophytes.

If the postulates of science are doubtful and contradictory, what is likely to be the effect on science and thought in general of the hypotheses derived from them? The very general belief that any hypothesis, whether false or not, is useful to science puts us in a curious attitude in respect to other methods of thought. Evidently, it is a doctrine which does not hold elsewhere; the theologian, the philosopher, or the historian who ventured the same opinion would quickly be attacked; and yet science is supposed to be the search for objective truth. The reason for this peculiar attitude of men of science is due to the fact that the real work in science is connected with phenomena and laws, and this true aim of science has never really been lost sight of, however much the hypothetical method may have distorted it.

I suppose it may be granted that hypotheses should be divided into the three general classes of the good, the false, and the indifferent.

Good hypotheses are those which are known as laws. They are generalizations from such necessary postu- 
lates as the conservation of matter and energy, or they are advanced as tentative laws when a certain number of phenomena can conveniently be classified; they become steadily more exact as our knowledge of the subject increases and are finally accepted as laws; such has been the history of the second law of thermodynamics, of the law of evolution, and numerous others. The difference between these proper hypotheses or laws and other hypotheses is that a law does not attempt to explain the mechanism of nature as an hypothesis does. That is, laws may be readily recognized because they deal only with sensible matter and its attributes and can thus be subjected to a rigid test of their truth; all other hypotheses, since they attempt to explain natural actions, must create fictitious substances and attributes and when they are found to depart from facts they are not discarded but are merely modified by arbitrarily altering the fictitious substances on which they depend. This difference is shown clearly by Darwin's law of evolution and his hypotheses of natural selection and pangenesis, or by Newton's law of gravitation and his hypothesis of light corpuscles. The history of science points to no more certain conclusion than that laws persist and hypotheses decay. For this reason, I have emphasized the distinction between the two and have limited the term, hypothesis, to those scientific theories which require the creation of fictitious substances and occult forces and whose use has caused 
us to cross those boundaries which properly limit science.

As for false hypotheses, it would seem to be selfevident that they should be abandoned as soon as a postulate or conclusion was shown to be wrong. It is just as important to discard error in science as it is in anything else. And it is besides an added and confusing burden to the student to keep these false hypotheses in treatises on science; they should be relegated to histories of criticism whose chief purpose is to show the progress of science in its devious path toward the truth.

Unfortunately, most hypotheses cannot be readily classed as either true or false since they involve postulates which can neither be denied nor affirmed from scientific criteria; thus in themselves they are fitly classed as indifferent. Yet they may indirectly either benefit or obstruct the progress of science. So long as we keep clearly in our minds and in our statements the fact that such problems as a nebular hypothesis, the condition of prehistoric life, radiation in interstellar space, the ultimate constitution of matter, etc., are pure speculations, I do not suppose any serious harm is done. But the hypotheses which we have created with respect to atoms, the ether, natural selection, mutations, and the like, have a much more subtile influence, and they have been used in such a fashion as to confuse knowledge. 
Indifferent hypotheses will have a pernicious influence if by their use the deductions of science become increasingly complex. Since hypotheses deal with confessedly fictitious substances, it seems rather futile to create things which add to the difficulty of a problem.

Again we find our hypotheses are becoming so abstruse that they require a prolonged technical training and specialization before they can be understood; thus the science itself is restricted to a few specialists and loses much power. These abstruse hypotheses are producing a breach even between theoretical and experimental science. So marked is the difference between these two aspects of the same science that they have little in common and are frequently hostile.

But the most inevitable and dangerous influence of the free use of indifferent hypotheses is the breaking down of the scientific method by carrying science into fields where it has no business to be. I have tried to show what the limitations of science are and why the obliteration of its boundaries is prejudicial to it. In what has gone before, the discussion has been limited to excursions into what may be called pseudo-science. Encouraged by lack of criticism, there is now a desire to go much further and to claim that science is the sovereign of all knowledge. Thus, the example given in the last chapter of Sir Oliver Lodge's attempt to include the phenomena of immortality in the field of science is undoubtedly made possible by the steady. 
and gradual trend toward treating matter symbolically. The step between considering matter as tangible reality and as Poincaré's hole in the ether is really greater than passing from material to immaterial phenomena or than maintaining that science is the guide of character and the arbiter of ethics. In this way scientific naturalism is more dangerous than the easily refuted outbreak of Sir Oliver Lodge.

Any system of ethics must aim to develop character; it must establish a standard of good and evil; it must judge actions according to this standard and provide an inner check which will restrain the will of the individual. But scientific experimentation and theory do not directly consider character at all. The attempt is there made to discover objective facts and laws which have no character in themselves and to manipulate or interfere with natural forces so that they will add to our knowledge and power. No one would hesitate to say that the discovery of the laws of heat and their application to steam power were problems definitely in the field of scientific investigation or that they had added enormously to our power. They have in fact changed civilization, and yet we cannot deduce from science whether or not this acquisition of power has been accompanied by a beneficial effect on civilization. That can be determined only by the moral effect of the expansion of industrialism on the inner life and 
thoughts of men, and science has no criteria for these. The man of science is thus trained to discover new things. He may point out their dangers and advantages but the restrictions to be placed upon their use is not his affair.

If we were to trace the growth of the idea that the scientific method is our best means of obtaining information not only of objective facts but also of the motives of human thought and character, it would require a detailed history of science. But without undertaking so laborious a task, certain prominent factors in the rise of science can be indicated. In the first place we may assert that the guide of science during the early and medieval periods was the Organon of Aristotle. And we can also state pretty accurately that the revolution in science against the Aristotelian domination was accomplished under the leadership of Albert of Saxony, Leonardo da Vinci, Copernicus, Galileo, and Descartes.

The aim of these revolutionists was to replace the metaphysical method of Aristotle by the physical or experimental method. To put the issue simply, the purpose of the Aristotelian philosophers was to form a comprehensive and logical system of the universe as they conceived it should be, a philosophy in which experimental observation and inductive reasoning played but a minor rôle; the scientific method of the new school aimed to determine the laws of a universe 
which would be in accord with the phenomena discovered by experiment. Naturally the division was not sharp; even to Galileo, and especially to Descartes, much of the Aristotelian method still clung.

Just as the Organon of Aristotle absorbed the scientific spirit of the Greeks, so we have in the Novum Organum of Bacon an explicit attempt to crystallize the new knowledge into a scientific method. To make his purpose quite clear he invented for it a new name, natural philosophy, so as to separate it from the metaphysical philosophy of the Greeks. This natural philosophy, if carried out logically, would limit us to the laborious and careful accumulation of experimental facts, out of which would grow true natural laws. And, further to show the break he would make with the Peripatetic school, he states that any further generalization which would lead to the consideration of formal and final causes must require a hypothetical method proper only to metaphysics. His criticism of Greek philosophy is characteristic of his attitude. Thus he gives his well-known opinion that the Greeks were a vain and disputatious people, whose desire to shine, whose taste for dispute, and whose mania for new systems of thought multiplied error, leading them to forsake observation for the more facile triumphs of speculation. Nor did he spare the greatest, when he declared that Plato subordinated the world to ideas; and Aristotle, ideas to words. The one corrupted sci- 
ence by theology and the other by dialectics, as later Proclus did by mathematics. And while we have learned by experience that scientific laws are not to be discovered by such a regulated system of induction because science does not rest on a single broad foundation which rises uniformly, like an Egyptian pyramid, to a point that signifies a single dominant force, yet Bacon's inductive philosophy is still our authoritative scientific method. In this opinion we have the unqualified statements of such men as Newton, Huygens, and Laplace. Thus Laplace expresses most clearly what men of science even now generally admit: " $\mathrm{La}$ méthode la plus sûre qui puisse nous guider dans la recherche de la vérité consiste à s'élever par induction des phénomènes aux lois et des lois aux forces. Les lois sont les rapports qui lient entre eux les phénomènes particuliers: quand elles ont fait connâitre le principe général des forces dont elles dérivent, on le vérifie soit par des expériences directes, lorsque cela est possible, soit en examinant s'il satisfait aux phénomènes connus; et si par une rigoureuse analyse, on les voit tous découler de ce principe, jusque dans leur moindres détails, si d'ailleurs ils sont très-variés et très-nombreux, la science alors acquiert le plus haut degré de certitude et de perfection qu'elle puisse atteindre. Telle est devenue l'astronomie par la découverte de la pesanteur universelle."

It is natural that, if observation is the only sure 
guide to natural philosophy and if knowledge must finally rest on the sensations, Bacon would be considered by many as the founder of the doctrine elaborated by Locke, Hobbes, Hume, and others that there is nothing in the intelligence which has not previously been a matter of the sensations. But as de Rémusat* points out: "To say that in life all knowledge is derived only from the data of experience, and to say that everything which is in the intellect has been a matter of the senses, is to say two immensely different things. And Bacon has not said the second of these two things; he has not even said absolutely the first; he admits inspired knowledge." That is, Bacon apparently divided knowledge into classes, the physical and the metaphysical, and based the former only on the sensations. In confirmation of this opinion Bacon took a positive stand against atheism: "No one denies the existence of the gods, except him to whom it is serviceable that the gods do not exist. To deny God, is to destroy the nobility of the human race."

There seems then good reason for considering the Novum Organum as the starting-point of modern science, as in it is developed the doctrine which still prevails, that science must depend on observation, and that scientific theory must conform to the data of experience. 
While it is true that Bacon maintained a distinction between those things which are proper for scientific elucidation and those things which are not; and although he avoided a universal philosophy of the sensations; yet his predilection for the scientific method and his inclusion in it of such subjects as history, which in spite of time and effort has made little progress as a science, pointed the road to that philosophy. So also some believe him to be the originator of the idea of applying the scientific method to society and ethics. Such does not seem to be correct and I think it is not possible, because the rule of science over life could not have been accomplished until a great accumulation of scientific observations had been made. No such accumulation had been undertaken before the last century. In Bacon's time only a few enlightened minds were convinced of the need of systematic experimental knowledge. Even the British school of philosophers, known as rationalists, materialists, or atheists, who were more or less followers or supposed to be followers of Bacon, was a school of metaphysicians and not of scientists.

Of these philosophers, Hobbes undoubtedly had more of the scientific closeness of reasoning than the others. He based all knowledge on the sensations and in so far seems to exalt science to be the arbiter of life, but nothing could be less scientific than the postulates and the conclusions of his philosophy. He had little reali- 
zation of, and much contempt for, experimental knowledge. He assumed certain abstract definitions as universal postulates and from them derived all his conclusions by pure ratiocination without any regard as to whether these conclusions conformed to objective facts. A brief glance at some of the definitions and deductions, which may be found in his Leviathan, will show that he was advancing a purely metaphysical system of thought. Thus, every part of the universe is body. Bodies comprise those things which are formed by nature and commonly called bodies and those formed by the human will which we call societies; this latter idea still lingers in the phrase, the body politic. Motion is the cause of all things, even of the human passions, etc. The small beginnings of motion within the body of man before they appear in walking, speaking, striking, and other visible actions, are commonly called endeavor. Endeavor, when it is toward some thing which causes it, is called appetite, or desire. He distinguishes in the following and entirely arbitrary fashion between the sensations. Heat is motion resulting from the light endeavor; hearing is generated by the motion of the medium, but not in the same manner as seeing. Sight is an endeavor from pressure produced by the luminous body on the retina of the eye, and by the pressure on that part it will be propagated to the heart, the innermost organ of sight and of all the senses; and from the reaction of the heart, in which 
reaction consisteth the nature of sense, there will proceed an endeavor back to the retina. This endeavor outwards is the thing called light, or the phantasm of a lucid body. From this utter confusion of bodies, subjective and objective, physical and political, and motions, real and occult, proceed his deductions. It is no wonder that he had continual controversies about scientific questions, in which he was always worsted. One of the most famous resulted from his explanation of the barometer. In his desire to assume body for everything he was forced, as was Descartes, to include space in the category of substance. So to account for the space above the mercury, he asserted that air passed in and out through the mercury because he had previously stated that a vacuum was a body which could not be increased or diminished. Enough has been given to show that Hobbes was not a man of science and that his method was not the scientific method; and the same can be shown with certainty of all philosophers who flourished previously to the nineteenth century. It was not until then that a systematic and large accumulation of scientific observations of all sorts was at hand. Even now, the biological sciences are the only ones possible as a guide to ethics because the mathematical sciences, physics, astronomy, and chemistry, are too remote from human passions and emotions to be serviceable.

Apparently in the biological sciences it was neces- 
sary to wait for the promulgation of the law of evolution before a nexus could be formed between the material and spiritual aspects of nature. For it seems clear that such a system of ethics must substitute the worship of nature for the worship of God if science is to become the guide to conduct. That is, some principle of nature, subject only to physical laws, must work toward perfection in man in the ordinary sense of guiding his aspirations toward absolute goodness, and thus supply a check and interference to his actions; a check which metaphysical systems have always accomplished by assuming an outside and supernatural force, which is not restricted by natural law.

On first sight, the biological sciences, with evolution as a guiding principle, offer a promising field for a system of ethics which shall depend on natural law and be positive in that it does not introduce the occult and supernatural. It seemed necessary, during the Victorian age, only to have implicit confidence in evolution and to let the machine work out its own destiny, confident that all was well. Thus we have doctrines of humanitarianism and evolution in the poetry of Tennyson who trusts to that "one far-off divine event, to which the whole creation moves." We have the same confidence shown by Wordsworth who sucks his morality from flowers and stones, or by Fiske who writes his moral confession in an essay entitled "Through Nature to God." But all is not so simple or even so 
scientific as Spencerian philosophy would have us believe.

The doctrine of evolution, as a strictly scientific law, states merely that the different species of animals and plants were not created as distinct types but that they have gradually changed by a progressive modification until each existing species is the last surviving branch or twig of the biological tree. Evidently, then, all life can be traced back, if we could gather up the tangled and broken skein of life, either to one or at least to a few prototypes. Now the law of evolution takes no account of good or bad, of high or low, or of the method by which one species has changed to another. According to it, each type has maintained its continued existence simply because certain of its attributes have given it an advantage over its competitors. From observation we find that those types, we think to be primitive, are simpler in construction and functions than are those we suppose to be later. But it is by no means a universal law that simple types change to complex ones, witness the persistence of microbes and bacilli. The law of evolution is thus a scientific law which attempts to generalize a set of phenomena observed objectively and has nothing to do with ethics or what I have called character.

What may be called the ethical aspect of evolution has been injected into it by hypothetical or metaphysical reasoning. Darwin and his successors, generally, have 
attempted also to find the causes of evolution and the mechanism of heredity. And here all is confusion, and, as occurred in the physical sciences, each theorist sets up his own scheme of natural selection, of pangenesis, of mutation and what not, and the battle without an issue rages fiercely. In the first place, a purely anthropomorphic aspect is given to evolution by the unjustifiable use of the terms higher and lower forms of life. These words can signify merely the comparison between two types as regards their complexity of functions. It is only by regarding man as the goal toward which all creation tends, that we can call those types the highest which most nearly resemble man. The final step is easy and the word " high" in evolution is transferred to the derived sense of meaning noble and good. It would be a most interesting and useful work to trace back this curious and persistent habit of linking high and low as space attributes, to high and low as ethical concepts. Much of our confusion of thought comes from such loose use of words, and few of us can quite rid our minds of the impression that hell and evil are beneath us, and heaven and joy above us. At all events, the metaphorical use of these words seems to have done much to change the impersonal law of evolution into a sort of deity which develops the lowly algæ into a choir of angels.

If we attempt to discover, in a general way, the cause of evolution, we must ascribe it either to a super- 


\section{THE LIMITATIONS OF SCIENCE}

natural or to a natural force. The variation of species may be due to a supernatural power which created a protoplasm and then guided organic life through the slow developing ages. But to us such a form of creation is as little scientific as the cosmogony of the Book of Genesis. Scientific hypotheses must avoid the supernatural and, however they differ in details, they must base evolution on the natural laws of probability and chance, using these words in their technical sense of expressing the statistical actions of physical, chemical, or biological forces on molecular masses. In natural or scientific law there can be assumed no escape from the calculable action of a force. Given effects follow from given causes and these must precede each other with uniform regularity, back as far as we care to carry the law. In this manner we have formulated a law of evolution without introducing the idea of ethics at all. And so far as the question concerns ethics it is restricted to a minute province, man, in the vast empire of life. Thus we have made an absolute break in the law of evolution; on one side is the unmoral development of all the universe, and on the other side is the moral growth of man. At least in our egotism we reserve the attribute of character, or of judging actions as good or evil, to ourselves. It is almost useless to add that a law of continuous development, like evolution, with such a break in it is far from perfect. 
We shall therefore confine ourselves to the law of evolution only so far as it relates to man. And let us in the first place reject rigorously the sentimental opinions of a class of writers who twist the laws of nature into a kind of beneficial providence. The inexorable forces of nature teach us the survival of the strong and the elimination of the weak; the fatal progress of the inanimate universe riding over and crushing whatever disobeys its laws, without hate but without ruth, is not calculated to inspire an enlightened and exclusive student of science to regard with consideration this personification of nature as a type of human love which would cherish the sick and teach self-abnegation to the strong.

Nor can we discover, during historic times, much change in the physique or in the character of men. And we are forced to agree with Huxley, who believed that the laws of natural evolution ceased to be operative on man when self-consciousness became developed in him. In the prolegomena to Evolution and Ethics he introduces a vivid picture in which he portrays natural evolution of animals and plants as life in a forest. The characteristic feature of this life is an intense and unceasing competition in the struggle for existence. In the untamed forest, the cosmic forces use unrestricted multiplication as their principal aid; for every violet or for every oak that grows, thousands die, crushed without a compunction. There must be 
strength and flexibility and good luck that plants and animals may attain congenial places and invade successfully large areas. On the other hand, the habitation of man may be considered as life in a cultivated garden, where the gardener restricts multiplication, gives each plant space and nourishment, and shields it from the vicissitudes of climate. He uproots the natural inhabitants, modifies the soil and conditions to suit exotics, and gives the weak equal advantages with the strong. As a result, natural evolution has become so modified and weakened that man is in a class by himself, and develops under different laws from the rest of the world.

So far as I can see, nothing in biology has been discovered which contradicts this opinion of Huxley. It is, however, difficult to see how and when a break of so fundamental a nature between man and the rest of life could occur in a continuous evolution in which man is included. But it seems certain that man has attained to so complex and rich a character, his aims are so diversified, that any law of evolution of his future development simple enough for us to comprehend is out of the question. Where any such a law would operate to emphasize a certain attribute, it would diminish another which would be considered by many to be equally important. Instead of strength, flexibility, and good-luck only, there are a thousand other influences to be balanced. 
If we are to found ethics on biology and evolution we are driven to inventing a sort of spiritual evolution which is best expressed by the term naturalism. And if anyone will consider the systems of this kind which are proposed as guides to society, I think he will find that they may all be included in this one general type. There seem to be four possible ways of accounting for an evolution of the race. Humanity may be guided to a goal of perfection by some divine and omniscient external power which imposed certain initial laws of nature and permits a subsequent evolution to progress without interference; humanity may contain in itself such a directing force; it may be constrained toward a future condition by the conscious effort of a set of judges who will determine and enforce the manner of its evolution; lastly, society may divide into two classes, those who by superior knowledge and power fulfill their destiny at the expense of the opposite class of the ignorant and weak.

If we bear in mind that science is not only the study of natural law as an objective series of events, but that, if it is to have any application to the needs and desires of society, the natural balance and operation of forces and energy must be interfered with, then it is easy to see that the first two doctrines of naturalism are not scientific. To say that the human race is guided to a goal of perfection, previously determined, by either an external and divine power or by some 
omniscient power existing in society itself, is to deny and abstract from the individual the basic axiom of science that the laws of nature may be interfered with.

The third class, which leaves the guidance of the evolution of the race more or less under the control of individuals composing it, is properly a scientific method, because it postulates an objective world subject to laws and permits of their modification. This type of naturalism has already, under the name of eugenics, attained a very considerable vogue, and it is heralded as the long sought guide to righteousness. It is worth while to consider its claims both as a science and as a system of ethics.

Since the science of physics has developed the scientific method more consciously and more accurately than any other of the sciences, we may turn to it for an illustration of the method to be followed by eugenists. In the first place, the experimenter puts himself in the position of being external to the phenomena he expects to investigate. He also decides beforehand on the object of the experiment; that is, he has some definite idea which he wishes to confirm or contradict. He then observes and tabulates the phenomena which are relative to the problem and abstracts from them all the actions which complicate the result and are not essential to it. But as he invariably finds that the free and unconstrained phenomena are too com- 
plex to be manageable he must interfere with natural actions and rearrange them. Lastly, his observations are to be classified by a logical process into laws.

To apply this scientific method to the development of an ethical system which shall conform to the conclusions of biology, certain individuals or a class of individuals must act as experimenters, distinguish themselves from the rest of society, and regard its actions as objective phenomena which do not apply, for the time being, to themselves. These experimentalists must acquaint themselves with the object of their experiment and interfere with the unconstrained actions of society by providing constraints which shall control and direct its complex course into simpler channels; and finally they must coördinate the effects of these constraints.

Such should be the aim of what is popularly known as the science of eugenics. By derivation, eugenics means to be born well, and since the results of good birth may be counteracted by the subsequent life of the individual, a second science has been added currently under the title, euthenics, which may be defined as the science of living well. For the sake of simplicity let us use the word, eugenics, to include this entire system of scientific ethics. And let us put out of our minds immediately the idea that the eugenists are concerned with the simpler problem of the well-being of the individual; like all avowed men of science, they attempt 
to deal with classes of phenomena. In this instance, the object is to mold the whole human race, with its immensely complex and diversified desires and actions, its egotisms and its sympathies, into a homogeneous society which shall progress toward a standard, previously determined, of a noble and god-like humanity. The reward to be expected by the individual who is born and lives well is that intense feeling of satisfaction he will have that the race as a whole has been carried a little closer to a distant and vague goal of perfection because of his submission to the laws of eugenical righteousness.

We can now define the eugenists, if our ideas be correct, as a band of human beings who shall be the arbiters of right and wrong. This band must be small because it is difficult to find even two persons who have the same standards; it must be select as there is at present no known way for a person to exercise a choice in his parents and it will need judges of superior ability to pick out those who are worthy to be parents and to live. Parentage under eugenical guidance is certain to be a very serious matter and I imagine that we should expect a rather large diminution in the human race for a few centuries until the system is running smoothly. At present, we cannot leave the regulation of life after birth according to euthenic standards to the ordinary person, because we should then not have euthenics at all, but the present hap- 
hazard society. So we must take it for certain that all people must be born and live according to the regulations of a band of superior human beings.

Having thus settled on the organizers of experiments for this science, their next step will be to arrange the experiments and to carry them out. They will first, in order to work intelligently, decide what are the qualities of human perfection. I cannot discover that this has been done. There seems to be just as great diversity of opinion as to what constitutes the perfect man among the eugenists as there is among ordinary men. We meet so-called eugenic babies and euthenic adults, but as standards they are rather disappointing and do not seem to be sufficiently differentiated from other people. Perhaps this is to be expected, for true eugenics is a slow process. With the goal established, the experimenters must then find some scientific way of propagating the race eugenically and of determining and applying constraints or checks on our free manner of life which will make all individuals live euthenically.

If such a scientific method were practicable, it would seem to a physicist that a system of ethics could be established by it. But it is a question whether such a system, minimizing as it must the check of personal responsibility, would not fail because it would result in a form of intellectual slavery. So far the doctrines of modern eugenics have not shown the slightest indica- 
tion of scientific method; they are vague and characterized by a lack of any accurate thinking. Indeed, it is difficult to obtain an idea of what the eugenists aim to do or how they expect to work. Curiously enough, considering his lack of scientific training, Plato seems to be the one who saw the problem clearly and attempted a real solution. His belief, that every person desired to be born well and to live well if only he knew how, appeals to one as an explicit statement of eugenics. That is, if we could eliminate ignorance and regulate our passions, society would advance to an ideal state, and in his Republic, Plato sketches such a polity in detail. The failure, for failure it was, came from his inability to define what ignorance is or how to check our passions. His ideal state is not only impracticable, but if adopted would result in political slavery.

If we examine somewhat in detail what is being done to make a science of eugenics and to apply it to regulate the affairs of men, we shall find that it is in no sense a science and is singularly unfit to accomplish such a purpose.

In the first place there are no judges who are accepted, or likely to be accepted, as having any unanimity of purpose or plan. Ethical systems, in the past, have been born in the heart and mind of a single man, who was recognized as one endowed with a peculiar power of righteousness and whose life had become an ex- 
ample. So necessary was it to have an example of perfection which should not be subject to the waywardness of human passions, that without exception all religions assign divine attributes to their founders. And in order to maintain the integrity and the unanimity of belief in even a sect, the rest of society, with its conflicting purposes, has always been explicitly banished from communion with the faithful.

The case of eugenics is quite different. To be a science it must deal with the race and not with individuals; instead of a founder, to whom are ascribed divine or at least superhuman powers and who makes an appeal to sympathy, it must rely on a body of judges who appeal to law and reason, and who have discarded the powerful check of personal responsibility and personal reward. Instead of choosing individuals from society to form a more or less cohesive sect, the eugenists must constrain all individuals to forsake their personal desires and attempt to bind these heterogeneous units into a homogeneous race. And who are intellectually wise enough to be the arbiters of fate? The clinging of man to the idea of corporal manifestations of divinity is a confession of the impotence of man to grasp the problem of humanity. Huxley stated this difficulty unanswerably long before eugenics came to life. He, the evolutionist and biologist, warns us: "I doubt whether even the keenest judge of character, if he had before him a hundred boys and girls under 
fourteen, could pick out, with the least chance of success, those who should be kept, as certain to be serviceable members of the polity, and those who should be chloroformed, as equally sure to be stupid, idle, or vicious. The 'points' of a good or bad citizen are really far harder to discern than those of a puppy or a short-horn calf; many do not show themselves before the practical difficulties of life stimulate manhood to full assertion. The evil stock, if it be one, has had time to multiply, and selection is nullified."

Instead of a sober and careful study, with a full realization of the almost hopeless difficulties of the problem, we have a number of people, mostly of a very restricted outlook, who say with conviction that man is mentally defective, man is diseased, man is criminal. And they say vaguely, these misfortunes must be stopped. But how is any one of these to be remedied or how are we to weigh or balance them? A man may be a criminal and otherwise a perfect physical creature; a man may be diseased and yet be intellectually and morally a giant; in fact, Lombroso claims that all those we most reverence for morality or intellect were diseased and quite unfit to be progenitors of the race. And while this doctrine of Lombroso, that intellectual and moral genius is a symptom of physical degeneracy, is a monstrous falsehood, yet it is certain that those who possess great powers themselves frequently do not transmit their excellent qualities. And these are only 
the simplest attributes of a good citizen to be considered.

So far eugenics has limited itself to a trite formula that only the fit should be permitted to have children. This battle-cry has been sung loudly and it has gathered together a motley band who would interfere with the laws of nature and reform civilization overnight. They have no clear idea who are the fit or how the unfit are to be restrained. They busy themselves collecting statistics, but for the most part these are undigested, or are drawn from questionnaires scattered broadcast and inviting inaccuracy, and frequently they are not even honest. Books also are written and as an exposition of an ethical system they give the impression that the writers' loftiest aim is to turn the human race into a stock-breeding farm. The chief argument and the complaint is that men can breed cattle, dogs, and plants eugenically but not themselves. But what an absurd attitude this is! The breeder of dogs and cattle stands in a relation to them much as a god would to us. Dogs and cattle are bred to bring into prominence some trait which will suit the purpose or pleasure of their master, not of their own. No one supposes that the monstrous nose of the pug dog or the inflamed liver of the Strassburg goose was developed with any regard to those unfortunate animals. The whole point has been missed. Man cannot be bred like animals because he has no apparent master. If we could be 
content to believe that the destiny of man is under divine control, we can have hope; and if it is left to the rough methods of nature, which sooner or later blot out the degenerate and the weakling, there is some possibility of achievement.

In spite of much noise about the science of eugenics, the eugenists know that at most it is but a police regulation. Society has always assumed the right to protect itself by isolating or by punishing, even with death, those it considers dangerous to its stability. The eugenists are advocating the same thing and have so far limited their efforts to prevent children from criminals, imbeciles, drunkards, and syphilitics. The methods of society to accomplish this safeguard have been crude, but they have been sufficiently effective to prevent any notable deterioration of the race, and it is safe to say there has been improvement. If the eugenists would try to improve the methods of the past to some extent, something might be done. But there is reason for believing that even this good is likely to be stifled by the outcries and rash heedlessness of the overzealous.

To prevent the strains of disease, imbecility, and criminality from continuing in the race, we now have the proposed surgical remedy of vasectomy. As an efficient preventative it must be a failure. No society would countenance such an operation except for cases of hopeless degeneracy. While it might reduce somewhat the number of degenerate offspring, we would 
still have the children of the less degenerate, who may inherit the weakness of the parent in an intensified form. No good arguments can be given against the more humane method of the separation of sexes in institutions. This method requires merely a reform in such institutions as already exist in order that their inmates may be provided with as free and useful life as is possible. The argument is frequently given that the cost of this method is great. But a strong and vigorous community may well blush at such a motive; certainly the care of its unfortunates is a solemn duty and it should be undertaken with as much solicitude as a man would shield a weakling child. And as for the plea that, after this operation, the individual loses only the power and not the desire to procreate, it strikes one as simply disgusting. Is it not giving a license to the degenerate and to the man of loose morals to be as foul as he pleases if only his sins end with himself? It would be far more manly, if the plan of isolation cannot succeed, to put degenerate children and adults to death and at least preserve some self-respect for the dignity of human life.

Another plan proposed to promote eugenics is to rely on the legislator and the clergy to prohibit marriage, unless the contracting parties show certificates of fitness from a physician. Without the least knowledge of what effect such a regulation would have, the state of Wisconsin has recently passed a law of this 
character. The result so far has been ridicule and a marked decrease in marriages. The absolute inability to carry out the purpose of such a law does not seem to have occurred to its promoters. If it were enforced over a wide area, the youth would certainly dispense with the ceremony of marriage rather than to debase their ideals of love. And one can imagine the consternation of physicians if they were forced to decide when people were fit to marry. Very few of them know anything about the laws of heredity, and those that do know the laws of heredity also know that they are so complex and so obscure that only a few extreme cases can be trusted. As Huxley said, the points of a good citizen are more difficult than those of a puppy or a short-horn calf.

A third class of eugenists consists mostly of the hysterical element of the social workers who sob over the sins of society and sob over the innate purity of the harlot, who weep over the heartlessness of the lawabiding and weep over the innate nobility of the criminal. So far as one can make out from their incoherent utterances, they wish to put all the sins of the individual on society, without comprehending that society is a collection of individuals. Whatever good they may accomplish, no one in the least conversant with science will concede that they are advancing an ethics in conformity with scientific methods; for if science makes any one thing clear, it is that the actions of the indi- 
vidual must bring their reactions also on the same individual.

Now it seems to me that in eugenics of the simplest type there is a possible good. If the advocates of good breeding, and everyone is that to some extent, will work quietly and systematically on the problems of heredity; if they will try to find the effects of criminality and of disease on posterity, we shall then have some reliable facts to work on. We may then hope to accomplish some more decent plan of isolating the worst of such cases than we have now. And we are sure of the help of society itself, for we know that the human race contains in itself the power of gradually eradicating defective stock from the simple fact, that if it did not possess this power, it would have hopelessly degenerated long ago. But this natural process is slow and if it can be accelerated and guided, eugenics will be valuable.

There are many of these patient and accurate investigators of the laws of heredity but there are also unfortunately others, classed professionally as eugenists, whose methods are not so reliable. From their bureaus issue questionnaires which are spread broadcast. The statistics which result are mostly useless and often false, as they are given by untrained people. And they are especially obnoxious as they too frequently make a direct appeal to the morbid. Their so-called laws are based on insufficient evidence. Apparently 


\section{THE LIMITATIONS OF SCIENCE}

the only two examples which have been worked out in America are the Jukes and the Edwards families. The first has shown itself to be an undesirable and the other a desirable strain. But curiously enough the first ancestress in America of each of these families was not a moral woman. It is sufficiently easy to look back and decide what characteristics proved to be undesirable, but it is a very different thing to look forward and determine who should establish a family and who should not. Not even the most enthusiastic eugenists can hope to unravel the genealogies of more than a small proportion of families. And it would require accurate genealogies of many families, not only of successive generations but also of the characteristics of its members, to settle whether a strain of immorality came simply from an excess of vitality or was the result of degeneracy.

If eugenics is a problem too complex for solution, its companion, euthenics, is an example of trying to assign to science a problem it has no means of solving. Science knows no method of constraining an individual to conduct himself so as to further the existence of a perfect race. The greatest difficulty in all ethical systems is to provide an efficient check on the passions. The strongest check is evidently the belief that disaster to himself will result from disobedience of the laws of right conduct. And yet the man is rare who can by his will refrain from those habits and 
passions which he knows to be injurious to himself or to those nearest and dearest to him. What more ineffective system of ethics could be imagined than euthenics, which bases its claim on the plea that a man should so live that the race may move forward to some unknown goal of perfection? And he is not even to have the satisfaction of seeing the progress of the race, as little improvement is to be expected for a hundred or so of generations.

The average man is too busy with his own concerns even to consider those of a distant posterity, of whose needs he can know nothing. $\mathrm{He}$ is convinced that future generations cannot be controlled by him and that they must solve their own problems; and he is also conscious that he is not altogether degenerate although he is the result of a long line of careless ancestors, heedless of him and ignorant of eugenics and euthenics. However traits of heredity may affect immediate posterity, it must be recognized as a general principle that the race has attained a normal development, which can change but slightly and very slowly; disease and vice must disappear just as abnormal excellences must decay. We find but little that is essentially different in the moral character of ourselves and that of persons who formed the ancient civilizations. The eugenists forget that the great majority of all men, and a still greater majority of those of influence, lead for the most part decent, law-abiding lives, and this quiet 
and unobtrusive power of example is far more potent for righteousness than a propaganda led by enthusiasts.

Side by side with the doctrine that human sympathy is the controlling factor of ethics, and this belief is evidently the basis of eugenics, there has always persisted the contrasted doctrine that the state of man is one of warfare, a survival of the fit. This school evidently relies on a law of natural evolution based on the motive of egotism. It is thus my fourth class of naturalism.

Of all those who have advanced this motive of egotism, none has done so as explicitly, or has made of it so complete a philosophy as Hobbes. "In the first place," he says, "I put forth, for a general inclination of all mankind, a perpetual and restless desire of power after power, that ceaseth only in death." This is, I think, the direct influence of science unqualified by character and piety. It is not my purpose to follow this doctrine down to the present time but it can be shown that Nietzsche, with his ideal of the Superman, is the logical successor of Hobbes. Both the strength and weakness of this form of philosophy have been contrasted in a recent essay: *

"Nietzsche regarded the self-assertive Superman as a true reaction against the prevalent man of sympathy, and as a cure for the disease of the age. That

*Essay on Nietzsche. By Paul Elmer More. Shelburne Essays; Eighth Series. 
much of Nietzsche's protest against the excesses of humanitarianism was sound and well directed, I for one am quite ready to admit. He saw, as few other men of our day have seen, the danger that threatens true progress in any system of education and government which makes the advantage of the ordinary rather than the distinguished man its first object. He saw with terrible clearness that much of our most admired art is not art at all in the higher sense of the word, but an appeal to morbid sentimentality. . . . But the cure Nietzsche proposed for these evils was itself a part of the malady. The Superman, in other words, is a product of the same naturalism which produced the disease it would counteract; it is the last and most violent expression of the egotism, or self-interest, which Hume and all his followers balanced with sympathy, as the two springs of human action."

If the predominant object of science is to acquire power, how can we escape the conclusion that if it should become the arbiter of ethics, society would tend to a condition closer to the ideals of Nietzsche than of sentimental eugenics? Can we look with complaisance on the unrestricted development of either of these ideals? Nietzsche teaches a gospel of scientific evolution when the restraints are removed from the free exercise of self-interest. The gospel of the Superman, when transferred to the ambitions of a nation, as has been done by Treitschke, shows its results in that doc- 
trine of necessity of the Germans which has plunged Europe into war. No individual and no nation can believe for long that in him or in it rest the culture and the power of the world without resorting ultimately to the arbitrament of force to overcome opposition. And on the other hand, the ideal of a world governed by human evolution and depending on brotherly love - a world under a banner floating from a Peace Palace of The Hague-is a dream of sentimentality. And yet, one or the other seems to me the goal of eugenical righteousness. But because science is not concerned primarily with problems of character, it does not follow that its pursuit does not promote character. We certainly enrich the character by the larger acquisition of power which results from the modifying and dominating of our environment. This is true, if side by side with increased power to do things, we also learn to use it for the benefit of humanity; that is, when it is regulated according to the inner check of piety, as taught by those in whom we recognize the mysterious power of righteousness.

If I have made myself clear, the limitations of science are due solely to the fact that there are, in addition to material forces, others of an essentially different kind which may be called, for lack of a better name, spiritual powers. And so long as men of science restrict their endeavor to the world of material substance and material force, they will find that their 
field is practically without limits, so vast and so numerous are the problems to be solved. And it should distress no one to discover that there are other fields of knowledge in which science is not concerned; on the contrary, the fact that the range of science is limited should encourage us to greater hopes, because our freedom of action is still far greater than our powers of accomplishment. After centuries of effort, the ocean of the unknown lies before us unexplored. 



\section{INDEX}

Abstractive and hypothetical methods, I3; outlined by Rankine, 20.

Action at a distance, 84, 134 .

Ampère, abstractive method, I90.

Aristotle, continuity, 77 ; dictator of Greek and medieval science, 230; spirit of Greek science, 23I; ideas as logic, $23 \mathrm{I}$.

Astronomy, its relation to hypothesis, 76 .

Atomic, Kelvin's models, 36, 190; nature of electricity, I38.

Atomic theory, defined, 8; its axioms, II; failure, 24; not an aid to discovery, 33 ; inadequacy of, 44 ; modern and classic, 49; its nature, 73 ; its philosophical weakness, II9; its value, I3O.

Atoms, nature of, Io; Larmor's definition, 26,49 ; necessary attributes, 44 ; subdivided, 44; modern conceptions, 129.

Axioms, of atomic theories, II ; see Postulates.

Bacon, Fr., natural philosophy, I2I; province of mathematics, I42; on the sources of knowledge, 233 ; on the extent and limit of science, 234.

Biology, as the basis of ethics, 236.

Bolingbroke, Lord, critique of Descartes's cosmogony, I04, I44.

Boscovich, atomic theory, 8.

Bradley, discovery of the aberration of light, 19.
Browne, Sir Thomas, nature of electricity, 108.

Campbell, Norman, division of past and present science, 39.

Celestial matter, nature of, 90 .

Character, not the aim of science, 229.

Clifford, W. K., plurality of ethers, II.

Cohesion, cause of, Descartes, 92 ; Larmor, 127.

Continuity and discontinuity of matter, contrasted, 3, II9; as a dualistic principle, 40, 44 ; ideas on, 77 ; its history, 77 ; conflict between, 78 ; inability to decide between, 143.

Cosmogony, of Laplace and Lagrange, 7; Larmor's, 47; requirements for a scientific, 72 ; nebular hypothesis, 74 ; as a scientific convenience, 80 ; Descartes's, 82 et seq.; revealed and natural, 240 .

Coulomb, nature of electricity, 153.

d'Alembert, laws of motion, 6 . Dalton, atomic theory, 33 .

Darwin, law of evolution, 226, 238.

Democritus, atomic theory, 8 , 73.

Descartes, influence on science, $3,5,39,40,67$, I0I, 142, 224 ; theory of matter, 40; the plenum, 40, 78; example of hypothetical method, 70 ; exponent of continuity of matter, 78; scientific principles, 79 ; revelation, 80 ; attributes of substance, 82 ; action at a distance, 84 ; laws of mo- 
tion and impact, 85 ; classification of matter, 86 ; vortices, 89; cohesion, 92 ; light, 92; heat, 95; the earth, 96; his consistency of thought, 98 ; critique of his cosmogon y, IOI, Bolingbroke's opinion, 104; nature of electricity, 107; space as substance, 236.

Dufay, fluid theory of electricity, 25.

Duhem, on science of energetics, 22; on theories of physics, 37 .

Egoism, as a scientific system of ethics, 258.

Einstein, definition of space, 109, I27, I53; electricity and energy as entities, III.

Einstein's Principle of Relativity, to harmonize modern and Newtonian mechanics, 164 ; its postulates, 165 ; measure of time, I67; measure of length, I69; variability of mass, 172; mass and energy, I72 ; its postulates, I74; restriction of the relativity of motion, 175; absolute velocity of light, 177; velocity of sound and light, an illustration, 182 ; atomic nature of electricity, 184; logical but false, 184 .

Electricity, an entity, 42, 51, 63,65 , 107, I10, 184; the basis of mechanics, I 58 .

Electrons, nature of, 55,60 , I31, I35, 138.

Energetics, definition, II, 20 ; value of, 24 .

Energy, the basis of science, 22 ; conservation of, 120,128 ; classification, 122, I33.

Ether, a mechanical link, ro; need for more than one, II; and matter, 42 ; its properties, 43; elastic-solid and electromagnetic, 52 ; massive, I09; recent ideas of, 109; a confession of ignorance, 125 ; a plenum, 126; a new conception of the, 162 ; a convenience only, 198; as a reality, 202; as the explanation of matter, 203; the reductio ad absurdum, 204; Lodge's definition, 208; as a psychic medium, 208; as a quasi-deity, 209.

Ethereal, see Vortices.

Ethics, present perplexity, 213; science as the basis of, 214 ; lack of criticism of scientists, 215; effect of hypothesis on, 225; materialism, 233; atheism, 233 ; system of Hobbes, 235 ; science, the guide to conduct, 237 ; law of evolution not ethical, 238; evolution and, $24 \mathrm{I}$; classification of naturalism, 243; egoism, 258; relation of science to, 260 .

Eugenics, as a science, 245; as a system of ethics, 249; as a police regulation, 252; under guidance of physicians, 252; under guidance of legislators and the clergy, 253; under the social workers, 254; lack of influence, 257.

Eugenist, defined, 246.

Euthenics, defined, 245; its failure, 256.

Evolution, as a law, II6; social, 212; discussed, 237; its causes, 239; natural and restricted, 24I ; spiritual, four types of, 243.

Experience, the basis of science, 233.

Experimentum crucis, 18.

Fallacies, in Descartes's system, 98.

Faraday, the duty of science, 107; theory of electricity, 108; effect of dielectrics, 160; nature of the ether, I6I. 
Fire, Descartes's conception of, 90.

Fiske, John, naturalism, 237.

FitzGerald-Lorentz, effect of motion on matter, 160 .

Fizeau, the velocity of light in moving media, I77.

Force, as the basis of science, II 8.

Foucault, velocity of light in transparent media, 19.

Franklin, Benj., fluid theory of electricity, 25 .

Galileo, on sensation as the postulate of science, 32 ; founder of experimental science, 39,187 ; classical mechanics, I42.

Gassendi, atomic theory, 8, 85 .

Genealogies, eugenics, 255.

Generalization, a necessity, 194.

Geometry, its postulates compared with those of experimental science, 12; the basis of science, 79 .

Goethe, restraint in science, 58.

Guericke, von, theory of electricity, 108.

Hannequin, atomic theory, I ; definition of mechanics, II8.

Heat, Descartes's hypothesis, 95.

Helmholtz, von, conservation of energy, 86; vortex rings, I3I ; stagnant ether, I6I.

Heraclitus, continuity of matter, 77.

Hertz, H., opinion of Maxwell's equations, 37 ; electromagnetic waves, I60.

Histories of science, their deficiencies, 217.

Hobbes, on the sensations, 233 , 234; metaphysical system, 235 ; on the barometer, 236 ; egotism of mankind, 258.

Hume, on the sensations, 233; egotism and sympathy, 259.

Huxley, on evolution, 24I; on eugenical selection, 249.
Huygens, atomic theory, 8, 40; theory of light, I9; correction of laws of impact and motion, 86.

Hypothesis, its danger, I6; elimination not desirable, 37 ; utility of, 42 ; apology for, 57 ; not an indifferent subject, 59; Descartes's, the most complete, 98 ; good and bad aspects, I03; Bolingbroke on, I04; and symbolism, IIO; a tentative hypothesis of electricity, 140; its value, I44; effect on society, 192; as generalization, 195; Poincaré's classification, 200; and ethics, 225.

Hypothetical method, I3; its deceptions, 70.

Idolatry of scientists, 209.

Impact, Descartes's laws, 85 .

Johnson, Samuel, reality of matter, 206.

Kant, axioms of mechanics, 6 ; nebular hypothesis, 74 ; space and time, I48.

Kaufmann, variation of mass with velocity, I32, I37.

Kelvin, Lord (Sir Wm. Thomson), models of atoms, 36 , 190 ; theory of vortex atoms, 4I, 48, I3I ; size of atoms, 44; origin of life, II7; quantitative measurements, 145.

Lagrange, axioms of mechanics, 6 ; equilibrium of bodies, 6; Mécanique analytique, 7 ; no criterion for truth, I8.

Laplace, Système du monde, 7 ; materialistic attitude, 8, 212; his mechanistic theory, 9; nebular hypothesis, 74 ; velocity of propagation of gravitational energy, II 8 ; on the inductive method, 232.

Larmor, Sir Joseph, properties of the ether, 19, 53, 126; 
corpuscular theory of matter, $26,27,4 \mathrm{I}, 42,44, \mathrm{IIO}$; value of speculative hypothesis, 43 ; continuity of matter, 46; his hypothesis does not avoid former difficulties, 50; divisibility of matter, II9; definition of ether, I26; electrical theory of mechanics, 127; definition of the atom, 129.

Laws, difference between hypothesis and, 104; continuity, causal, II5.

Leibnitz, invention of the calculus, 5 .

Length, a function of motion, I69.

Lewis, G. N., confusion of fact and fiction, II2.

Light, theories of, I9; Descartes's hypothesis, 92; its velocity, an absolute constant, 165; its velocity in vacuous and in occupied space, I77; its velocity not a maximum, 182 .

Limits of science, II3.

Locke, on the sensations, 233.

Lodge, Sir Oliver, the ether as a medium for psychic phenomena, 24; ether massive, IIO, I53; presidential address on Continuity, I93; his scientific credulity, I94; his scientific confusion, 207; his ideas of the ether as omnipotent and omniscient, 208; as a quasi-deity, 208; as a soul-body, 209; his speculations not logical, 209; war between science and theology, 2ro; as an example of scientific dogmatism, 2 IO.

Lombroso, the imperfection of the genius, 250 .

Lorentz, H. A., his corpuscular theory of matter, 26 , 28, 4I, 55, IIO, I30, I 44; properties of the ether, 56 ; apology for the hypothetical method, 57 ; ethereal stresses not realities, I09; contraction of matter, 160 ; ether stresses, I6I.

Lucretius, atomic theory, 73 .

Mach, Ernst, science of energetics, 22.

Mass, as an attribute of electricity, 64, II7, I35, I7I; as a fundamental unit, 145,148 , 153; Newton's conception, 155; as dependent on energy, 172.

Mathematics, as a scientific language, 71 ; its value, 150 ; a paradox, I5I; not subject to the restrictions of science, I85.

Matter, real and hypothetical, I7 : continuous or discontinuous, 40, 44; modern definitions, 50 ; identity of space and, 82 ; fundamental attribute of, 82 ; three primordial kinds, 88; terrestrial, 96 ; confusion with electricity, IIO; its divisibility, II9; true and sensible, 120; as Ding an sich, I48; our concept of, I50; as an ethereal strain, r6r ; Poincaré's definition, 205.

Maxwell, J. C., theory of light, I9, I60; theory of electrification, 25, 108; discredit of vortex theory, 48 , I3I ; origin of life, II7; nature of the ether, I6r.

Mechanical, rise of principles, 5 ; theories have no criterion of truth, 18; models do not lead to discoveries, 37 ; theories, their weakness, 62 .

Mechanics, dominance over science, 4; axioms, 6; its metaphysical basis, I2; the only complete theory, I3; its failure as a method, I46; Newtonian, 154; the new, I59.

Mechanistic idea of the universe, 8 . 
Metaphysical theory, Larmor's, 27 ; Lorentz's, 28; influence on thought, 28.

Metaphysics, definition, I.

Method, of physics a model, 3 ; abstractive and hypothetical, I3; Rankine's, 20; the author's outline, II5;

Laplace's opinion, 232.

Michelet, 69.

Michelson, A. A., ether drift, 159, I79; velocity of light in moving media, I77.

Minkowski, four dimensional space, III.

Models, for radiation, I23; see Atoms.

Moral influence of science, 213.

More, L. T., outline of a scientific method, II5; on ether stresses, I6r.

More, P. E., on Nietzsche and the doctrine of egoism, 258 .

Morley, Edward, on ether drift, I59, 179.

Motion, real and hypothetical, I52.

Naturalism, as social evolution, 243.

Nebular hypothesis, 74 .

Newton, on hypothesis, title page; calculus, 5 ; law of gravitation, 6, 2r, its application to atoms, $7,40,74$; hypothesis of light, I9, 2I, 226 ; action and reaction, II5; classical mechanics, 142, 154 et seq.; on mass, I55; replacing Kepler's laws, 203.

Nietzsche, Fr., reaction against humanitarianism, 258 .

Occult, substances and forces, 77.

Organon of Aristotle, the text for Greek and medieval science, 230.

Organum Novum of Bacon, the authority for modern science, 231.
Ostwald, W., science of energetics, 22.

Peripatetic school, 23r.

Phil os o phy, metaphysical teaching of scientific, I, 22I ; of Greeks characterized by Bacon, 23I ; of Hobbes, 234; scientific, 236 et seq.

Physical theory, nature of, 12; aims of, 37; originators of, $4 \mathrm{I}$.

Physics, has best scientific method, 2 ; relation to metaphysics, 3 ; dates from, 39.

Planck, Max, quantum theory of discontinuity, II9; on principle of relativity, 165 .

Plato, the world as idea, 231 ; and eugenics, 248.

Plenum, 40; Larmor's 46; Descartes's, 83.

Poincaré, H., on hypothesis of ether, 59; The Foundations of Science, 193; as a skeptic, 193; on the scientific method, I94; on generalizations, 194; on hypothesis as a convenience, 198; classification of hypotheses, 200; the new mechanics, 203; the function of science, 204; definition of matter, 205, 229.

Postulates of science, their truth, $8 \mathrm{r}$; extent, as a fundamental attribute, 82 ; of Descartes, 85, I48; of Einstein, 165; of the new mechanics, 174; their subjectivity, 219 .

Power, the aim of science, 214 ; as the desire of mankind, 258, 260.

Primordial. space and time, 72, 86.

Principia Naturae of Descartes, 79 et seq.

Principia of Newton, title page, I55.

Proclus, science as mathematics, 232. 
Radiant energy, nature of, 122; and its medium of transmission, i23, 128 .

Rankine, on energetics, II ; on physical theories, 12; outline of abstractive and hypothetical methods, I6, 21 .

Relativity, Principle of; see Einstein.

Relativity, of motion and position, 83; Descartes's doctrine, 83; of knowledge, 147; of mechanical motions, 175; not applicable to radiant energy, I75; an academic question, I82; limited to hypothetical cases, 184 .

Rémusat, C. de, Vie de Bacon, 233.

Renan, Ernest, science as symbol and law, 2, 212; social evolution, 212.

Roentgen, W. C., discovery of X-rays, 38.

Rutherford, Sir Ernest, experimental evidence for atoms, 6o; theory of radioactivity, 63 .

Science, as a guide, 2,58 ; its limits, 30 , II 3,228 ; catalogue of, $15 \mathrm{I}$; its functions, 187; its domination of modern thought, I88; its dogmatism, 189; lack of criticism, 189, 215; as a measure of civilization, 215; overthrow of Aristotelian, 230 ; its aim to acquire power and not character, 229; and ethics, a modern combination, 234; its ethical bearing, 259; its true aim, 260.

Scientific, method, I; cult, 35 ; and religious polemic, 35 ; skepticism and credulity, 193; dogmatism, 210; ethics, feasibility of, 2r4.

Scientists, effect of hypothesis on, 28; German school of, 206, 207; education of, 223.
Sensations, not criteria of phenomena, 8.

Sentiment, as the basis of hypothesis, 105 .

Space, and matter, 83; our concept of, I48.

Spencer, Herbert, evolution as a social law, 212, 238.

Superman, as the contrast to eugenics, 259.

Symbolism, the end of hypothesis, IIO; of the new mechanics, 206.

Tennyson, Lord, humanitarianism, 237.

Theology and science, 30, 35 , 58, 2 ro.

Theory, need at present time, 3.

Thomson, Sir J. J., properties of atom, I7; theory of matter, $41,54,129$; on electricity, I09; mass of electron, 136 ; artificiality of his hypothesis, 202.

Time, our idea of, I49; measurement of, 167 ; a property of space, r68; variability with motion, I7I; Einstein's idea of, $18 \mathrm{I}$.

Transcendental symbolism of German physicists, 206, 207. Treitschke, doctrine of necessity, 259.

Units, of measure, II8.

Universe as a machine, 9, 18 , 72,185 .

Velocity of light, its significance in the modern ether, I79.

Vortex, Kelvin's theory, 48; Descartes's theory, 87 .

Wilson, C. T. R., mass of the electron, 136 .

Wordsworth, William, humanitarianism, 237. 




\section{BERGSON'S CREATIVE EVOLUTION}

Translated from the French by Dr. Arther svitchell

8th printing, $\$ 2.50$ net, by mail $\$ 2.67$.

"Bergson's resources in the way of erudition are remarkable, and in the way of expression they are simply phenomenal. . . . If anything can make hard things easy to follow it is a style like Bergson's. It is a miracle and he a real magician. Open Bergson and new horizons open on every page you read. It tells of reality itself instead of reiterating what dusty-minded professors have written about what other previous professors have thought. Nothing in Bergson is shopworn or at second-hand."-William James.

"A distinctive and trenchant piece of dialectic. ... Than its entrance upon the field as a well-armed and militant philosophy there have been not many more memorable occurences in the history of ideas."-Nation.

"To bring out in an adequate manner the effect which Bergson's philsophy has on those who are attracted by it let us try to imagine what it would have been like to have lived when Kant produced his 'Critique of Pure Reason.'"Hibbert Journal.

"Creative Evolution is destined, I believe, to mark an epoch in the history of modern thought. The work has its root in modern physical science, but it blooms and bears fruit in the spirit to a degree quite unprecedented. . . . Bergson is a new star in the intellectual firmament of our day. $\mathrm{He}$ is a philosopher upon whom the spirits of both literature and science have descended. In his great work he touches the materialism of science to finer issues. Probably no other writer of our time has possessed in the same measure the three gifts, the literary, the scientific, and the philosophical. Bergson is a kind of chastened and spiritualized Herbert Spencer."-John Burroughs in the Atlantic Monthly.

H E N R HOLT A N D PUBLISHERS
C O M P A N Y NEW YORK 


\section{FIFTY YEARS OF DARWINISM}

Comprising the eleven addresses in honor of Charles Darwin delivered January, 1909, before the American Association for the Advancement of Science. $\$ 2.00$ net; by mail $\$ 2.16$.

Contents:- Introduction, T. C. Chamberlin; Fifty Years of Darwinism, E. B. Poulton; The Theory of Natural Selection from the Standpoint of Botany, J. M. Coulter; Isolation as a Factor in Organic Evolution, D. S. Jordan; The Cell in Relation to Heredity and Evolution, E. B. Wilson; The Direct Influence of Environment, D. T. MacDougal; The Behavior of Unit-Characters in Heredity, W. E. Castle; Mutation, C. B. Davenport; Adaptation, C. H. Eigenmann; Darwin and Paleontology, H. F. Osborn; Evolution and Psychology, G. Stanley Hall.

\section{KELLOGG'S DARWINISM TO-DAY}

By Vernon L. Kellogg, Professor in Stanford University. $\$ 2.00$ net; by mail $\$ 2.16$.

A simple and concise discussion for the educated layman of presentday scientific criticism of the Darwinian selection theories, together with concise accounts of the other more important proposed auxiliary and alternative theories of species-forming.

Its value cannot be over-estimated. A book the student must have at hand at all times, and it takes the place of a whole library. No other writer has attempted to gather together the scattered literature of this vast subject, and none has subjected this literature to such uniformly trenchant and uniformly kindly criticism. An investigator of the first rank, and master of a clear and forceful literary style.President D. S. Jordan in the Dial.

\section{LOCY'S BIOLOGY AND ITS MAKERS}

By William A. Locy, Professor in Northwestern University. $\$ 2.75$ net; by mail $\$ 2.88$.

An untechnical account of the rise and progress of biology; written around the lives of the great leaders, with bibliography and index. The 123 illustrations include portraits, many of them rare, of nearly all the founders of biology. The book is divided into two parts, Part I dealing with the sources of biological ideas except those of Organic Evolution, and Part II devoting itself wholly to Evolution.

It is entertainingly written, and better than any other existing single work in any language, gives the layman a clear idea of the scope and development of the broad science of biology.-Dial.

\section{HENRY HOLT AND COMPANY}




\section{BOOKS ON SOCIAL SCIENCE}

\section{SOCIALISM AND DEMOCRACY IN EUROPE}

By SAmuel P. Orth. With bibliography and "Programs" of the various Socialist parties. $\$ 1.50$ net.

Traces briefly the growth of the Socialist movement in France, Belgium, Germany, England, and attempts to determine the relation of economic and political Socialism to Democracy.

\section{MARXISM VERSUS SOCIALISM}

By V. G. Srmkнovitch, Professor of Political Science, Columbia University. $\$ 1.50$ net.

A thorough and intimate account of all the intricate theories, problems and difficulties of modern Socialism.

Professor Simkhovitch shows us that the economic tendencies of to-day are quite different from what Marx expected them to be and that Socialism from the standpoint of Marx's own theory is quite impossible.

\section{WHY WOMEN ARE SO}

By Mary Roberts Coolidge. $\$ 1.50$ net.

"A fearless discussion of the modern woman, her inheritance, her present and her more promising future. The eighteenth and nineteenth century woman is keenly analyzed and compared with the highest type of woman to-day." -A. L. A. Booklist.

\section{A MONTESSORI MOTHER}

By Dorothy Canfield Fisher. With Illustrations. $\$ 1.25$ net.

A simple untechnical account of the apparatus, the method of its application, and a clear statement of the principles underlying its use.

"Mrs. Fisher's book is the best we have seen on the subject."-Independent.

\section{THE SOCIALIST MOVEMENT (Home University Library)}

By J. Ramsay Macdonald, Chairman of the British Labor Party. 50 cents net.

Traces the development of Socialistic theory, practice, and party organization; with a summary of the progress of Socialist parties to date in the leading nations.

"Not only the latest authoritative exposition of Socialism, it is also he most moderate, restrained and winning presentation of the subject now before the public." - San Francisco Argonaut.

H E N R Y H O L T A N D C O M P A N Y PUBLISHERS 


\section{HEREDITY IN RELATION TO EUGENICS}

By Charles B. Davenport, Director, Department of Experimental Evolution, Carnegie Institution of Washington. With diagrams. 8vo. $\$ 2.00$ net.

Eugenics treats of inborn, inheritable capacities and tendencies. Modern heredity explains how these tendencies get into the children and assists young persons to select consorts so as to have a sound offspring. The book tells what is known of the inheritance of various diseases and other characteristics; it discusses the origin of feeblemindedness, its vast social consequences, and its elimination; it considers American families, the part they have played in history, and the proof they furnish of the all-importance of "blood."

"One of the foremost authorities ... tells ... just what scientific investigation has established and how far it is possible to control what the ancients accepted as inevitable. ... The scientific student of heredity will find in it the latest developments and achievements in his particular province. . . Nevertheless, it is meant more for the intelligent general reader, who will be able to read it ... with entire comprehension.... Many interesting instances that illustrate how good or bad tendencies, physical. mental, or moral, radiate from the focal point of a single individual or family." $-N$. $Y$. Times Review.

\section{THE EVOLUTION OF ANIMAL INTELLIGENCE}

By S. J. Holmes, Professor in the University of Wisconsin. With illustrations and charts. 8vo. \$2.75 net.

A general account of the evolution of animal behavior from the mollusk and crustacean up to apes and monkeys. The critical point of the transition from instinct to intelligence receives special treatment. One of America's leading scientific authors who saw the manuscript before publication writes, "Holmes's is the best of the lot, and on the whole the most interesting because it gives the most facts; i.e., examples, illustrations, incidents, stories, etc. Holmes is immensely well informed."

\section{PFUNGST'S CLEVER HANS}

By Oskar Pfungst. Translated by Cart L. Rahn, Fellow in Psychology in the University of Chicago. 12mo. \$1.50.

The performances of Clever Hans, the horse of Mr. von Osten, created throughout Germany intense excitement and a widespread, overheated discussion of animal reason.

"The book is a minute and careful examination of the facts in the case, and should be of interest to psychologists and to all students of the intelligence of the lower animals."-Springfield Republican.

\section{HEN R Y H C L T A N D C O M P A N Y PUBLISHERS




\section{WORKS IN PHILOSOPHY AND PSYCHOLOGY}

\section{Aikins's Principles of Logic.}

By Herbert Austin Aikins, Professor of Philosophy in Western

Reserve University. $\mathrm{x}+489 \mathrm{pp}$. $12 \mathrm{mo}$. \$1.50.

Angell's Psychology.

By James Rowland Angell, Professor of Pyschology in the Uni-

versity of Chicago. $12 \mathrm{mo}$. \$1.60.

Baldwin's Elements of Psychology.

By James Mark Baldwin, Professor in Johns Hopkins University.

$\mathrm{xv}+372 \mathrm{pp} . \quad 12 \mathrm{mo} . \quad \$ 1.60$.

Baldwin's Handbook of Psychology.

By James Mark Baldwin, Professor in the Johns Hopkins University.

Senses and lntellect. xiv +343 pp. 8vo. Revised Edition. $\$ 2.00$.

Feeling aNd Will. xii +394 pp. 8 vo. \$2.00.

Colegrove's Memory.

An Inductive Study. By F. W. Colegrove. With an Introduc-

tion by G. Stanley Hall, LL.D. $369 \mathrm{pp} .12 \mathrm{mo}$. $\$ 1.50$.

Dewey \& Tufts's Ethics.

By John Dewey, Professor in Columbia University, and James H.

Tufts, Professor in the University of Chicago. (American Science

Series.) $618 \mathrm{pp}$. 12mo. \$2.00.

Falckenberg's History of Modern Philosophy.

Nicolas of Cusa to the Present Time. By Richard Falckenberg,

Professor in the University of Erlangen. Translated, with the author's co-operation, by A. C. Armstrong, Jr., Professor of Philosophy in Wesleyan University. xvi +655 pp. 8vo. \$3.50.

Hyde's Practical Ethics.

By William De Witt Hyde, President of Bowdoin College. xi +208

pp. $16 \mathrm{mo}$. \$1.00.

James's Principles of Psychology. Advanced Course.

By William James, Professor of Psychology in Harvard University.

Two volumes. 8vo. (American Science Series.) \$5.00.

James's Psychology. Briefer Course.

By William James, Professor in Harvard University. xiii $+478 \mathrm{pp}$.

I2mo. (American Science Series.) \$1.60.

james's Talks to Teachers on Psychology.

By William James, Professor in Harvard University, author of

"Principles of Psychology." xii + 301 pp. 12mo. \$1.50.

Jastrow's Chapters in Modern Psychology.

By Joseph Jastrow, Professor in the University of Wisconsin. [in preparation.] 
NEW BOOKS ON THE LIVING ISSUES BY LIVING MEN AND WOMEN

\section{The Home University Library}

Cloth Bound 50c per volume net; by mail 56c.

Points about THE HOME UNIVERSITY LIBRARY

Every volume is absolutely new, and specially written for the Library. There are no reprints.

Every volume is sold separately. Each has illustrations where needed, and contains a Bibliography as an aid to further study.

Every volume is written by a recognized authority on its subject, and the Library is published under the direction of four eminent Anglo-Saxon scholars-Gilbert MurRay, of Oxford; H. A. L. Fisher, of Oxford; J. ARthur Thomson, of Aberdeen; and Prof. W. T. Brewster, of Columbia.

Every subject is of living and permanent interest. These books tell whatever is most important and interesting about their subjects.

Each volume is complete and independent; but the series has been carefully planned as a whole to form a comprehensive library of modern knowledge covering the chief subjects in History and Geography, Literature and Art, Science, Social Science, Philosophy, and Religion. An order for any volume will insure receiving announcements of future issues. SOME COMMENTS ON THE SERIES AS A WHOLE: "Excellent."-The Outlook. "Exceedingly worth while."-The Nation. "The excellence of these books." -The Dial.

"So large a proportion with marked individuality."-New York Sun.

VOLUMES ON PHILOSOPHY AND RELIGION NOW READY

\section{Problems of Philosophy}

By BERTRANd RUSSEll.

\section{Ethics}

BY G. E. MOORE.

\section{Buddhism:}

A Study of the Buddhist Norm.

By Mrs. Rhys Davids, M.A.

\section{English Sects}

A History of Nonconformity.

By W. B. Selbie.

\section{Missions}

By Mrs. Creighton.

The Literature of the Old Teatament

By GEorge F. MOORE.

Comparative Religion

By J. Estlin Carpenter.

The Making of the New Testament

By B. W. BACON.

HENRY HOLT AND COM PANY 

RETURN CIRCULATION DEPARTMENT 15931 IO $\longrightarrow 202$ Main Library

\begin{tabular}{l|l|l}
$\begin{array}{c}\text { LOAN PERIOD } \\
\text { HOME USE }\end{array}$ & 2 & 3 \\
\hline 4 & 5 & 6 \\
\hline
\end{tabular}

ALL BOOKS MAY BE RECALLED AFTER 7 DAYS

Renewals and Recharges may be made 4 days prior to the due date.

Books may be Renewed by calling 642-3405.

\section{DUE AS STAMPED BELOW}

\begin{tabular}{l|l|l}
\hline MAY 26 1992 & & \\
\hline AUTO. DISC. & & \\
\hline MAY 27 1952 & & \\
\hline CIRCULATION & & \\
\hline APR1 3 1993 & & \\
\hline TODSCCIRC MUY & 0493 & \\
\hline & & \\
\hline
\end{tabular}

FORM NO. DD6

UNIVERSITY OF CALIFORNIA, BERKELEY BERKELEY, CA 94720 
U.C. BERKELEY LIBRARIES

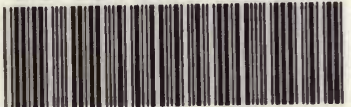

c038617671
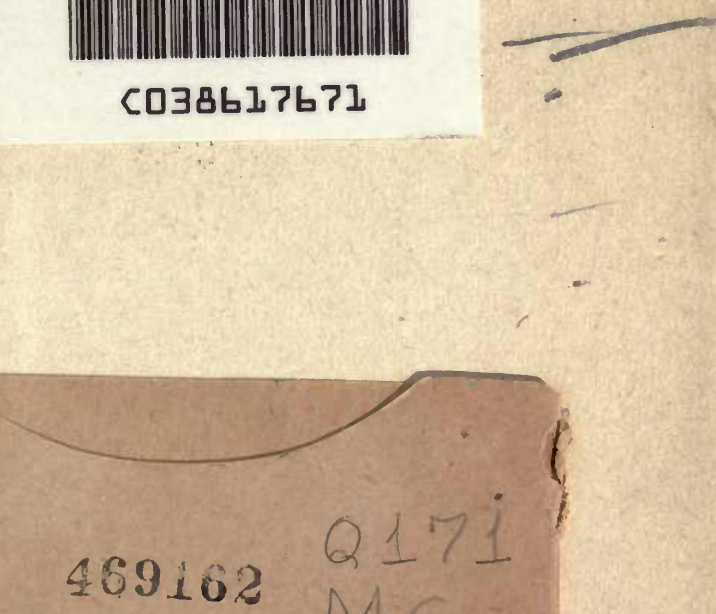

Q171

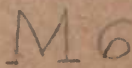

UNIVERSITY OF CALIFORNIA LIBRARY 
